\title{
Self-Assembling [n.n]Paracyclophanes: A Structure-Property Relationship Study
}

Will R. Henderson, Yu Zhu, Danielle E. Fagnani, Guancen Liu, Khalil A. Abboud, Ronald K. Castellano*

\section{Table of Contents}

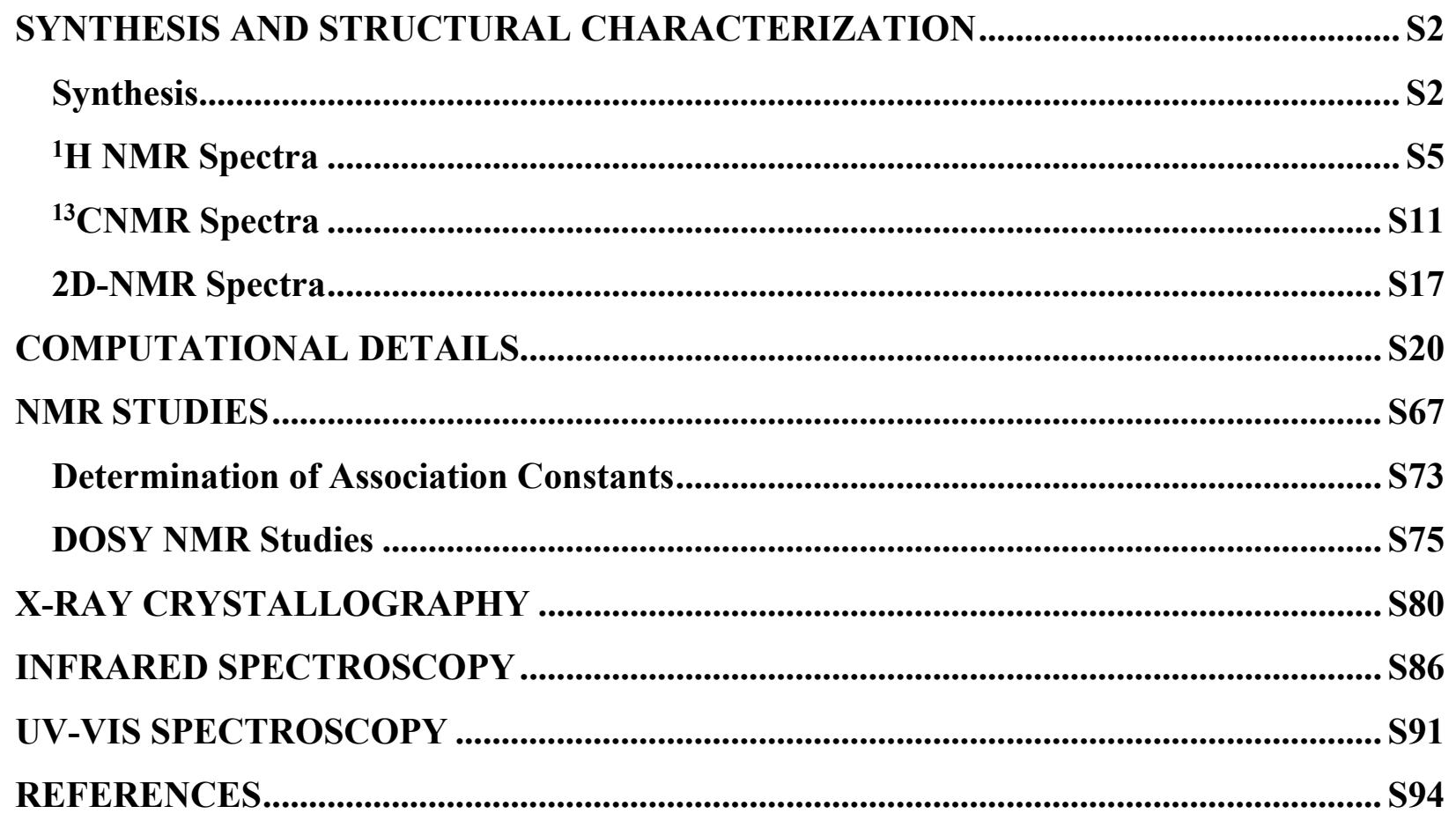




\section{SYNTHESIS AND STRUCTURAL CHARACTERIZATION}

\section{Synthesis}

Method A: [3.3]pCpTA ( \pm -1 was synthesized from [3.3]pCp, which was prepared according to literature procedures. ${ }^{1-3}$ The synthesis is shown in Scheme S1. $\alpha, \alpha$.-Dibromoxylene (4) is condensed with two equivalent of toluenesulfonylmethyl isocyanide (TosMIC) to give TosMIC adduct $\mathbf{1 0}^{2}$ to form cyclic tosmic adduct, which upon hydrolysis the hydrolysis yields [3.3]pCpdiketone 11. ${ }^{1}$ Wolff-Kishner-Huang reduction of 11 yields [3.3]pCp. ${ }^{3}$ [3.3]pCp is tetrabrominated to yield $( \pm)-7$, a novel compound. Tetraacid $( \pm)-8$, also a novel compound, is prepared through lithium-halogen exchange of $( \pm)-7$ with $t$-BuLi in THF, followed by quenching with $\mathrm{CO}_{2}$ in a modified protocol of preparation of [2.2]pCp-tetraacid in our previous report. ${ }^{4}$ Compound $( \pm)-8$ was converted to the tetraamide $( \pm)-1$ through the acid chloride intermediate.

Method B: [3.3]pCpTA $( \pm)-1$ was also prepared from 5-bromo-[3.3]pCp $( \pm$ )-6 (shown in Scheme S2) by fully brominating to $( \pm)-7$. The subsequent conversions are the same as above. Synthesis of $( \pm)-6$ proceeded through a modification of a literature procedure by macrocyclization of 4 and $5,{ }^{5}$ followed by Wolff-Kishner-Huang reduction of [3.3]pCpBr-diketone ( \pm )-12.

Comparator [3.3]pCp-5-carboxamide $( \pm)$-3 was synthesized from $( \pm)-6$ by carboxylation to the corresponding acid $( \pm)-9$ through lithium-halogen exchange of $( \pm)-6$ with $t$-BuLi in THF, followed by quenching with $\mathrm{CO}_{2}$ and finally [3.3]pCp-5-carboxamide $( \pm)-3$ via the above discussed method. 


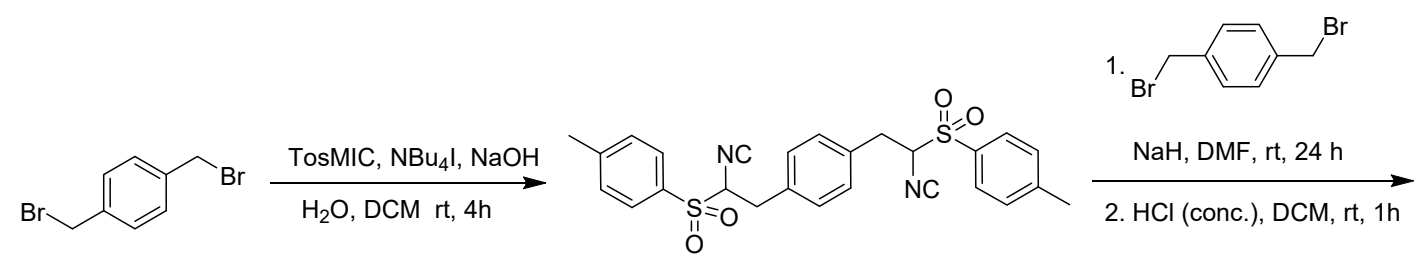

4

$64 \%$

10

$50 \%$<smiles></smiles>

1. $\mathrm{N}_{2} \mathrm{H}_{4} \cdot \mathrm{H}_{2} \mathrm{O}$, $\mathrm{N}_{2} \mathrm{H}_{4} \cdot 2 \mathrm{HCl}$, diethylene glycol $130{ }^{\circ} \mathrm{C}, 6 \mathrm{~h}$

2. $\mathrm{KOH}$

$200{ }^{\circ} \mathrm{C}, 5 \mathrm{~h}$<smiles>C1=CC2=C3CCCC1=CC=C3CCC2</smiles><smiles>O=C(O)CC(CBr)c1ccccc1Br</smiles><smiles>Brc1cc(Br)c(CCCC2CCCc3c(Br)ccc(Br)c32)cc1Br</smiles>

1. $t$-BuLi (10 equiv) $-78{ }^{\circ} \mathrm{C}$ to rt, $2 \mathrm{~h}$

$68 \%$

[3.3]pCp $\quad 52 \%$

(士)-7

$84 \%$

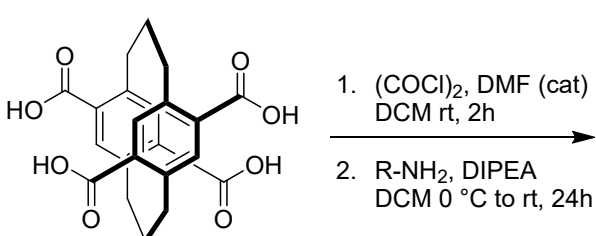

(士)-8

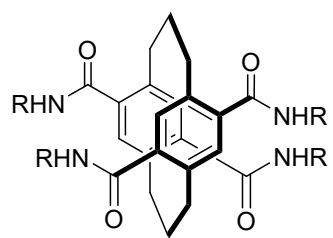

( $)-1$ ([3.3]pCpTA)

Scheme S1. Synthesis of self-assembling [3.3]pCp-5,8,14,17-tetracarboxamide ( \pm )-1 by method A. 


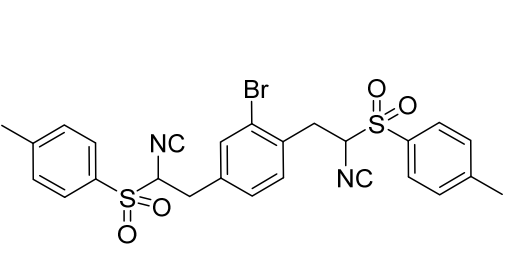

5

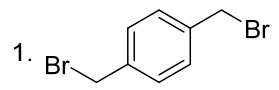

$\mathrm{NBu}_{4}, \mathrm{NaOH}$

$\mathrm{H}_{2} \mathrm{O}, \mathrm{DCM}$ (.008M), reflux $24 \mathrm{~h}$

2. $\mathrm{HCl}$ (conc.), DCM, rt, $1 \mathrm{~h}$

$48 \%$

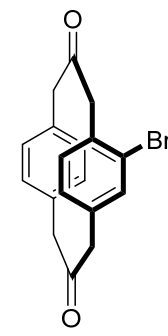

( \pm -12

\section{$\mathrm{N}_{2} \mathrm{H}_{4} \cdot \mathrm{H}_{2} \mathrm{O}$ then $\mathrm{KOH}$ \\ diethylene glycol \\ $130^{\circ} \mathrm{C}-200^{\circ} \mathrm{C}, 5 \mathrm{~h}$}

$86 \%$


$( \pm)-6$<smiles></smiles>

( \pm -9
1. $(\mathrm{COCl})_{2}, \mathrm{DMF}$ (cat)

2. $\mathrm{R}-\mathrm{NH}_{2}$, DIPEA $\mathrm{DCM} 0^{\circ} \mathrm{C}$ to rt, $24 \mathrm{~h}$

$34 \%$

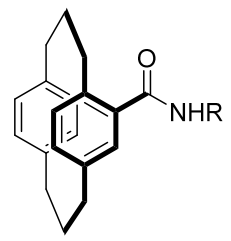

$( \pm)-3 \mathrm{R}=\mathrm{C}_{6} \mathrm{H}_{13}$

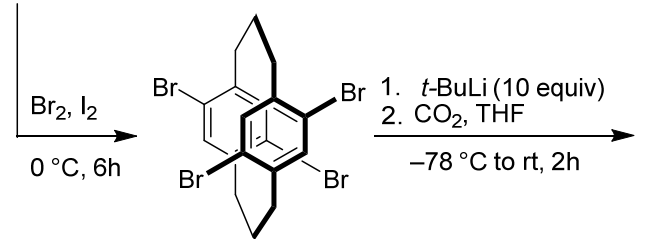

$63 \%$

$( \pm)-7$

$87 \%$

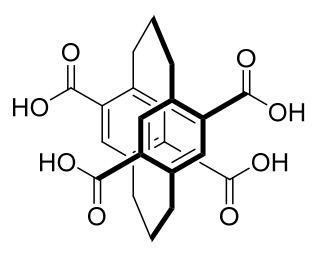

(士)-8
1. $(\mathrm{COCl})_{2}, \mathrm{DMF}$ (cat) DCM rt, $2 \mathrm{~h}$

2. $\mathrm{R}-\mathrm{NH}_{2}$, DIPEA $\mathrm{DCM} 0^{\circ} \mathrm{C}$ to $\mathrm{rt}, 24 \mathrm{~h}$

$22-41 \%$

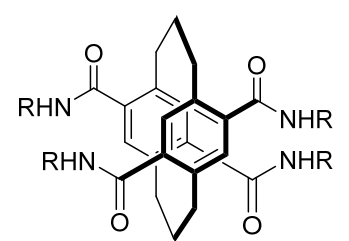

( \pm -1 ([3.3]pCpTA) (士)-1a, $\mathrm{R}=\mathrm{C}_{6} \mathrm{H}_{13}$

(士)-1b, $\mathrm{R}=\mathrm{C}_{3} \mathrm{H}_{7}$

(士)-1c, $\mathrm{R}=\mathrm{Bn}$

(士)-1d, $\mathrm{R}=\mathrm{Ph}$

(士)-1e, $\mathrm{R}=\mathrm{C}_{2} \mathrm{H}_{5}$

Scheme S2. Synthesis of comparator [3.3]pCp-5-carboxamide ( \pm )-3 and [3.3]pCp-5,8,14,17-tetracarboxamide ( \pm )-1 by method B. 


\section{${ }^{1} \mathrm{H}$ NMR Spectra}
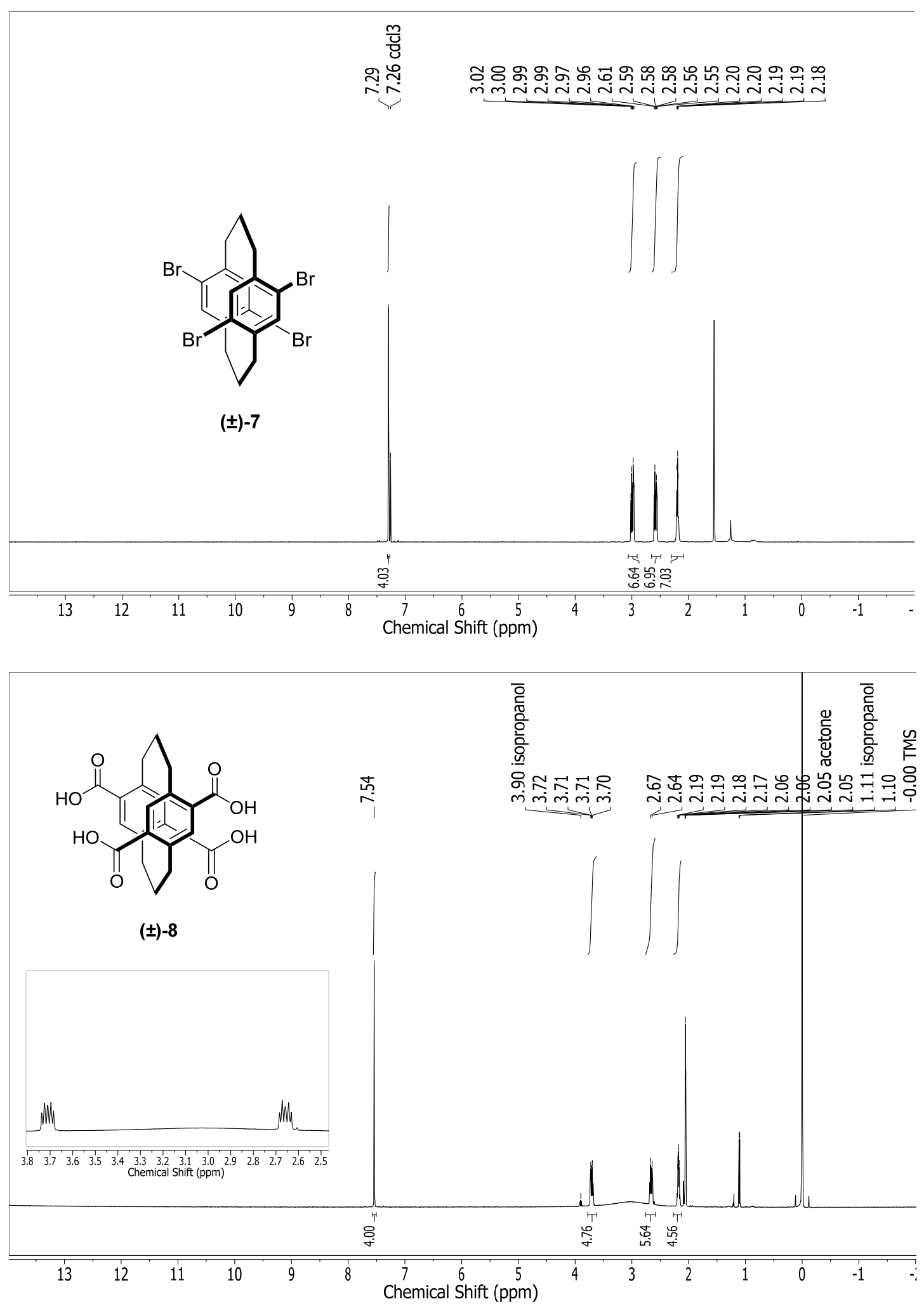

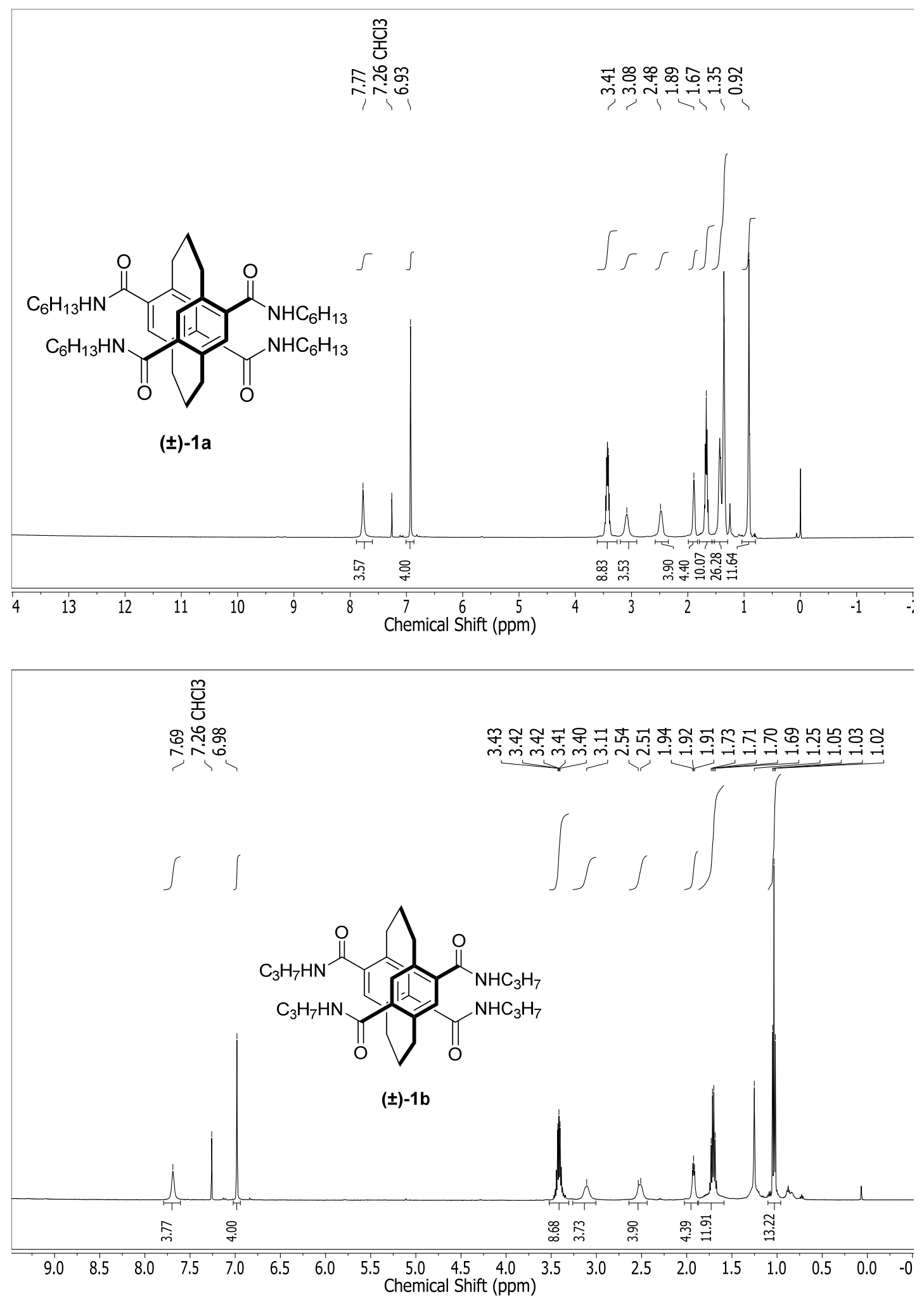

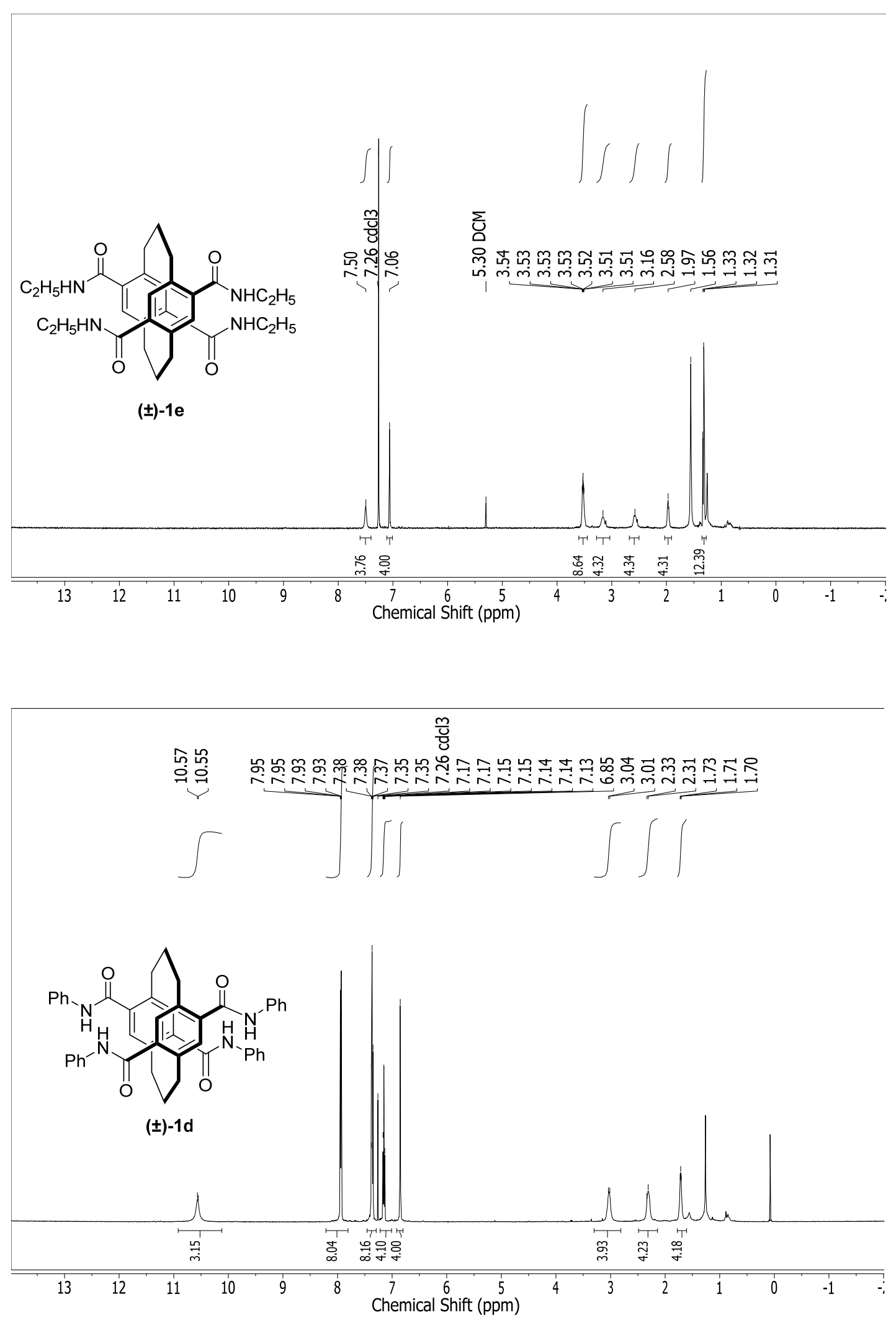

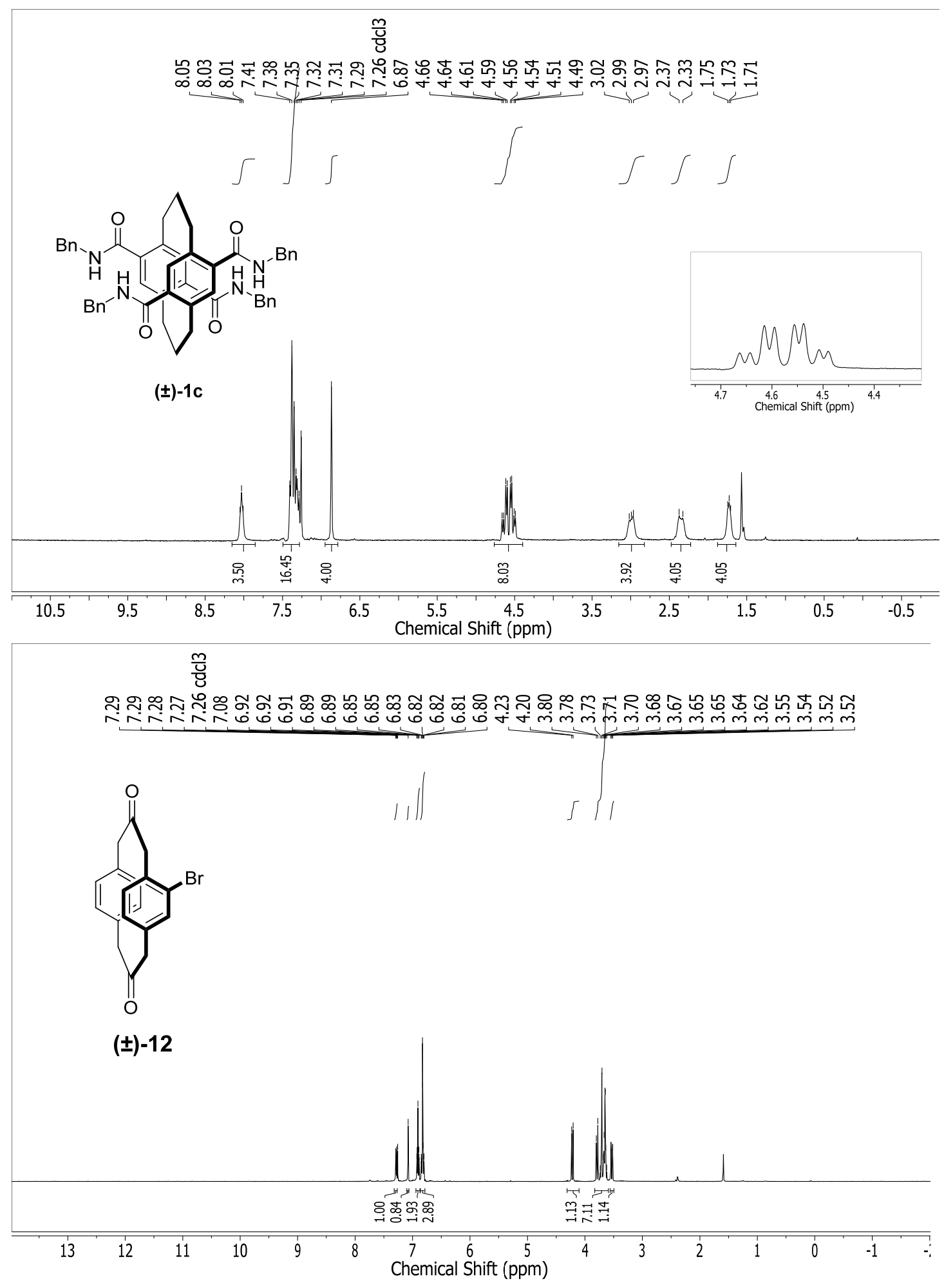

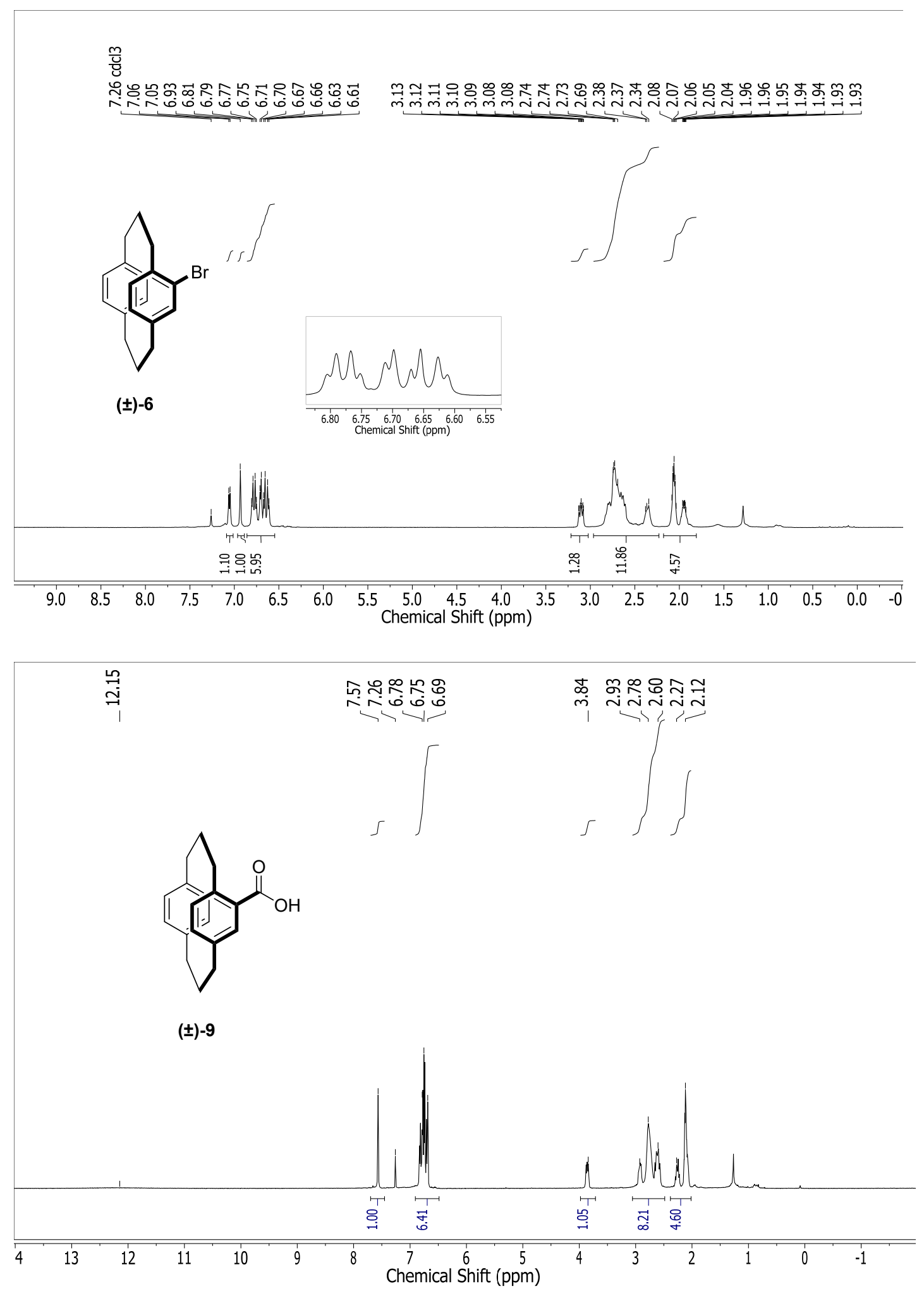


\section{鲂}

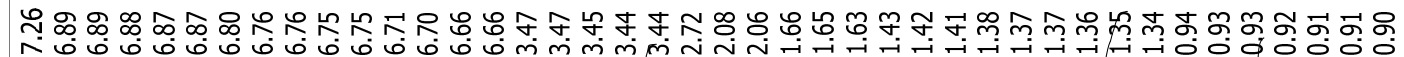
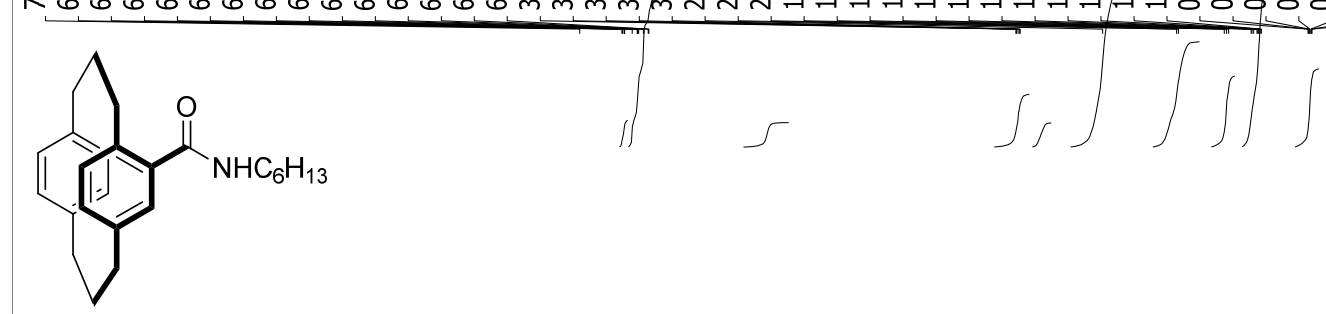

(士)-3
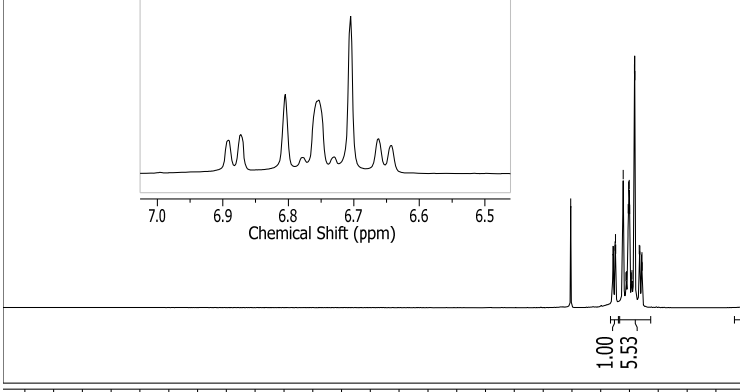

$\begin{array}{llllllll}11.5 & 10.5 & 9.5 & 8.5 & 7.5 & 6.5\end{array}$ Chemical Shift (ppm)

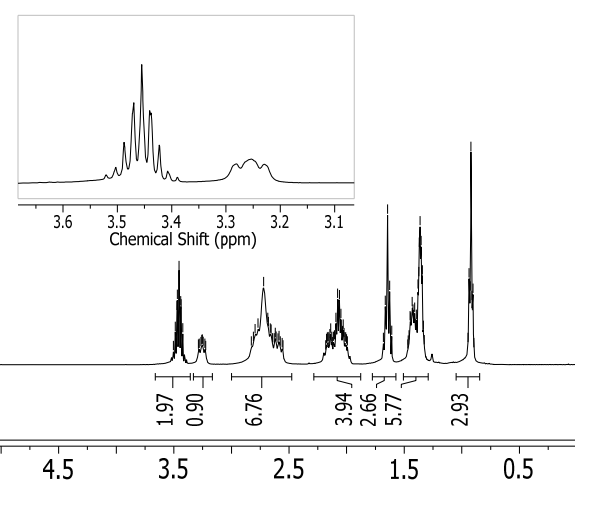



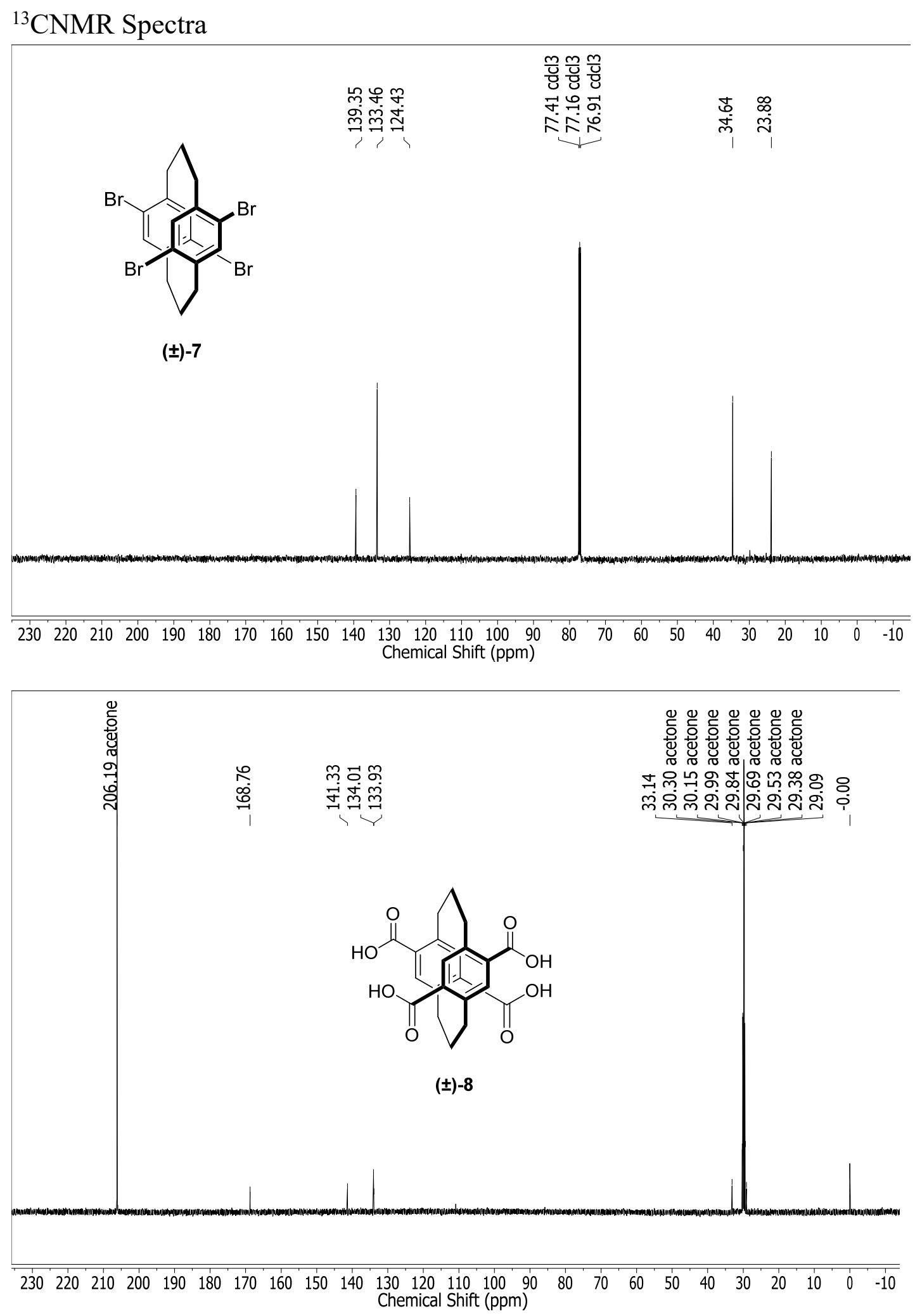

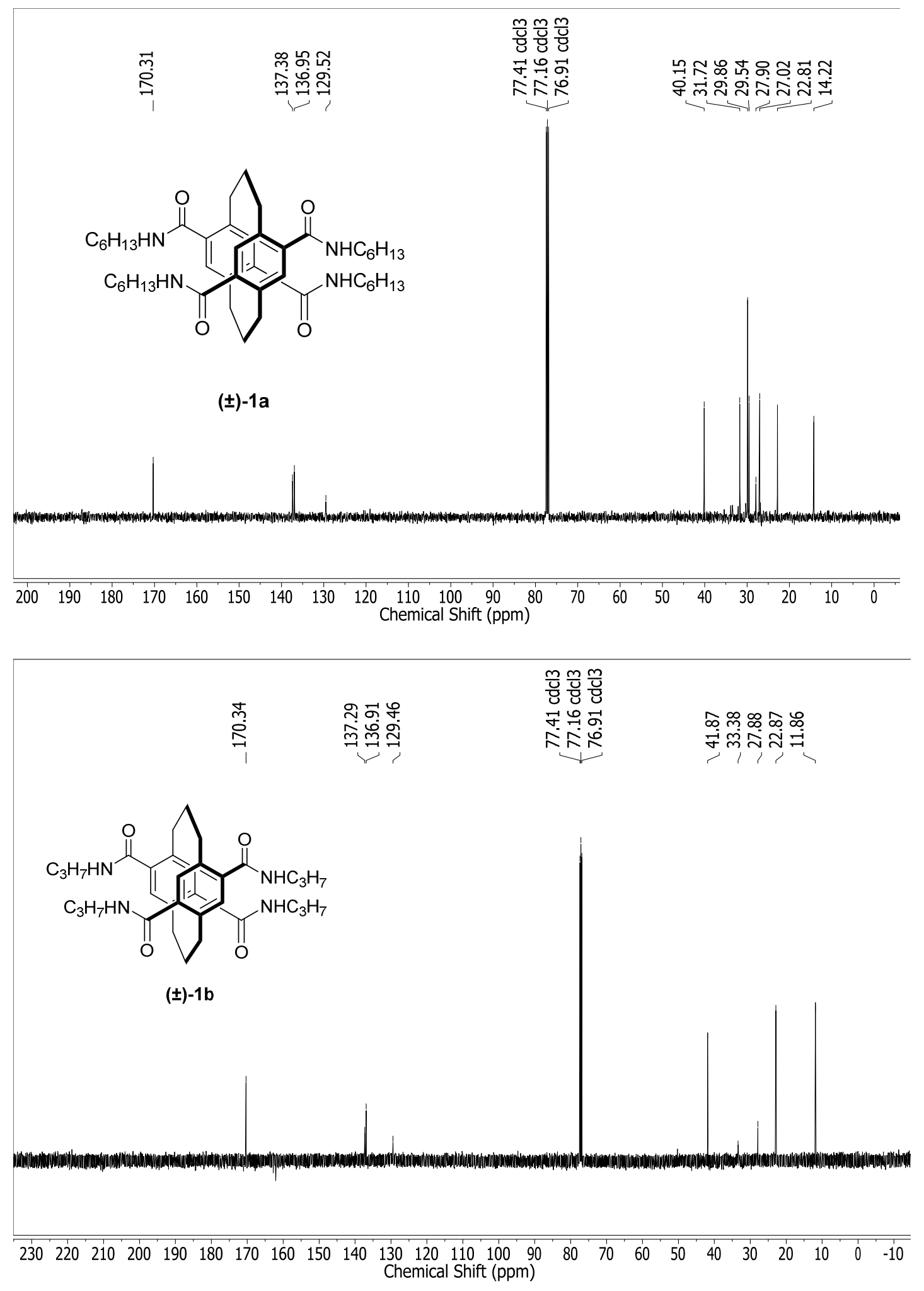

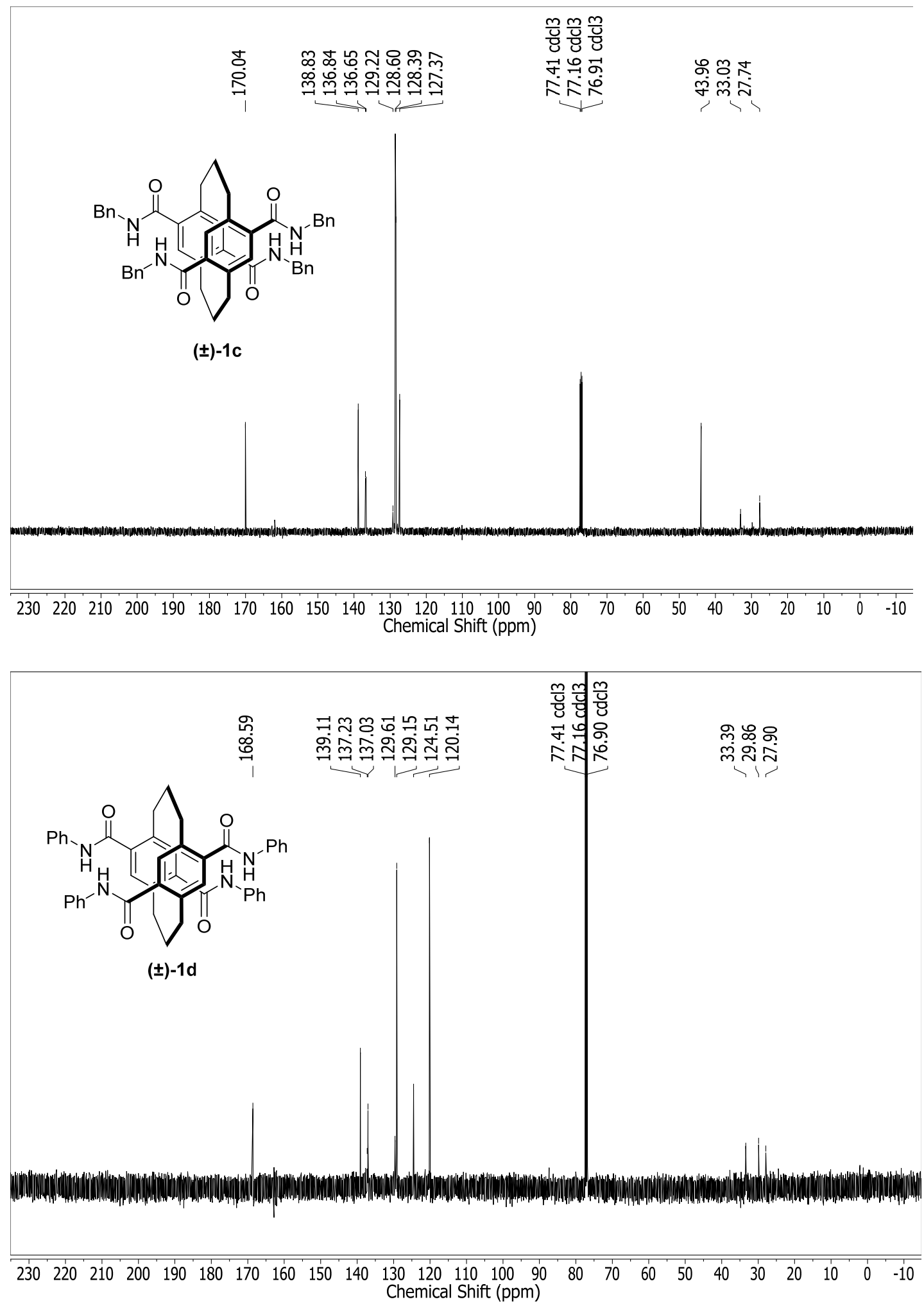

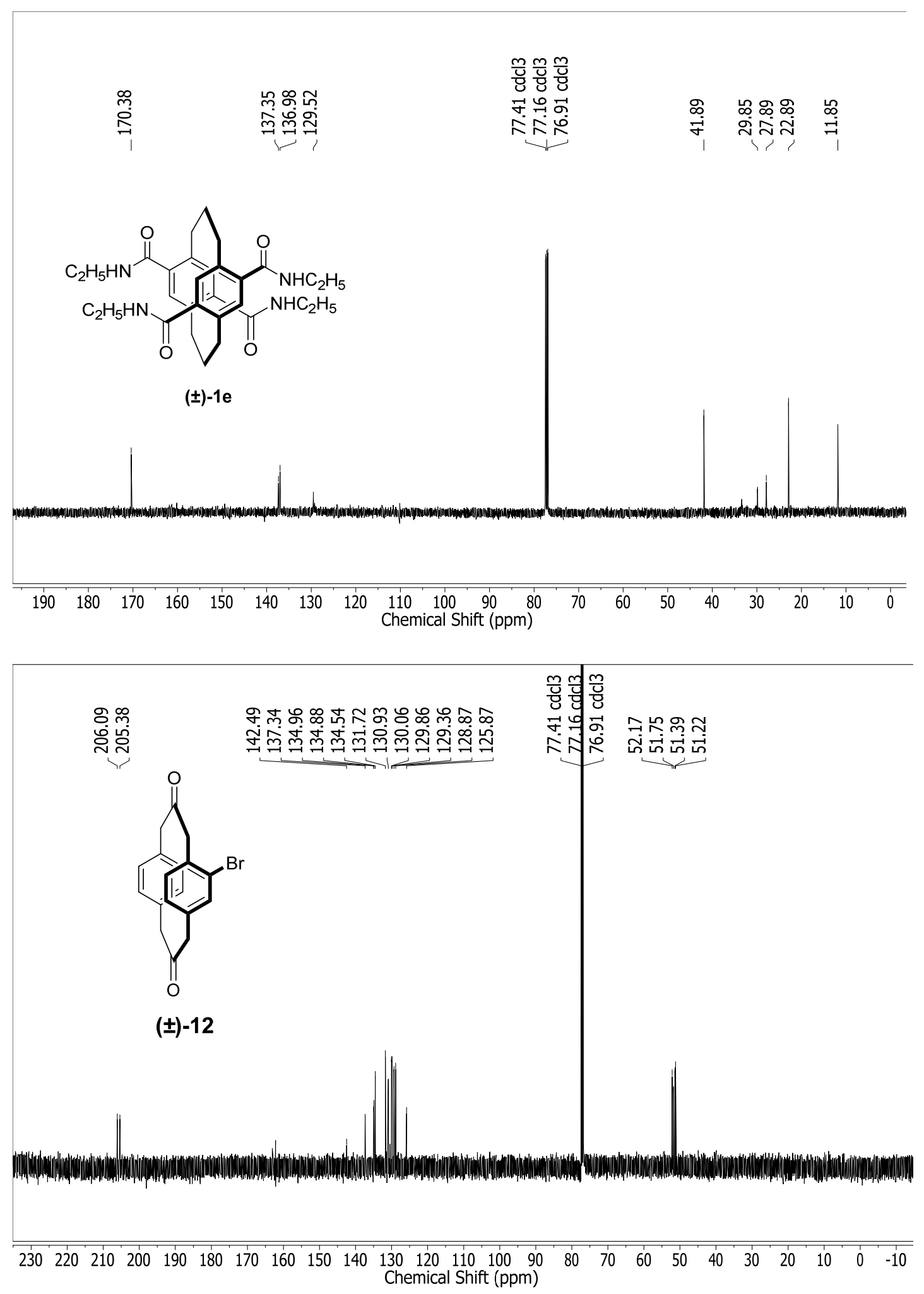

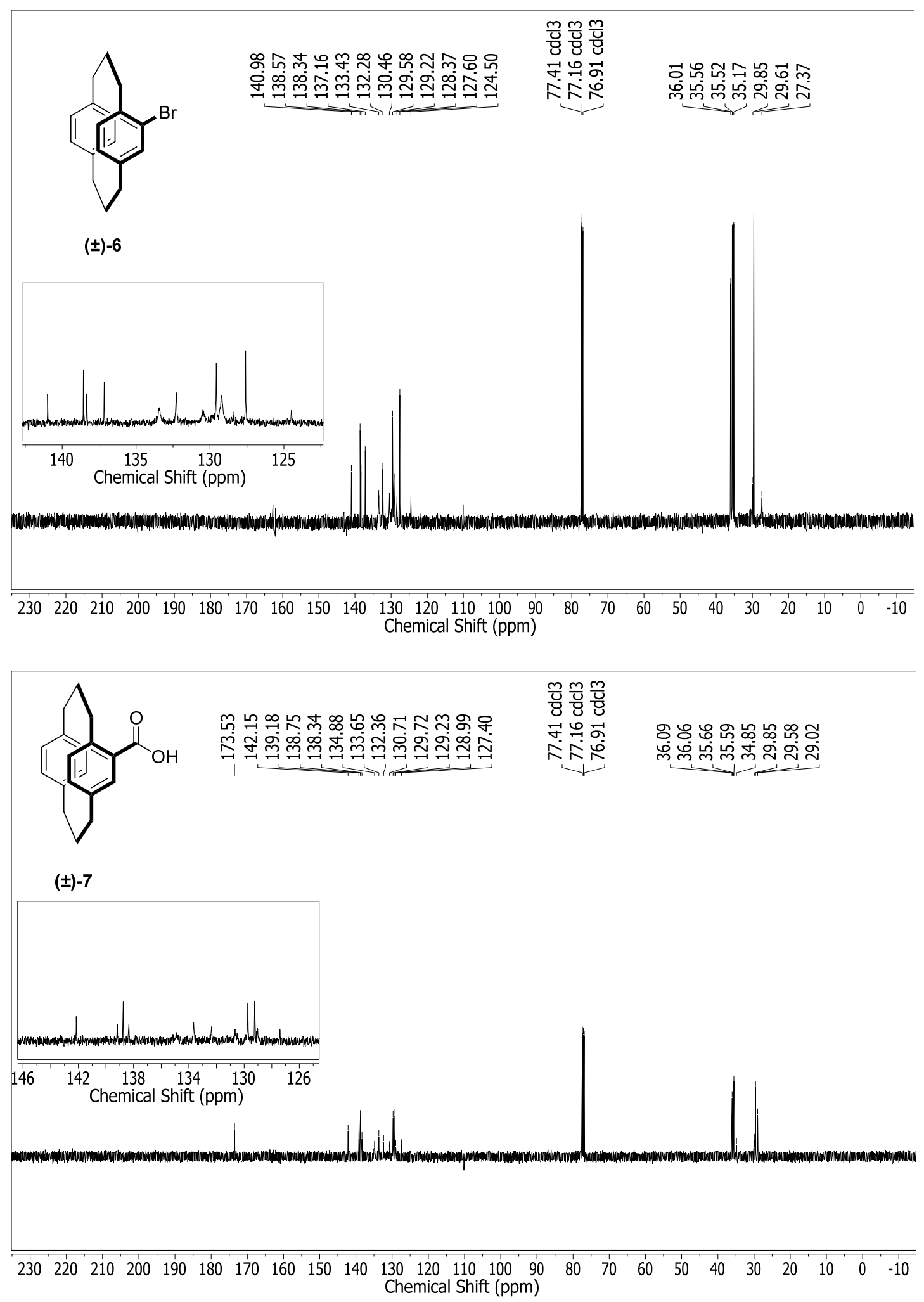


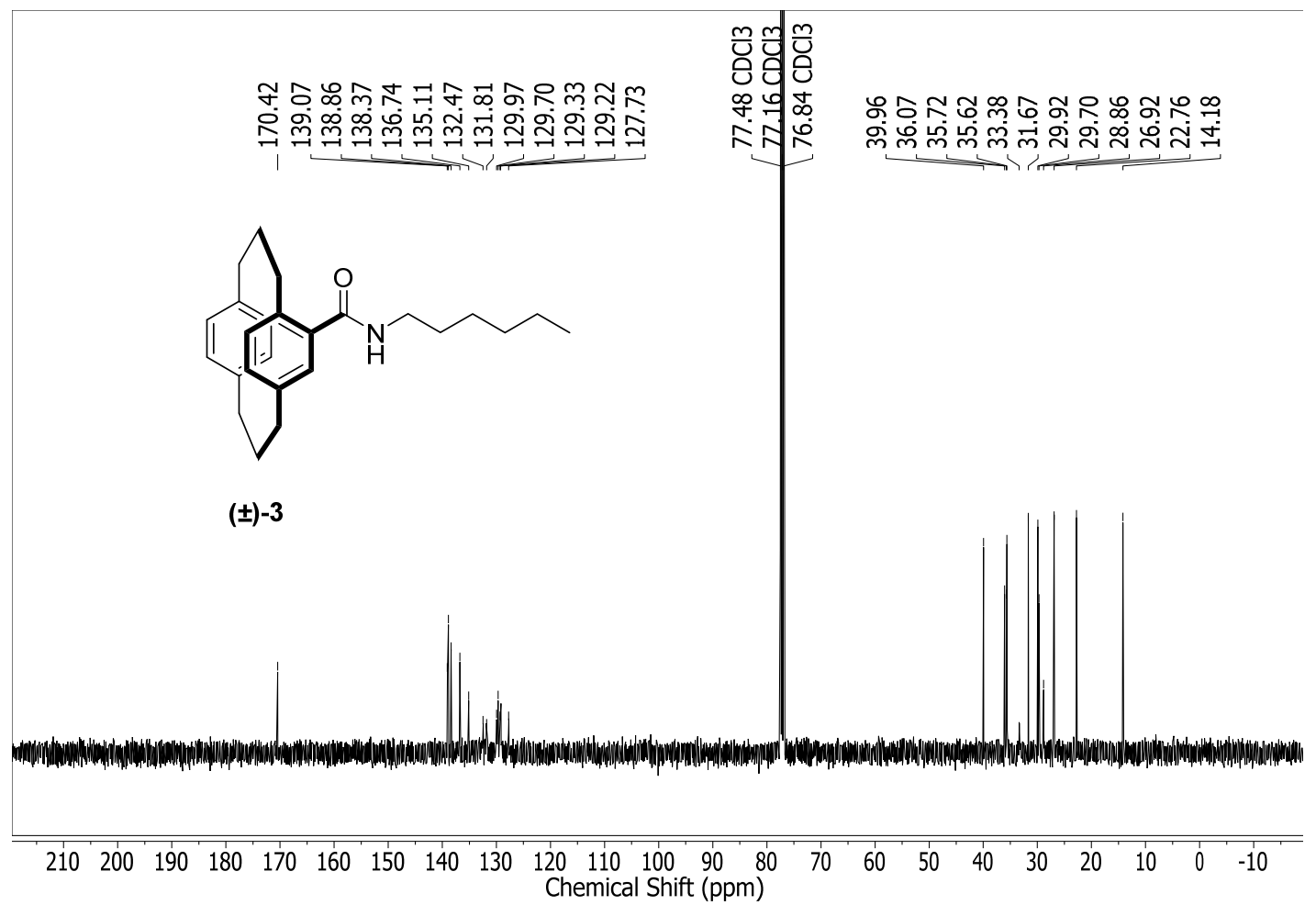



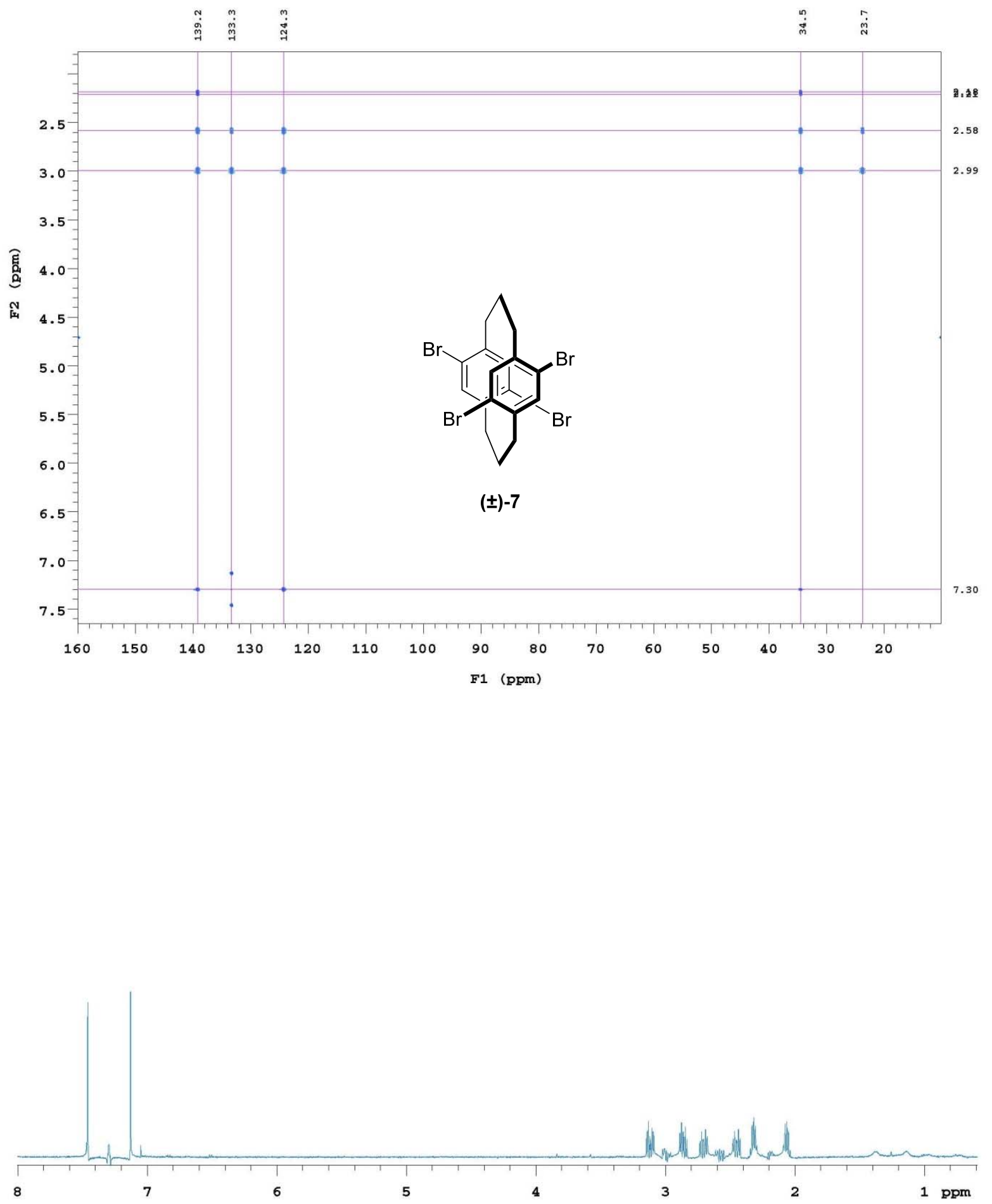

Figure S1. gHMBC (above) and gHSQC (below) spectrum of ( \pm )-7. 

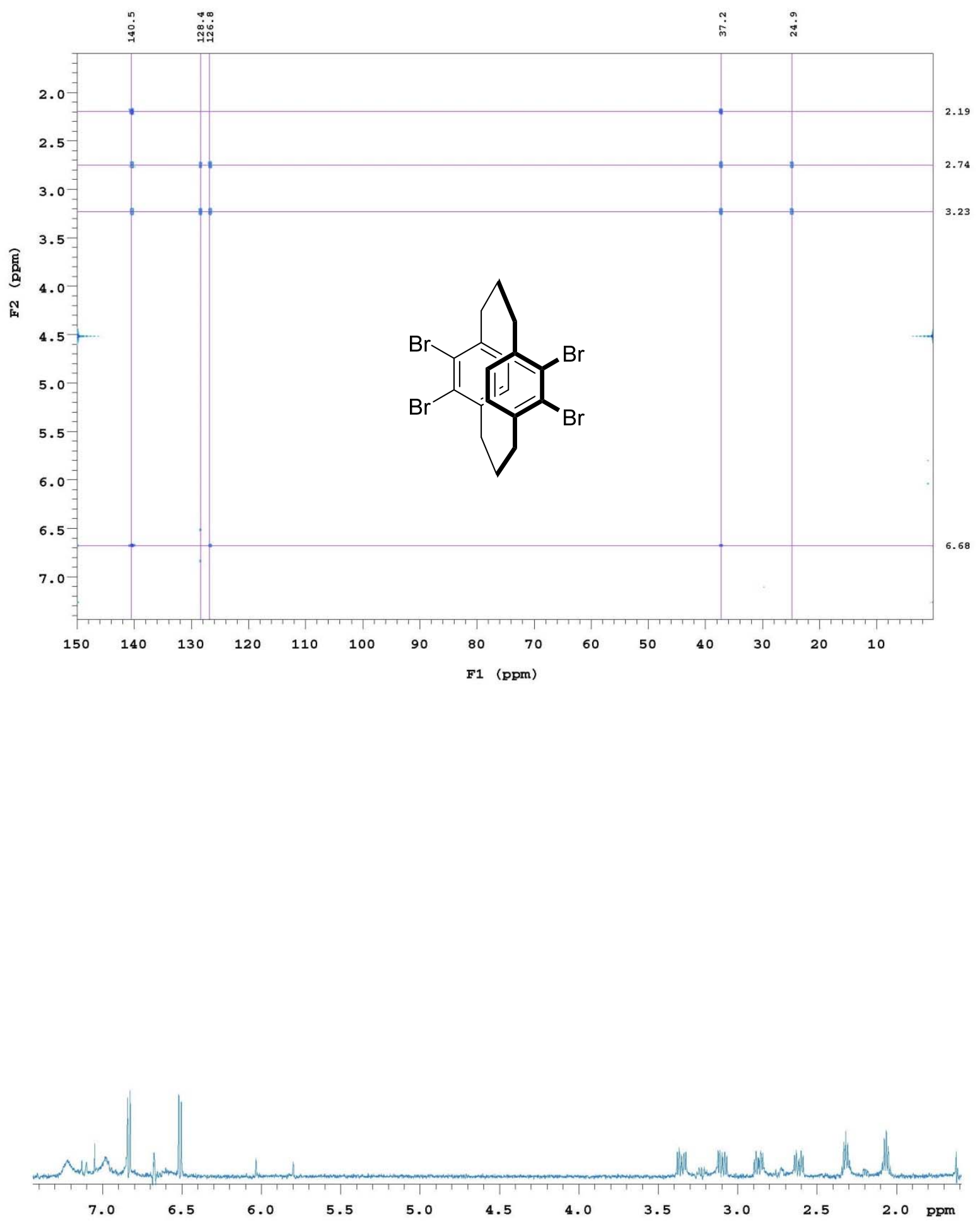

Figure S2. gHMBC (above) and gHSQC (below) spectrum of ( \pm )-5,6,14,15-tetrabromo[3.3]paracyclophane. 


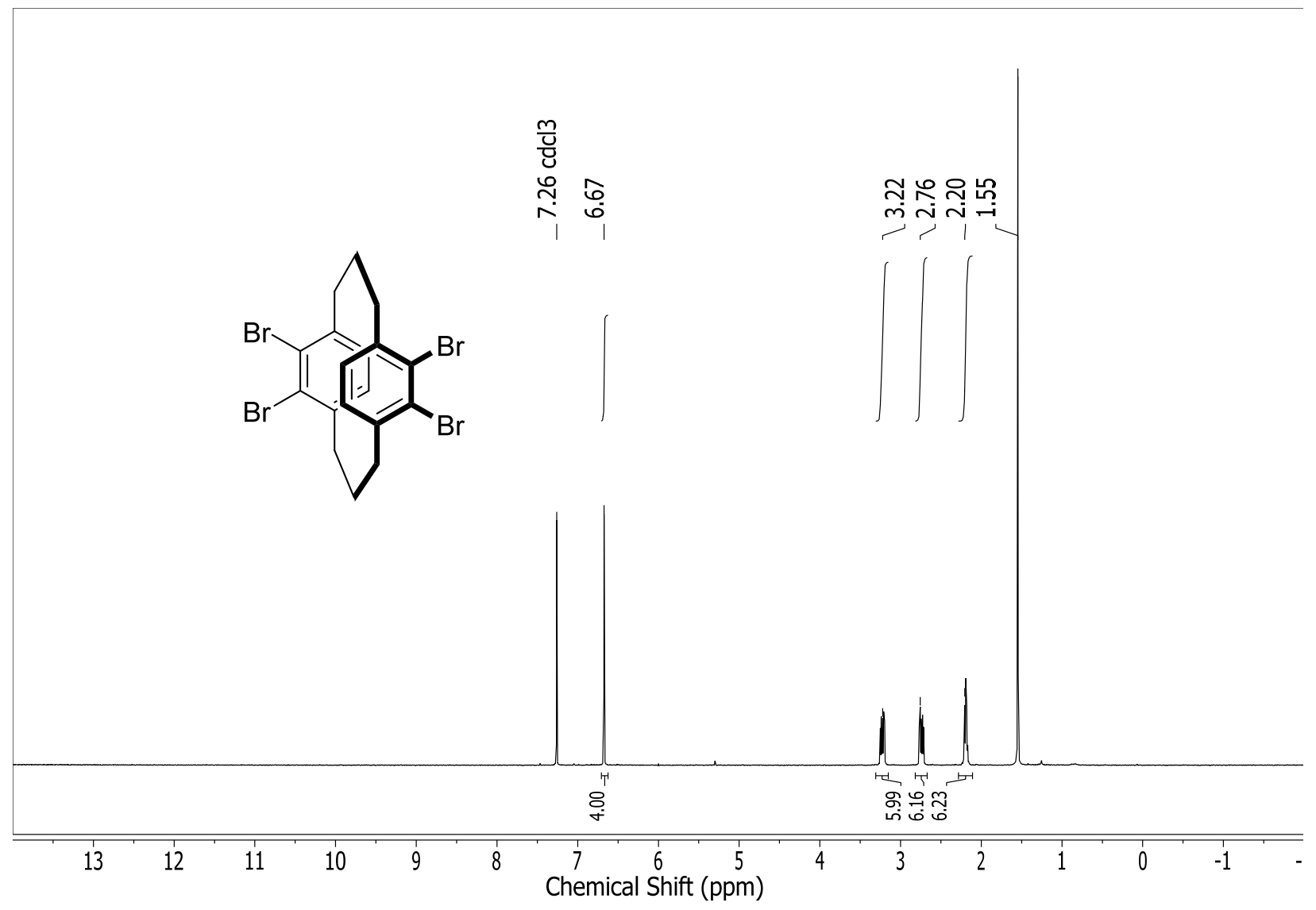

Figure S3. ${ }^{1} \mathrm{H}$ NMR spectrum of ( \pm )-5,6,14,15-tetrabromo[3.3]paracyclophane. 


\section{COMPUTATIONAL DETAILS}

\section{General Information}

An X-ray crystal structure, CSD reference code PCYCPH, was used to establish the starting structure of [3.3]paracyclophane in the chair conformation. The starting boat conformation was established using coordinates from published calculations. ${ }^{1}$

Upon addition of a single $N$-methylamido group (to $\mathrm{C}_{5}$ of the chair or boat conformation of [3.3]pCp), the central bridge carbon $\left(\mathrm{C}_{2}\right)$ can point towards $(s y n)$ or away (anti) from the amide group (note the terminology is separate from the one used to describe relative amide bond directionality in cases where there are two or more amides). Four classes of conformations result (boat_anti,boat_syn, chair_anti, and chair_syn). A conformational search was executed by fixing all of the atoms of the paracyclophane backbone and evaluating the energy change upon rotation of the $\mathrm{O}=\mathrm{C}-\mathrm{C}_{5}-\mathrm{C}_{6}$ dihedral angle $\left(0^{\circ}\right.$ to $360^{\circ}$ by every $\left.1^{\circ}\right)$ in molecular mechanics method using OPLS3 force field in Maestro ${ }^{2}$ (Version 11.1.012) as performed by MacroModel $^{3}$ (Version 11.5.012). Four local minima were found among the 360 anti conformers in both boat (boat_anti) and chair (chair_anti) backbone conformations, and two local minima were found among the syn conformers in both backbone conformations (boat_syn and chair_syn). The 12 low-energy conformers were then subjected to DFT calculations as described below.

The starting geometries of the pseudo-ortho bisamides were obtained by addition of two $N$-methylamido group to $\mathrm{C}_{5}$ and $\mathrm{C}_{14}$ of the parent unsubstituted [3.3]pCp backbones, and manually positioning the amide groups (through adjusting the $\mathrm{O}=\mathrm{C}-\mathrm{C}_{5}-\mathrm{C}_{6}$ and $\mathrm{O}=\mathrm{C}-\mathrm{C}_{14}-\mathrm{C}_{15}$ dihedral angles) in a hydrogen bond forming conformation where the $\mathrm{N}-\mathrm{H}^{\cdots} \mathrm{O}$ hydrogen bond have good linearity.

The starting geometries of the pseudo-para bisamides were obtained by addition of two $N$-methylamido group to $\mathrm{C}_{5}$ and $\mathrm{C}_{18}$ of the parent unsubstituted [3.3]pCp backbones, and their $\mathrm{O}=\mathrm{C}-\mathrm{C}_{5}-\mathrm{C}_{6}$ and $\mathrm{O}=\mathrm{C}-\mathrm{C}_{18}-\mathrm{C}_{17}$ dihedral angles were set with the two following rules: (1) The pseudo-para bisamides have the same amide bond directions as the pseudo-ortho bisamides, which means the $\mathrm{O}=\mathrm{C}-\mathrm{C}_{5}-\mathrm{C}_{6}$ and $\mathrm{O}=\mathrm{C}-\mathrm{C}_{18}-\mathrm{C}_{17}$ dihedral angles are similar to the $\mathrm{O}=\mathrm{C}-\mathrm{C}_{5}-\mathrm{C}_{6}$ and $\mathrm{O}=\mathrm{C}-\mathrm{C}_{14}-\mathrm{C}_{15}$ dihedral angles of their corresponding pseudo-ortho bisamides (with the same $\mathrm{pCp}$ chair/boat conformation). (2) The exact value are set according to the $\mathrm{O}=\mathrm{C}-\mathrm{C}_{5}-\mathrm{C}_{6}$ dihedral angle of the corresponding geometry optimized monoamides (with the same backbone conformation and the same relative amide group-adjacent bridge conformation). For example, the starting $\mathrm{O}=\mathrm{C}-\mathrm{C}_{5}-\mathrm{C}_{6}$ dihedral angle of pseudopara_l should be similar that of pseudoortho_l $\left(-142.6^{\circ}\right.$, so the two amide group are in the same direction) and was set at $-140.3^{\circ}$ (as the $\mathrm{O}=\mathrm{C}-\mathrm{C}_{5}-\mathrm{C}_{6}$ dihedral angle of boat_anti_3). And the starting $\mathrm{O}=\mathrm{C}-\mathrm{C}_{18}-\mathrm{C}_{17}$ dihedral angle should be similar to the $\mathrm{O}=\mathrm{C}-\mathrm{C}_{14}-\mathrm{C}_{15}$ dihedral angle of pseudoortho_1 $\left(47.6^{\circ}\right.$, but $-47.6^{\circ}$ for $\mathrm{C}_{18}$ position) and was set at $-44.4^{\circ}$ as boat_syn_1. Four of the monoamides (boat_anti_2, boat_anti_4, chair_anti_2, and chair_anti_4) were not referenced because such amide conformations do not promote intramolecular hydrogen 
bond formation in pseudo-ortho bisamides. The optimized energies were first compared between model compounds with the same backbone (i.e. pseudopara_l with pseudoortho_l). Additionally, comparison between model compounds with the same (syn or anti) amide group-adjacent bridge conformation (pseudoortho_1 with pseudopara_3, pseudoortho_2 with pseudopara_4, pseudoortho_3 with pseudopara_1, and pseudoortho_4 with pseudopara_2) for both amide groups provides more insight to the energy differences because the interaction between the amides groups and the ortho- benzylic hydrogen are the same. For example, pseudoortho_l have both $\mathrm{C}_{5}$ and $\mathrm{C}_{14}$ amide groups in anti conformation and the corresponding pseudopara_3 have both $\mathrm{C}_{5}$ and $\mathrm{C}_{18}$ amide groups in anti conformation.

The starting geometries of the tetraamides were obtained by addition of four $\mathrm{N}$-methylamido group to $\mathrm{C}_{5}, \mathrm{C}_{8}, \mathrm{C}_{14}$, and $\mathrm{C}_{17}$ of the parent unsubstituted [3.3]pCp backbones and setting their dihedral angles of amide groups on each side according to the dihedral angles of the corresponding pseudopara bisamides (geometry optimized) with the same amide group-ortho trimethylene bridge conformation. For example, the $\mathrm{O}=\mathrm{C}-\mathrm{C}_{5}-\mathrm{C}_{6}$ (anti) and $\mathrm{O}=\mathrm{C}-\mathrm{C}_{14}-\mathrm{C}_{15}$ (syn) dihedral angles of anti_chair were set as those of pseudoortho_3, while the $\mathrm{O}=\mathrm{C}-\mathrm{C}_{17}-\mathrm{C}_{18}$ (syn) and $\mathrm{O}=\mathrm{C}-\mathrm{C}_{8}-\mathrm{C}_{9}$ (anti) dihedral angles were set as the $\mathrm{O}=\mathrm{C}-\mathrm{C}_{5}-\mathrm{C}_{6}\left(\right.$ syn) and $\mathrm{O}=\mathrm{C}-\mathrm{C}_{14}-\mathrm{C}_{15}$ (anti) dihedral angles of pseudoortho_2.

The starting geometries of dimers of tetraamide were obtained from manually positioning geometrically optimized tetraamide in hydrogen bonding forming position in Spartan Student version 5.0.2 for Macintosh.

The structural geometries of all monomers and dimers were optimized at the M06-2X/6-31+ $\mathrm{G}^{*}$ level of theory (as implemented in Gaussian 09). Frequency calculations were performed at the same computational level for all monomers, and no imaginary frequencies were found except one of the tetraamides (a negative frequency is found for tetraamide syn_boat and the frequency is associate with the conformation of a methyl group). Dihedral angles were measured in GaussianView.

a)

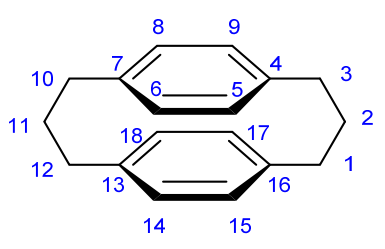

b)

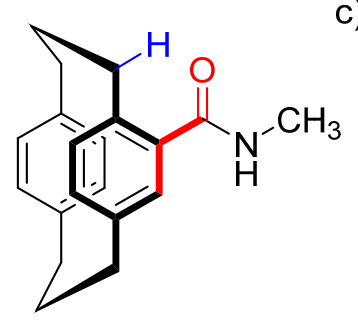

c)

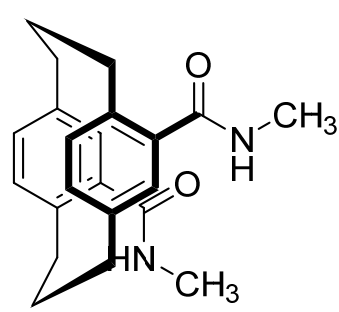

d)

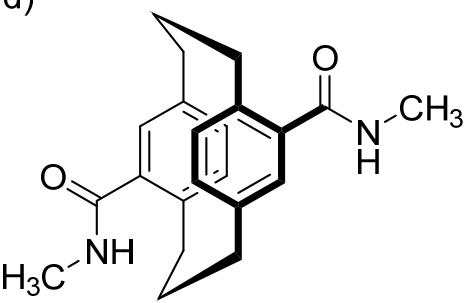

Figure S4. [3.3]paracyclophane and model compounds. a) numbering system, b) $\left(R_{\mathrm{p}}\right)-[3.3] \mathrm{pCp}-5-$ monocarboxamide (dihedral angle $\mathrm{O}=\mathrm{C}-\mathrm{C}_{5}-\mathrm{C}_{6}$ colored in red, ortho-benzylic hydrogen in blue), c) "pseudo-para" [3.3]pCp-5,14-dicarboxamide, and d) "pseudo-para" [3.3]pCp-5,18-dicarboxamide. 
Table S1. Summary of geometry optimization results. Dihedral angles were measured between carbonyl groups and adjacent ring carbons (for example: $\mathrm{O}=\mathrm{C}-\mathrm{C}_{5}-\mathrm{C}_{6}$ on the mono-substituted derivative) and the total energies were normalized for each group of structural isomers.

\begin{tabular}{|c|c|c|c|c|c|c|c|}
\hline \multicolumn{2}{|c|}{ [3.3]pCp derivatives } & $\begin{array}{l}\phi_{\text {dihedral }} \\
\mathrm{C}_{5}-\mathrm{CO} \\
\left({ }^{\circ}\right)\end{array}$ & $\begin{array}{l}\phi_{\text {dihedral }} \\
\mathrm{C}_{8}-\mathrm{CO} \\
\left({ }^{\circ}\right)\end{array}$ & $\begin{array}{l}\phi_{\text {dihedral }} \\
\mathrm{C}_{14}-\mathrm{CO} \\
\left(^{\circ}\right)\end{array}$ & $\begin{array}{l}\phi_{\text {dihedral }} \\
\mathrm{C}_{17}-\mathrm{CO} \\
\left({ }^{\circ}\right)\end{array}$ & $\begin{array}{l}\phi_{\text {dihedral }} \\
\mathrm{C}_{18}-\mathrm{CO} \\
\left(^{\circ}\right)\end{array}$ & $\begin{array}{l}\text { Normalized } \\
\text { Total Energy } \\
\text { (kcal/mol) }\end{array}$ \\
\hline $\begin{array}{l}\text { Mono } \\
\text { amide }\end{array}$ & $\begin{array}{l}\text { Boat_anti_1 } \\
\text { Boat_anti_2 } \\
\text { Boat_anti_3 } \\
\text { Boat_anti_4 } \\
\text { Boat_syn_1 } \\
\text { Boat_syn_2 } \\
\text { Chair_anti_1 } \\
\text { Chair_anti_2 } \\
\text { Chair_anti_3 } \\
\text { Chair_anti_4 } \\
\text { Chair_syn_1 } \\
\text { Chair_syn_2 }\end{array}$ & $\begin{array}{c}45.8 \\
129.8 \\
-140.3 \\
-43.0 \\
44.4 \\
-138.9 \\
48.2 \\
130.8 \\
-141.0 \\
-43.5 \\
44.6 \\
-137.5\end{array}$ & & & & & $\begin{array}{c}1.16 \\
0.18 \\
1.62 \\
4.41 \\
0.70 \\
0 \\
1.39 \\
0.02 \\
1.44 \\
4.40 \\
0.62 \\
0.30\end{array}$ \\
\hline $\begin{array}{l}\text { Di } \\
\text { amide }\end{array}$ & $\begin{array}{l}\text { Pseudoortho_1 } \\
\text { Pseudoortho_2 } \\
\text { Pseudoortho_3 } \\
\text { Pseudoortho_4 } \\
\text { Pseudopara_1 } \\
\text { Pseudopara_2 } \\
\text { Pseudopara_3 } \\
\text { Pseudopara_4 }\end{array}$ & $\begin{array}{l}-142.6 \\
-140.5 \\
-138.9 \\
-138.2 \\
-139.6 \\
-140.4 \\
-141.3 \\
-140.2 \\
\end{array}$ & & $\begin{array}{l}47.6 \\
50.6 \\
45.8 \\
47.1\end{array}$ & & $\begin{array}{l}-46.1 \\
-45.9 \\
-48.2 \\
-50.1\end{array}$ & $\begin{array}{c}4.32 \\
3.14 \\
3.82 \\
0 \\
8.16 \\
6.85 \\
8.95 \\
7.24 \\
\end{array}$ \\
\hline $\begin{array}{l}\text { Tetra } \\
\text { amide }\end{array}$ & $\begin{array}{l}\text { Anti_chair } \\
\text { Anti_boat } \\
\text { Syn_chair } \\
\text { Syn_boat }\end{array}$ & $\begin{array}{l}-132.4 \\
-135.5 \\
-140.7 \\
-137.2^{\circ}\end{array}$ & $\begin{array}{c}53.4 \\
51.5 \\
-140.7 \\
-141.8\end{array}$ & $\begin{array}{l}46.8 \\
49.3 \\
53.3 \\
46.7\end{array}$ & $\begin{array}{c}-138.9 \\
-138.9 \\
53.3 \\
49.2\end{array}$ & & $\begin{array}{c}0.22 \\
0 \\
1.04 \\
1.32\end{array}$ \\
\hline $\begin{array}{l}\text { Di- } \\
\text { mer } \\
\text { of } \\
\text { tetra } \\
\text { amide }\end{array}$ & $\begin{array}{l}\text { Anti_chair } \\
\text { Anti_boat } \\
\text { Anti_chair_boat } \\
\text { Syn_chair } \\
\text { Syn_boat } \\
\text { Syn_boat_chair } \\
\text { Syn_chair_boat }\end{array}$ & $\begin{array}{c}-138.2 \\
(-137.1) \\
-134.4 \\
(-133.0) \\
-131.7 \\
(-132.3) \\
-137.4 \\
(-137.2) \\
-135.2 \\
(-133.9) \\
-137.9 \\
(-134.3) \\
-138.7 \\
(-137.3)\end{array}$ & $\begin{array}{c}44.9 \\
(40.5) \\
49.4 \\
(42.2) \\
53.5 \\
(38.6) \\
\\
-138.9 \\
(-141.8) \\
-140.7 \\
(-141.4) \\
-138.5 \\
(-141.2) \\
-135.1 \\
(-142.4)\end{array}$ & $\begin{array}{c}44.6 \\
(52.6) \\
45.0 \\
(47.7) \\
39.9 \\
(47.9) \\
\\
38.2 \\
(51.3) \\
40.0 \\
(47.2) \\
39.3 \\
(47.5) \\
34.3 \\
(52.0)\end{array}$ & $\begin{array}{c}-132.0 \\
(-131.6) \\
-138.1 \\
(-137.5) \\
-138.0 \\
(-136.8) \\
\\
43.9 \\
(54.7) \\
42.0 \\
(49.0) \\
43.1 \\
(49.0) \\
43.9 \\
(54.5) \\
\end{array}$ & & $\begin{array}{c}0.81 \\
0 \\
1.45 \\
\\
7.76 \\
6.19 \\
6.59 \\
7.04\end{array}$ \\
\hline
\end{tabular}




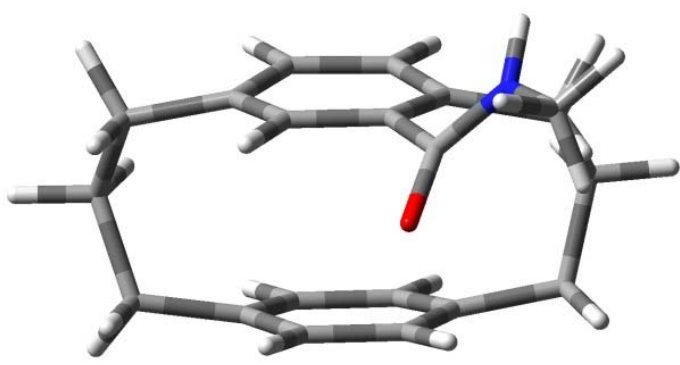

Boat_anti_1

Energy: -905.614017349 a.u.

Dihedral Angle: $45.8^{\circ}$

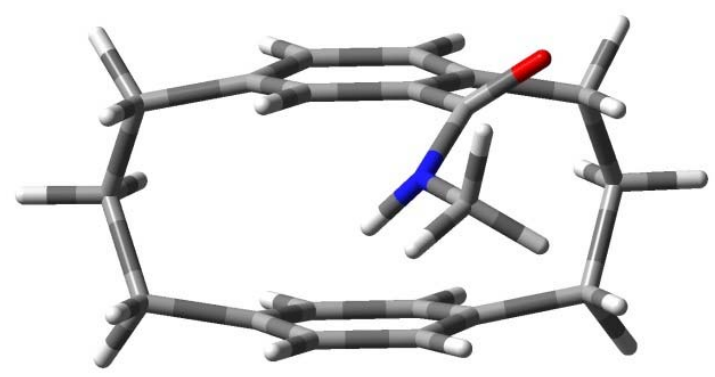

Boat_anti_3

Energy: -905.613277864 a.u.

Dihedral Angle: -140.3 ${ }^{\circ}$

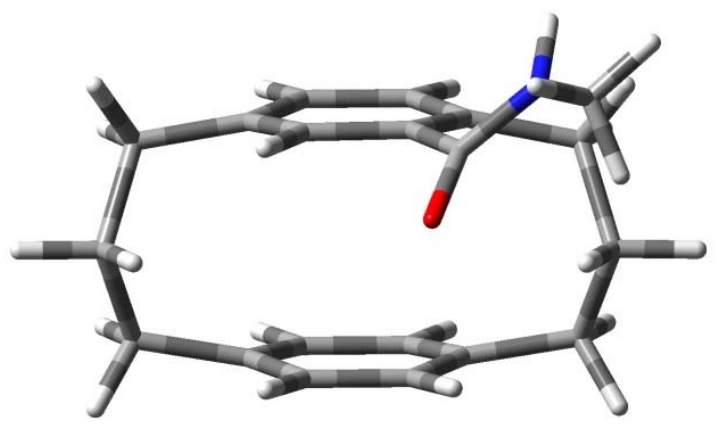

Boat_syn_1

Energy: -905.614745858 a.u.

Dihedral Angle: $44.4^{\circ}$

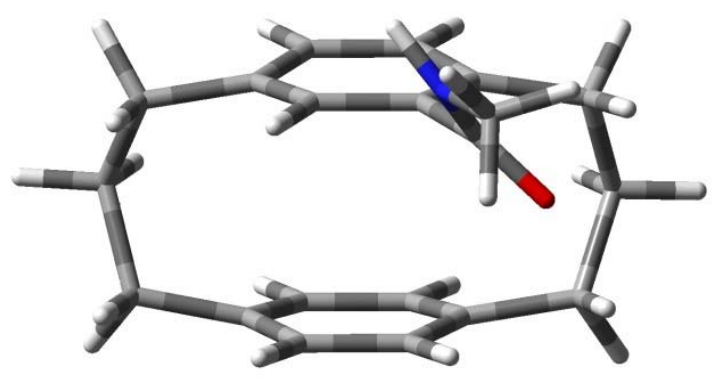

Boat_anti_2

Energy: -905.615567091 a.u.

Dihedral Angle: $129.8^{\circ}$

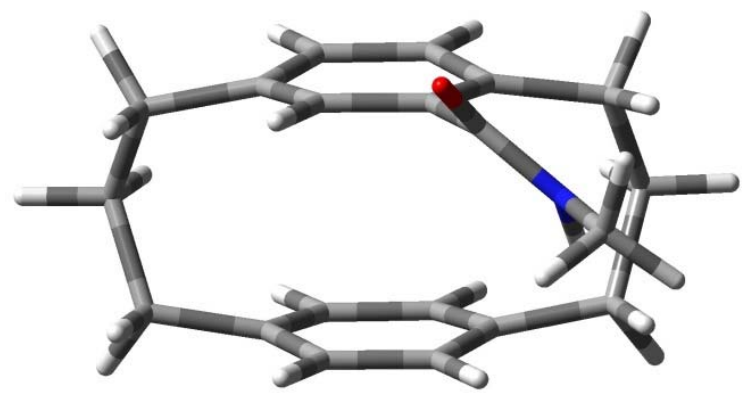

Boat_anti_4

Energy: -905.608826909 a.u.

Dihedral Angle: $-43.0^{\circ}$

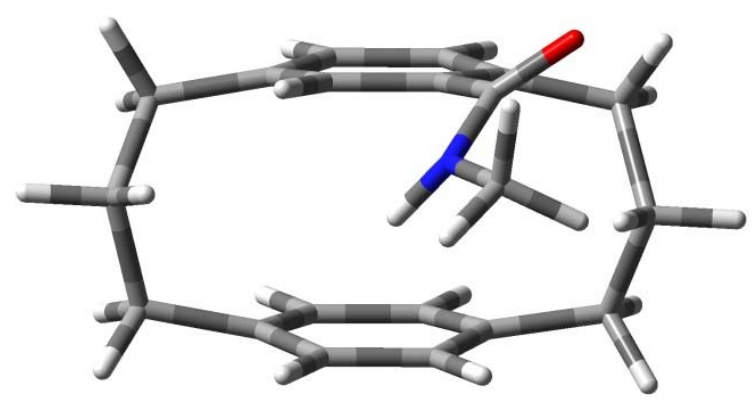

Boat_syn_2

Energy: -905.615860804 a.u.

Dihedral Angle: $-138.9^{\circ}$ 


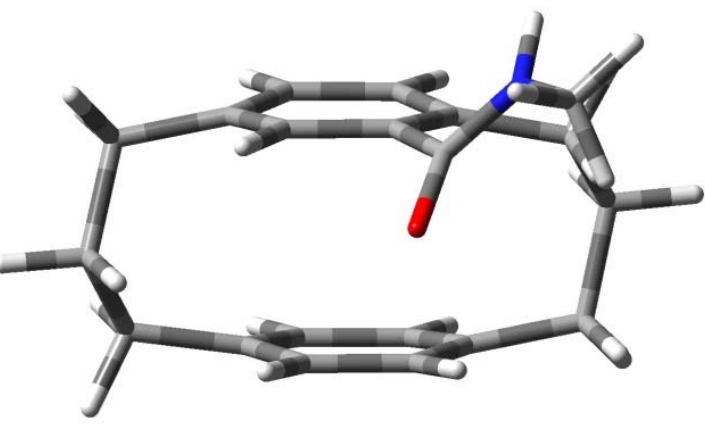

Chair anti_1

Energy: -905. $\overline{6} 136 \overline{5} 2218$ a.u.

Dihedral Angle: $48.2^{\circ}$

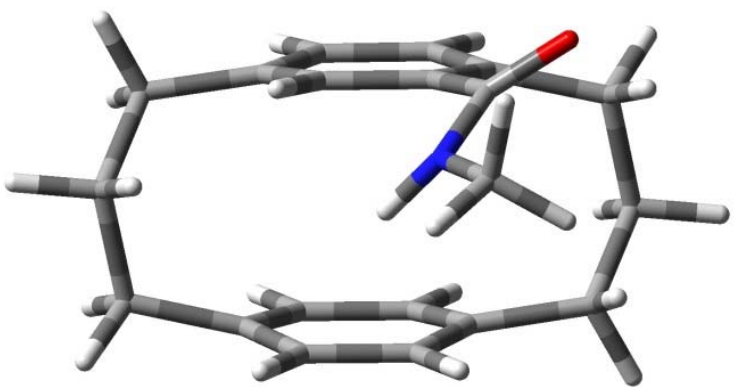

Chair anti 3

Energy: $-905 . \overline{6} 135 \overline{6} 6869$ a.u.

Dihedral Angle: $-141.0^{\circ}$

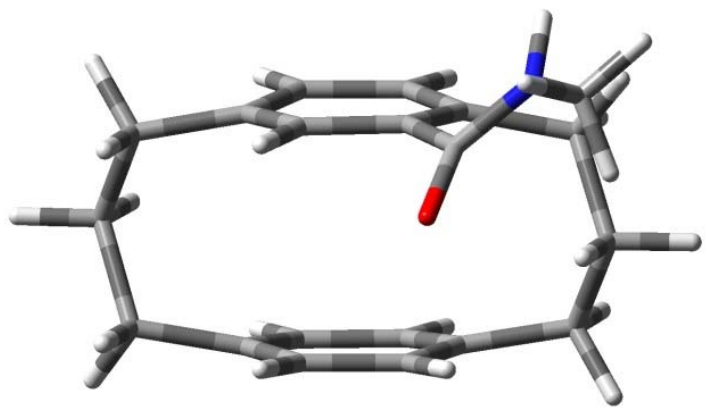

Chair_syn_1 Energy: -905. $\overline{6} 148 \overline{7} 5435$ a.u.

Dihedral Angle: $44.6^{\circ}$

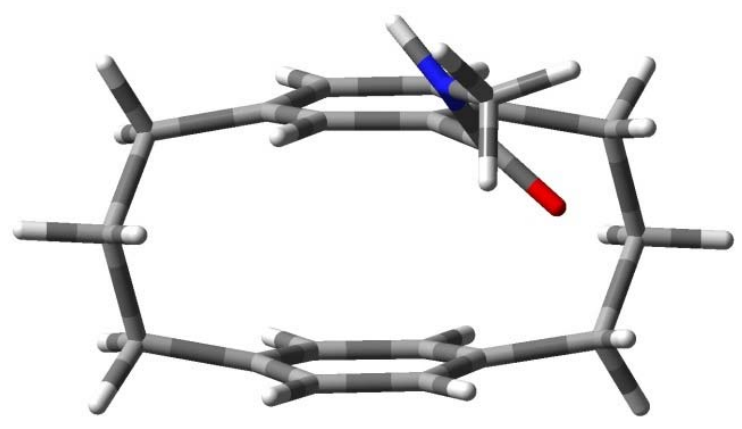

Chair anti 2 Energy: $-905 . \overline{6} 158 \overline{2} 8667$ a.u.

Dihedral Angle: $130.8^{\circ}$

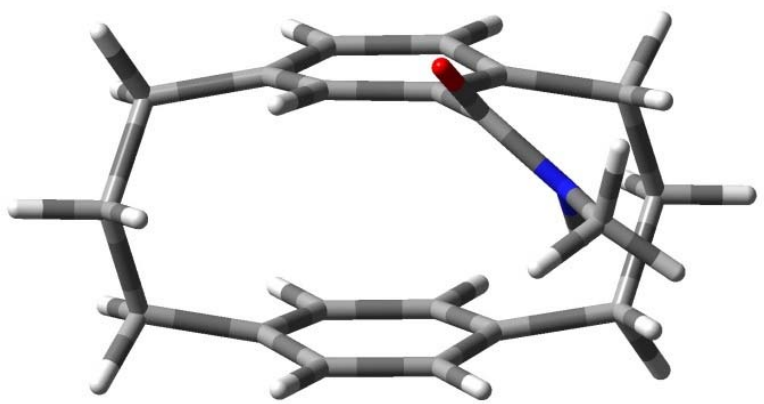

Chair anti 4 Energy: $-905 . \overline{6} 08855407$ a.u.

Dihedral Angle: $-43.5^{\circ}$

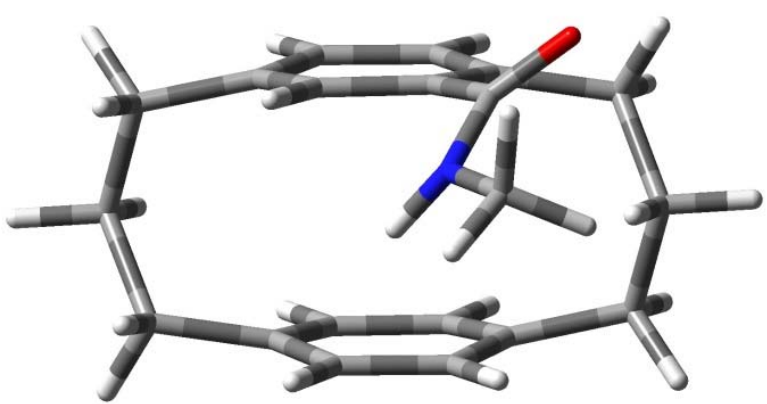

Chair_syn_2 Energy: -905.615388596 a.u.

Dihedral Angle: -137.5

Figure S5. Optimized structures of $(R \mathrm{p})$-[3.3]pCp-5-monocarboxamide. Dihedral angles and calculated energies are shown. 


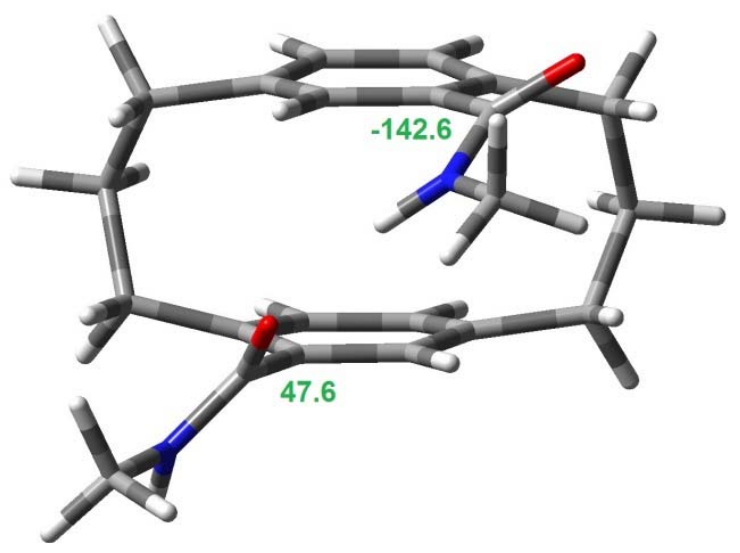

Pseudoortho_1

Energy: -1113.56152192 a.u.

Dihedral Angles: $-142.6^{\circ}, 47.6^{\circ}$

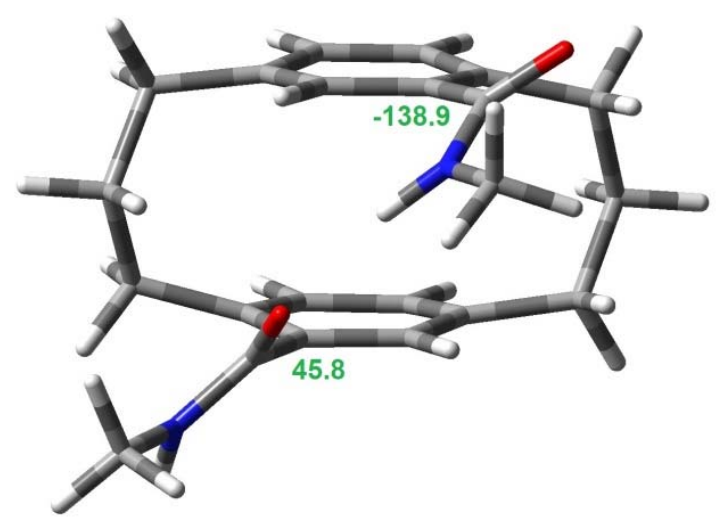

Pseudoortho_3

Energy: -1113.56232158 a.u.

Dihedral Angles: $-138.9^{\circ}, 45.8^{\circ}$

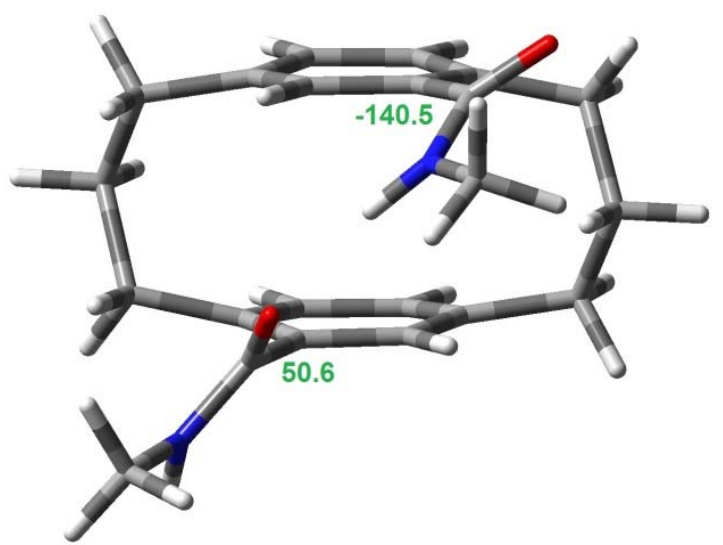

Pseudoortho_2

Energy: -1113.56340448 a.u.

Dihedral Angles: $-140.5^{\circ}, 50.6^{\circ}$

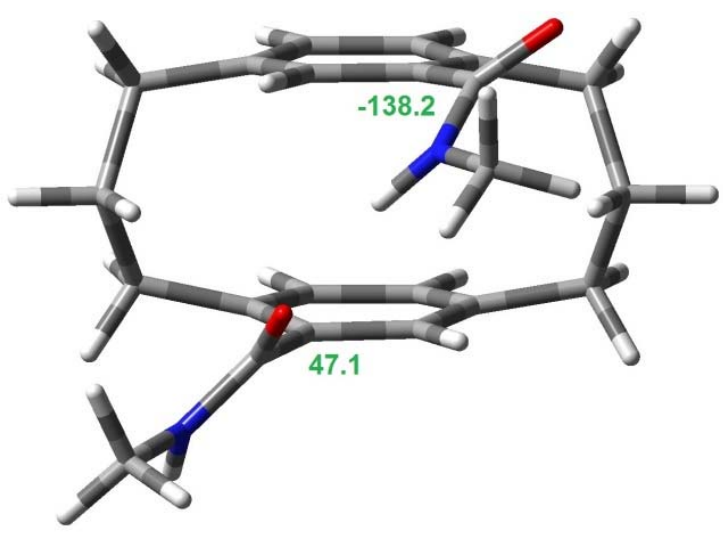

Pseudoortho_4

Energy: -1113.56481113 a.u.

Dihedral Angles: $-138.2^{\circ}, 47.1^{\circ}$

Figure S6. Optimized structures of "pseudo-ortho" [3.3]pCp-5,14-dicarboxamide. 


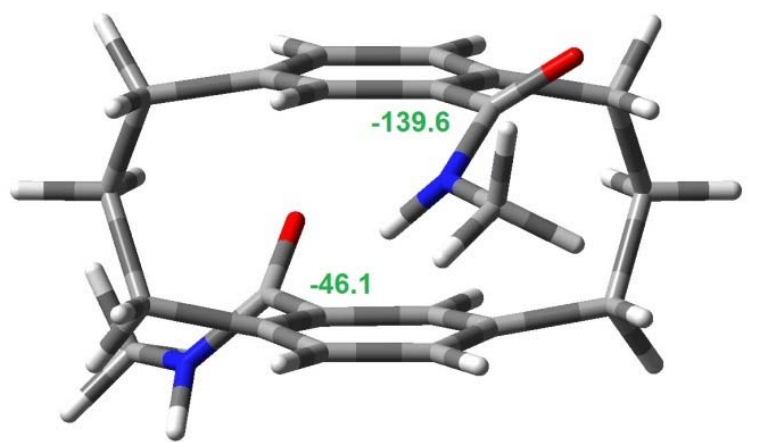

Pseudopara_1

Energy: -1113.55180581 a.u.

Dihedral Angles: $-139.6^{\circ},-46.1^{\circ}$

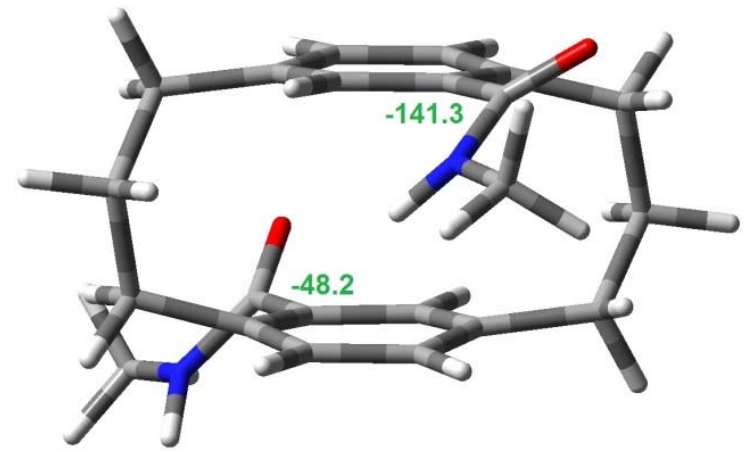

Pseudopara_3

Energy: -1113.55054520 a.u.

Dihedral Angles: $-141.3^{\circ},-48.2^{\circ}$

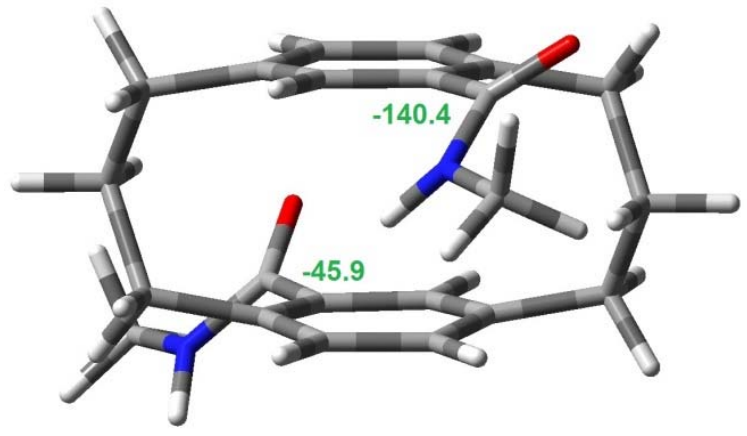

Pseudopara_2

Energy: -1113.55389537 a.u.

Dihedral Angles: $-140.4^{\circ},-45.9^{\circ}$

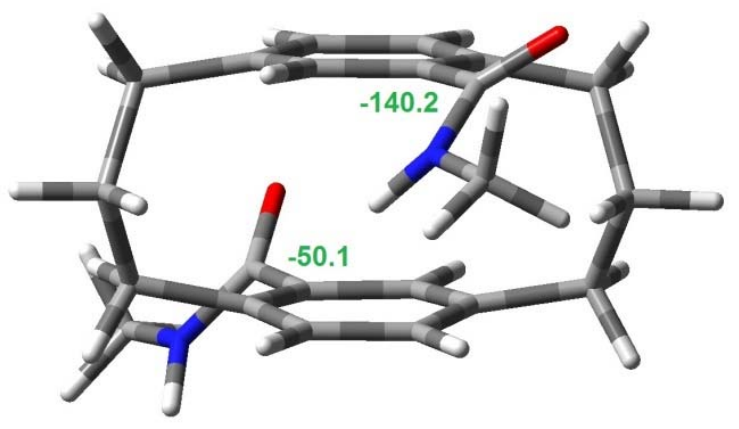

Pseudopara_4

Energy: -1113.55327708 a.u.

Dihedral Angles: $-140.2^{\circ},-50.1^{\circ}$

Figure S7. Optimized structures of "pseudo-para" [3.3]pCp-5,18-dicarboxamide. 


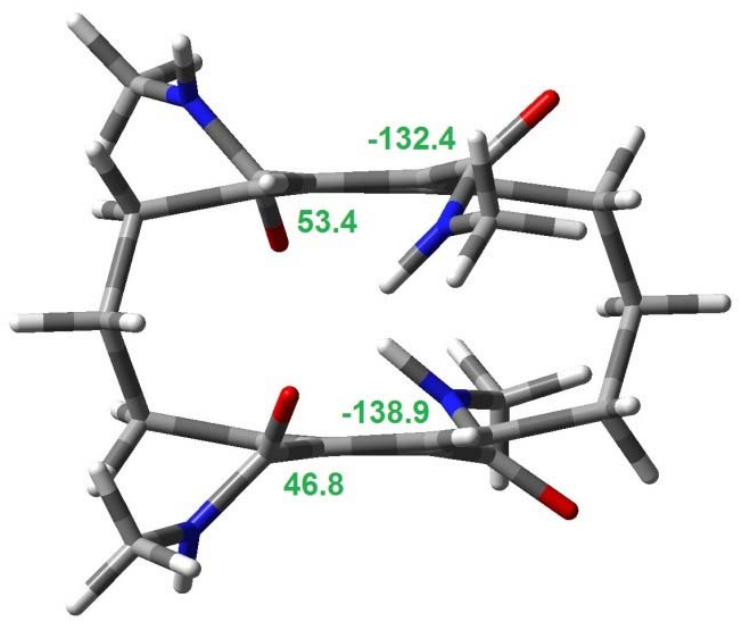

Anti chair

Energy: -1529.44961429 a.u.

Dihedral Angles: $-132.4^{\circ}, 53.4^{\circ}, 46.8^{\circ}$, $138.9^{\circ}$

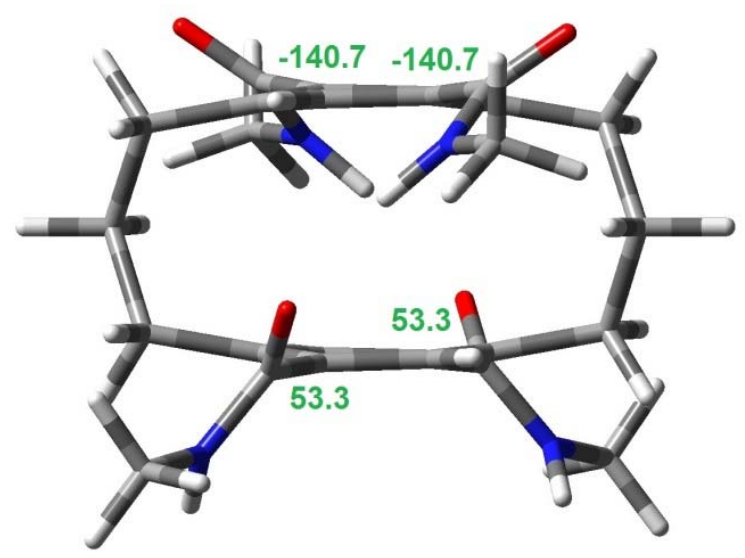

Syn_chair

Energy: -1529.44830413 a.u.

Dihedral Angles: $-140.7^{\circ},-140.7^{\circ}, 53.3^{\circ}$, $53.3^{\circ}$

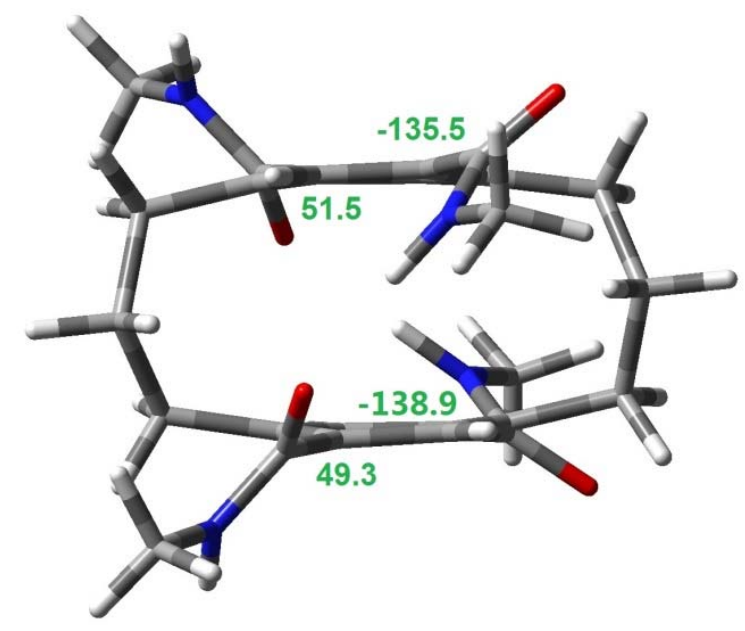

Anti boat Energy: -1529.44996641 a.u.

Dihedral Angles: $-135.5^{\circ}, 51.5^{\circ}, 49.3^{\circ},-138.9^{\circ}$

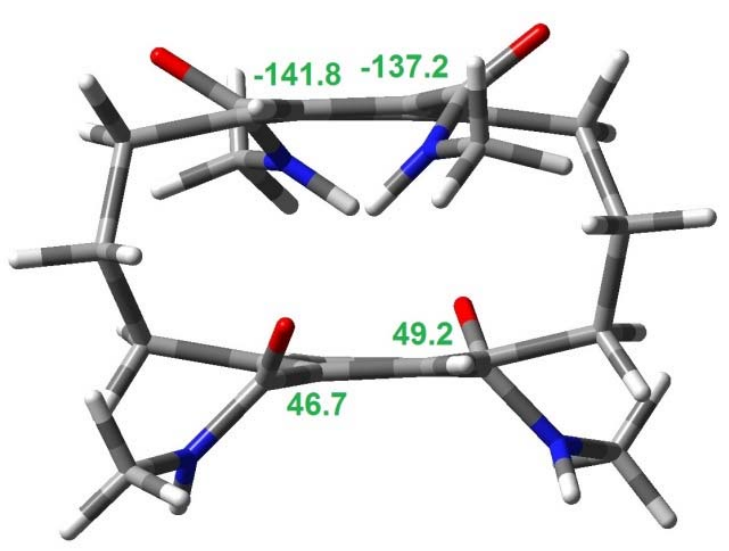

Syn boat

Energy: -1529.44785218 a.u.

Dihedral Angles: $-137.2^{\circ},-141.8^{\circ}, 46.7^{\circ}, 49.2^{\circ}$

Figure S8. Optimized structures of $(R \mathrm{p})-[3.3] \mathrm{pCp}-5,8,14,17$-tetracarboxamide monomers. 


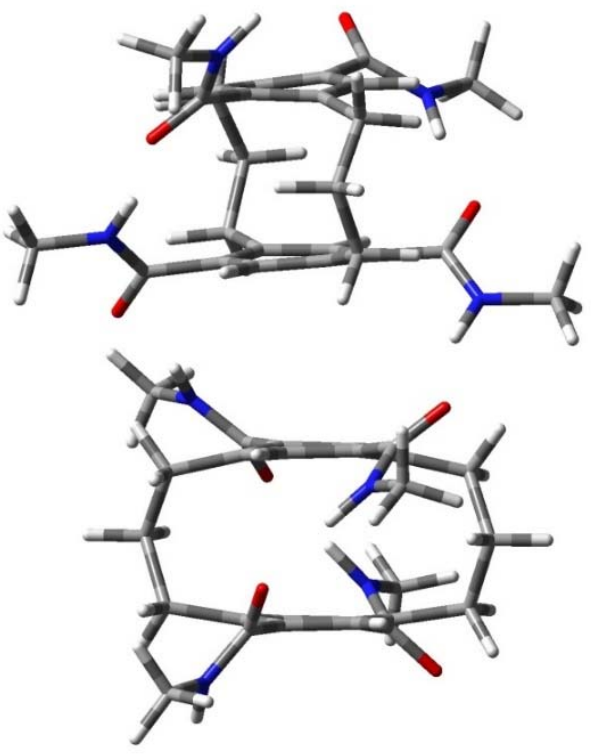

Anti_chair

Energy: -3058.94753552 a.u.

Dihedral Angles: $-138.2^{\circ}, 44.9^{\circ}, 44.6^{\circ}$, 132.0 ;

$-137.1^{\mathrm{o}}, 40.5^{\mathrm{o}}, 52.6^{\mathrm{o}},-131.6^{\circ}$

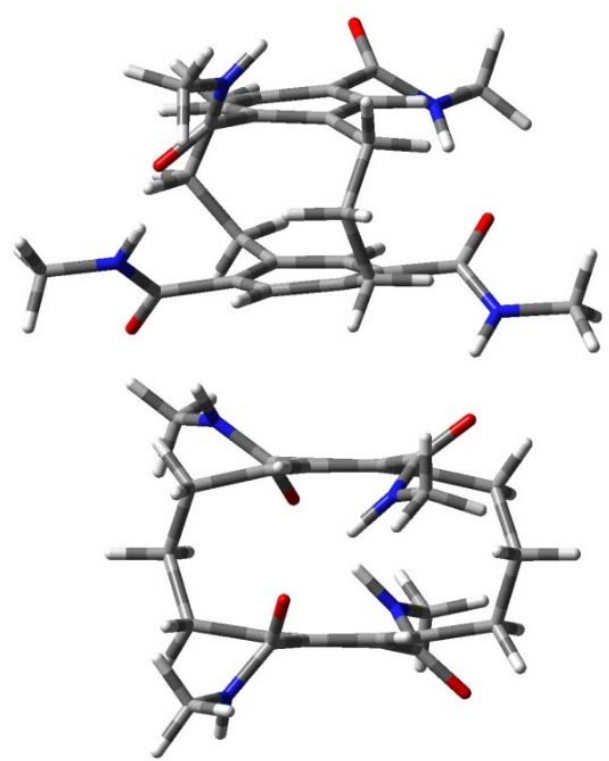

Anti_chair_boat

Energy: -3058.94652133 a.u.

Dihedral Angles: $-131.7^{\circ}, 53.5^{\circ}, 39.9^{\circ}$, 138.0

$-132.3^{\circ}, 38.6^{\circ}, 47.9^{\circ},-136.8^{\circ}$

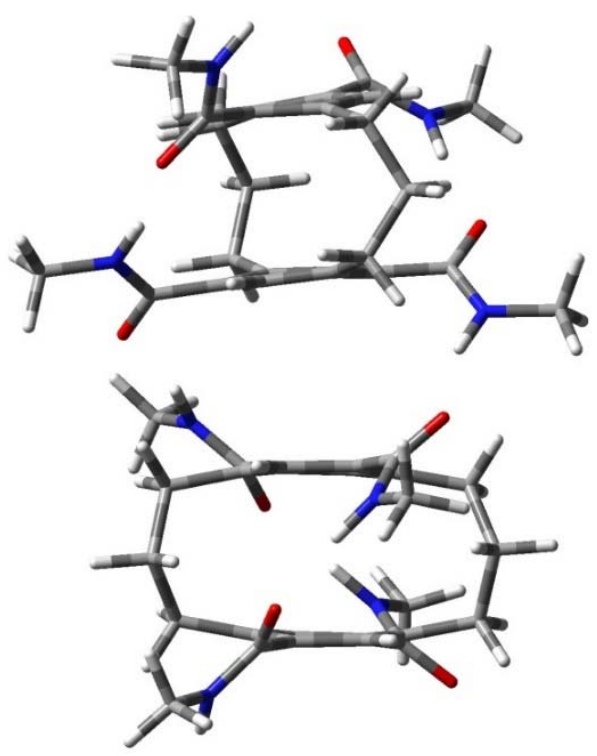

Anti_boat Energy: -3058.94883700 a.u. Dihedral Angles: $-134.4^{\circ}, 49.4^{\circ}, 45.0^{\circ}$, 138. $1^{\circ}$;

$-133.0^{\circ}, 42.2^{\circ}, 47.7^{\circ},-137.5^{\circ}$ 

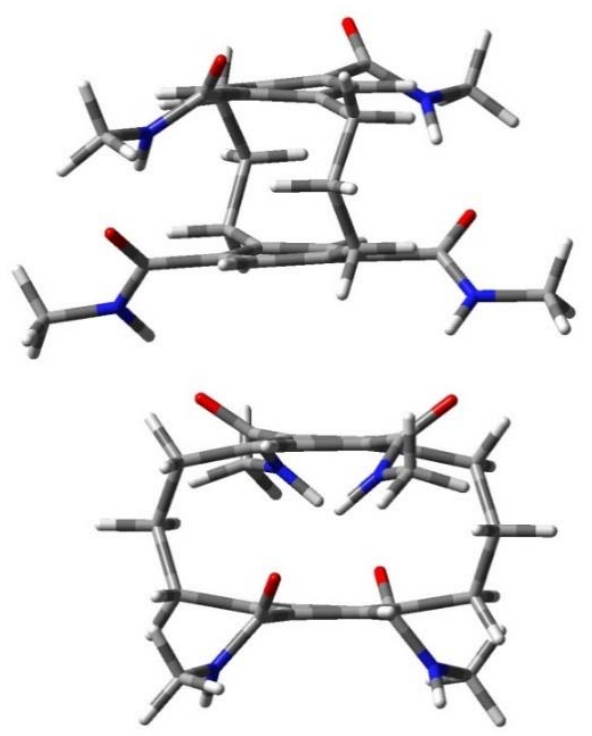

Syn_chair

Energy: -3058.93646342 a.u.

Dihedral Angles: $-137.4^{\circ},-138.9^{\circ}, 38.2^{\circ}$, $43.9^{\circ}$;
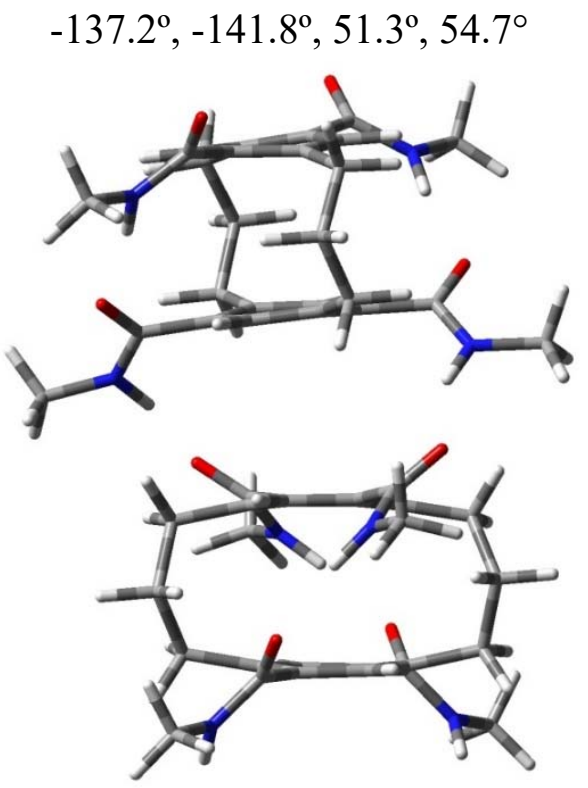

Syn_boat_chair

Energy: -3058.93833198 a.u.

Dihedral Angles: $-137.9^{\circ},-138.5^{\circ}, 39.3^{\circ}$, 43.1 ${ }^{\circ}$;

$$
-134.3^{\circ},-141.2^{\circ}, 47.5^{\circ}, 49.0^{\circ}
$$
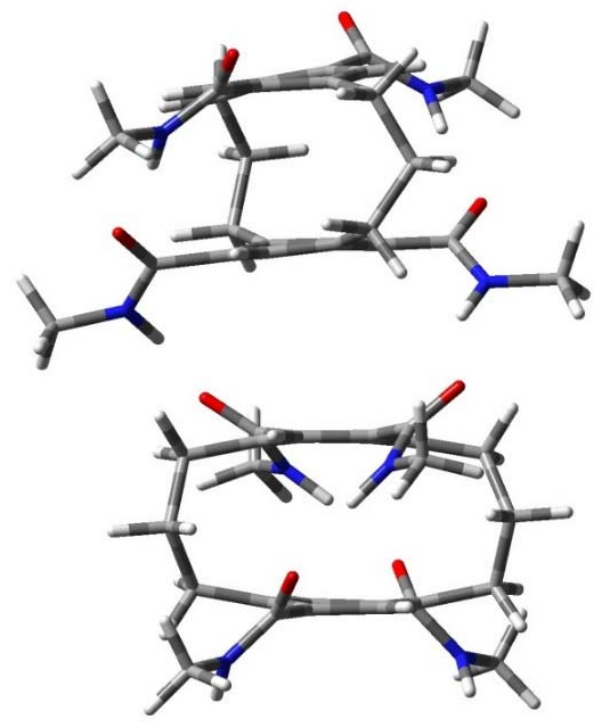

Syn boat

Energy: -3058.93897400 a.u.

Dihedral Angles: $-135.2^{\circ},-140.7^{\circ}, 40.0^{\circ}$, $42.0^{\circ}$;

$$
-133.9^{\circ},-141.4^{\circ}, 47.2^{\circ}, 49.0^{\circ}
$$
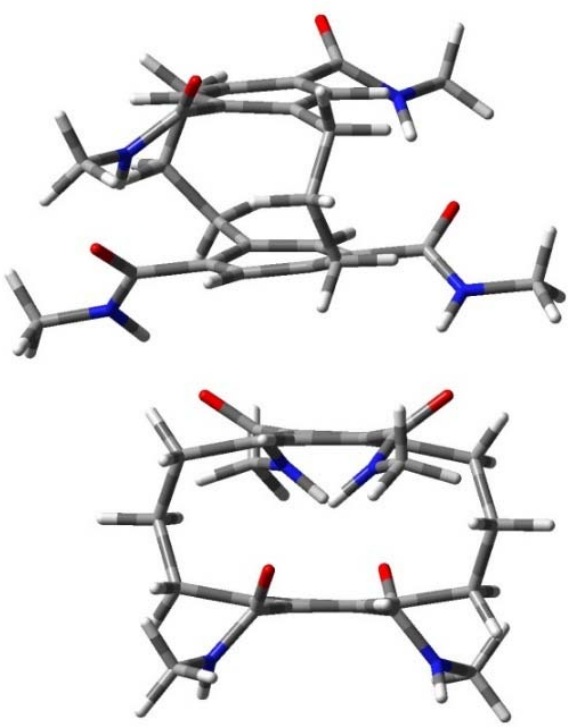

Syn_chair_boat

Energy: -3058.93761031 a.u.

Dihedral Angles: $-138.7^{\circ},-135.1^{\circ}, 34.3^{\circ}$, 43.9;

$-137.3^{\circ},-142.4^{\circ}, 52.0^{\circ}, 54.5^{\circ}$

Figure S9. Optimized structures of $(R \mathrm{p})$-[3.3]pCp-5, 8, 14, 17-tetracarboxamide dimers. 


\section{Cartesian Coordinates of Optimized pCp Structures}

$\left(\boldsymbol{R}_{\mathrm{p}}\right)$-[3.3]pCp-5-monocarboxamide (Boat_anti_1), dihedral $=45.8^{\circ}$

Total Energy: -905.614017349 a.u.

$\begin{array}{rrrr}\mathrm{C} & 0.000000 & 0.000000 & 0.000000 \\ \mathrm{C} & 0.000000 & 0.000000 & 1.400554 \\ \mathrm{C} & 1.232374 & 0.000000 & 2.051548 \\ \mathrm{C} & 2.422627 & 0.115825 & 1.336367 \\ \mathrm{C} & 2.414926 & 0.240756 & -0.051154 \\ \mathrm{C} & 1.187821 & 0.120045 & -0.715215 \\ \mathrm{C} & -1.293994 & 0.095091 & 2.179717 \\ \mathrm{C} & -2.022342 & 1.447516 & 2.036375 \\ \mathrm{C} & -1.351403 & 2.665921 & 2.703900 \\ \mathrm{C} & -0.028395 & 3.055191 & 2.082302 \\ \mathrm{C} & 1.155421 & 3.043700 & 2.809449 \\ \mathrm{C} & 2.401217 & 3.178168 & 2.185518 \\ \mathrm{C} & 2.492382 & 3.288097 & 0.790697 \\ \mathrm{C} & 1.290976 & 3.443993 & 0.087186 \\ \mathrm{C} & 0.059079 & 3.339342 & 0.715001 \\ \mathrm{C} & 3.793037 & 3.123221 & 0.015805 \\ \mathrm{C} & 3.734868 & 2.126036 & -1.162191 \\ \mathrm{C} & 3.673238 & 0.629457 & -0.791141 \\ \mathrm{C} & 3.597520 & 3.047240 & 3.084397 \\ \mathrm{~N} & 4.557174 & 4.000051 & 2.943098 \\ \mathrm{C} & 5.726584 & 4.004144 & 3.800886 \\ \mathrm{O} & 3.697849 & 2.150925 & 3.914482 \\ \mathrm{H} & 1.318490 & 3.576503 & -0.991765 \\ \mathrm{H} & -0.847985 & 3.400855 & 0.116424 \\ \mathrm{H} & 1.139826 & 2.840097 & 3.878356 \\ \mathrm{H} & 3.360987 & 0.191403 & 1.883134 \\ \mathrm{H} & 1.271545 & -0.011147 & 3.139125 \\ \mathrm{H} & -0.947157 & -0.045778 & -0.536217 \\ \mathrm{H} & 1.158092 & 0.171470 & -1.803003 \\ \mathrm{H} & -1.093777 & -0.094662 & 3.242269 \\ \mathrm{H} & -1.983298 & -0.691214 & 1.845358 \\ \mathrm{H} & -2.051904 & 3.509800 & 2.645688 \\ \mathrm{H} & -1.202402 & 2.456579 & 3.770833 \\ \mathrm{H} & 4.592922 & 2.807908 & 0.694819 \\ \mathrm{H} & 4.102694 & 4.097831 & -0.388495 \\ \mathrm{H} & 3.758105 & 0.051330 & -1.720193 \\ \mathrm{H} & 4.549259 & 0.377679 & -0.179679 \\ \mathrm{H} & 4.638848 & 2.280737 & -1.764449 \\ \mathrm{H} & 2.888737 & 2.364597 & -1.817688 \\ \mathrm{H} & -3.022957 & 1.340080 & 2.473330 \\ \mathrm{H} & -2.175222 & 1.656832 & 0.969176 \\ \mathrm{H} & 4.370137 & 4.789592 & 2.341748 \\ \mathrm{H} & 6.417514 & 4.773404 & 3.452862 \\ \mathrm{H} & 5.453789 & 4.201477 & 4.842912 \\ \mathrm{H} & 6.218155 & 3.029177 & 3.758344 \\ & & & \end{array}$

$\left(\boldsymbol{R}_{\mathrm{p}}\right)$-[3.3]pCp-5-monocarboxamide (Boat_anti_2), dihedral $=129.8^{\circ}$

Total Energy: -905.615567091 a.u.

$\begin{array}{lrrr}\mathrm{C} & 0.000000 & 0.000000 & 0.000000 \\ \mathrm{C} & 0.000000 & 0.000000 & 1.399502 \\ \mathrm{C} & 1.234090 & 0.000000 & 2.047194 \\ \mathrm{C} & 2.422238 & 0.156147 & 1.332017 \\ \mathrm{C} & 2.407822 & 0.313682 & -0.054040 \\ \mathrm{C} & 1.182796 & 0.153000 & -0.713210 \\ \mathrm{C} & -1.297560 & 0.122084 & 2.166555\end{array}$




$\begin{array}{rrrr}\mathrm{C} & -2.055396 & 1.437708 & 1.894333 \\ \mathrm{C} & -1.377978 & 2.741423 & 2.367086 \\ \mathrm{C} & -0.145812 & 3.142070 & 1.582955 \\ \mathrm{C} & 1.103356 & 3.224951 & 2.189109 \\ \mathrm{C} & 2.280299 & 3.367181 & 1.441090 \\ \mathrm{C} & 2.224584 & 3.438348 & 0.043287 \\ \mathrm{C} & 0.954319 & 3.525735 & -0.540809 \\ \mathrm{C} & -0.206969 & 3.374836 & 0.204953 \\ \mathrm{C} & 3.434991 & 3.343579 & -0.860140 \\ \mathrm{C} & 3.501061 & 2.013613 & -1.642897 \\ \mathrm{C} & 3.654639 & 0.721199 & -0.813821 \\ \mathrm{C} & 3.587923 & 3.309308 & 2.176906 \\ \mathrm{~N} & 3.655187 & 4.090132 & 3.289557 \\ \mathrm{C} & 4.866056 & 4.131441 & 4.086779 \\ \mathrm{O} & 4.527770 & 2.595634 & 1.841647 \\ \mathrm{H} & 0.883006 & 3.645928 & -1.620700 \\ \mathrm{H} & -1.171249 & 3.373395 & -0.300735 \\ \mathrm{H} & 1.182257 & 3.063676 & 3.263362 \\ \mathrm{H} & 3.365748 & 0.248889 & 1.864653 \\ \mathrm{H} & 1.265978 & -0.036012 & 3.135289 \\ \mathrm{H} & -0.945833 & -0.054371 & -0.537692 \\ \mathrm{H} & 1.144197 & 0.211644 & -1.799857 \\ \mathrm{H} & -1.096159 & 0.037434 & 3.242551 \\ \mathrm{H} & -1.968176 & -0.707981 & 1.907431 \\ \mathrm{H} & -2.124094 & 3.544900 & 2.305564 \\ \mathrm{H} & -1.112581 & 2.643816 & 3.428011 \\ \mathrm{H} & 4.356858 & 3.468914 & -0.289627 \\ \mathrm{H} & 3.387075 & 4.156229 & -1.596979 \\ \mathrm{H} & 3.937622 & -0.083639 & -1.505963 \\ \mathrm{H} & 4.480850 & 0.846206 & -0.105258 \\ \mathrm{H} & 4.358645 & 2.081961 & -2.323566 \\ \mathrm{H} & 2.610272 & 1.933359 & -2.280536 \\ \mathrm{H} & -3.030785 & 1.372208 & 2.392503 \\ \mathrm{H} & -2.266751 & 1.511659 & 0.819343 \\ \mathrm{H} & 2.928310 & 4.773395 & 3.445703 \\ \mathrm{H} & 5.702562 & 4.551676 & 3.518397 \\ \mathrm{H} & 4.684151 & 4.741868 & 4.972543 \\ \mathrm{H} & 5.139752 & 3.119282 & 4.394257 \\ & & & \end{array}$

$\left(\boldsymbol{R}_{\mathbf{p}}\right)$-[3.3]pCp-5-monocarboxamide (Boat_anti_3), dihedral $=-140.3^{\circ}$ Total Energy: -905.613277864 a.u.

$\begin{array}{rrrr}\mathrm{C} & 0.000193 & -0.000232 & 0.000254 \\ \mathrm{C} & 0.000105 & 0.000076 & 1.400266 \\ \mathrm{C} & 1.236300 & 0.000432 & 2.044571 \\ \mathrm{C} & 2.422501 & 0.157938 & 1.325868 \\ \mathrm{C} & 2.404996 & 0.326772 & -0.057691 \\ \mathrm{C} & 1.180058 & 0.161501 & -0.715031 \\ \mathrm{C} & -1.298866 & 0.092329 & 2.171999 \\ \mathrm{C} & -2.131660 & 1.356660 & 1.869739 \\ \mathrm{C} & -1.558666 & 2.705377 & 2.352328 \\ \mathrm{C} & -0.292872 & 3.130585 & 1.641568 \\ \mathrm{C} & 0.913571 & 3.254756 & 2.321473 \\ \mathrm{C} & 2.131170 & 3.426343 & 1.649540 \\ \mathrm{C} & 2.159783 & 3.470690 & 0.248046 \\ \mathrm{C} & 0.927515 & 3.498078 & -0.416314 \\ \mathrm{C} & -0.274400 & 3.333090 & 0.258321 \\ \mathrm{C} & 3.431735 & 3.378081 & -0.573433 \\ \mathrm{C} & 3.495340 & 2.145882 & -1.500000 \\ \mathrm{C} & 3.637071 & 0.768264 & -0.817274 \\ \mathrm{C} & 3.397370 & 3.491366 & 2.456734 \\ \mathrm{~N} & 3.446992 & 2.646543 & 3.527417 \\ \mathrm{C} & 4.633249 & 2.587901 & 4.358758\end{array}$




$\begin{array}{rrrr}\mathrm{O} & 4.317704 & 4.259363 & 2.206932 \\ \mathrm{H} & 0.916804 & 3.584041 & -1.501497 \\ \mathrm{H} & -1.204724 & 3.297823 & -0.306372 \\ \mathrm{H} & 0.915838 & 3.177226 & 3.407583 \\ \mathrm{H} & 3.371911 & 0.224862 & 1.855224 \\ \mathrm{H} & 1.267059 & -0.058741 & 3.132754 \\ \mathrm{H} & -0.945374 & -0.059811 & -0.536520 \\ \mathrm{H} & 1.140875 & 0.228720 & -1.801197 \\ \mathrm{H} & -1.086729 & 0.048948 & 3.248519 \\ \mathrm{H} & -1.926419 & -0.779447 & 1.944071 \\ \mathrm{H} & -2.337163 & 3.466687 & 2.211238 \\ \mathrm{H} & -1.367909 & 2.650981 & 3.431995 \\ \mathrm{H} & 4.307266 & 3.391271 & 0.079058 \\ \mathrm{H} & 3.510833 & 4.274374 & -1.202385 \\ \mathrm{H} & 3.873886 & 0.031457 & -1.596140 \\ \mathrm{H} & 4.496426 & 0.791197 & -0.134729 \\ \mathrm{H} & 4.363552 & 2.278663 & -2.157202 \\ \mathrm{H} & 2.616938 & 2.128509 & -2.158524 \\ \mathrm{H} & -3.114092 & 1.227282 & 2.340442 \\ \mathrm{H} & -2.318066 & 1.414324 & 0.789195 \\ \mathrm{H} & 2.769289 & 1.898865 & 3.576576 \\ \mathrm{H} & 4.926775 & 3.602300 & 4.636007 \\ \mathrm{H} & 4.406930 & 2.016752 & 5.260802 \\ \mathrm{H} & 5.475050 & 2.122553 & 3.832690\end{array}$

$\left(\boldsymbol{R}_{\mathbf{p}}\right)$-[3.3]pCp-5-monocarboxamide (Boat_anti_4), dihedral $=-43.0^{\circ}$ Total Energy: -905.608826909 a.u.

$\begin{array}{rrrr}\mathrm{C} & 0.000000 & 0.000000 & 0.000000 \\ \mathrm{C} & 0.000000 & 0.000000 & 1.400532 \\ \mathrm{C} & 1.234946 & 0.000000 & 2.045159 \\ \mathrm{C} & 2.421611 & 0.146160 & 1.325459 \\ \mathrm{C} & 2.405637 & 0.322808 & -0.057075 \\ \mathrm{C} & 1.179455 & 0.163550 & -0.714535 \\ \mathrm{C} & -1.297694 & 0.083262 & 2.174776 \\ \mathrm{C} & -2.135646 & 1.345105 & 1.881377 \\ \mathrm{C} & -1.558324 & 2.693874 & 2.359850 \\ \mathrm{C} & -0.309474 & 3.128602 & 1.625181 \\ \mathrm{C} & 0.904204 & 3.287885 & 2.280928 \\ \mathrm{C} & 2.111368 & 3.440827 & 1.584408 \\ \mathrm{C} & 2.114533 & 3.458127 & 0.181453 \\ \mathrm{C} & 0.866552 & 3.483761 & -0.458238 \\ \mathrm{C} & -0.319655 & 3.316412 & 0.238169 \\ \mathrm{C} & 3.344680 & 3.415786 & -0.707446 \\ \mathrm{C} & 3.468844 & 2.125665 & -1.552681 \\ \mathrm{C} & 3.631056 & 0.768641 & -0.829459 \\ \mathrm{C} & 3.322787 & 3.546224 & 2.474278 \\ \mathrm{~N} & 4.425132 & 2.823222 & 2.129274 \\ \mathrm{C} & 5.570128 & 2.777014 & 3.019116 \\ \mathrm{O} & 3.306520 & 4.232477 & 3.487413 \\ \mathrm{H} & 0.837072 & 3.579252 & -1.542735 \\ \mathrm{H} & -1.260387 & 3.274946 & -0.308488 \\ \mathrm{H} & 0.948396 & 3.233317 & 3.366438 \\ \mathrm{H} & 3.363726 & 0.187932 & 1.869573 \\ \mathrm{H} & 1.272727 & -0.041830 & 3.131937 \\ \mathrm{H} & -0.944888 & -0.059329 & -0.537915 \\ \mathrm{H} & 1.139337 & 0.232740 & -1.800467 \\ \mathrm{H} & -1.081965 & 0.034769 & 3.249978 \\ \mathrm{H} & -1.921228 & -0.790407 & 1.942521 \\ \mathrm{H} & -2.341927 & 3.453406 & 2.237710 \\ \mathrm{H} & -1.344824 & 2.635980 & 3.434608 \\ \mathrm{H} & 4.259326 & 3.584737 & -0.134740 \\ \mathrm{H} & 3.278870 & 4.247706 & -1.420738 \\ & & & \end{array}$




$\begin{array}{rrrr}\mathrm{H} & 3.873264 & 0.021952 & -1.597325 \\ \mathrm{H} & 4.505845 & 0.795381 & -0.163673 \\ \mathrm{H} & 4.333722 & 2.254443 & -2.215161 \\ \mathrm{H} & 2.592429 & 2.057063 & -2.209932 \\ \mathrm{H} & -3.113285 & 1.212953 & 2.360998 \\ \mathrm{H} & -2.331679 & 1.401216 & 0.802429 \\ \mathrm{H} & 4.317967 & 2.104224 & 1.429404 \\ \mathrm{H} & 5.893392 & 3.795148 & 3.245263 \\ \mathrm{H} & 5.326614 & 2.280087 & 3.965088 \\ \mathrm{H} & 6.381407 & 2.242437 & 2.521725\end{array}$

$\left(\boldsymbol{R}_{\mathrm{p}}\right)$-[3.3]pCp-5-monocarboxamide (Boat_syn_1), dihedral $=44.4^{\mathrm{o}}$

Total Energy: -905.614745858 a.u.

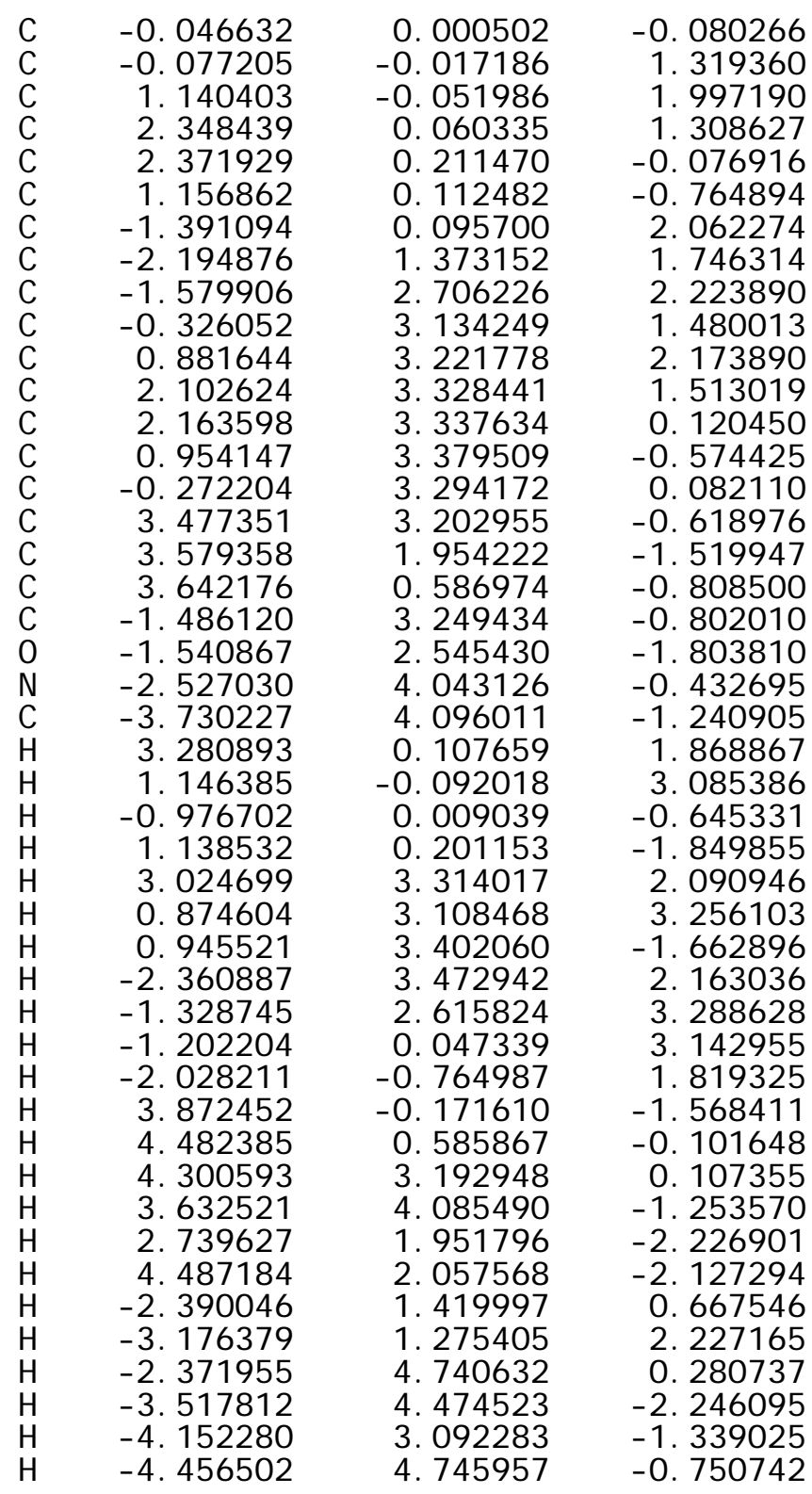

$\left(\boldsymbol{R}_{\mathbf{p}}\right)$-[3.3]pCp-5-monocarboxamide (Boat_syn_2), dihedral $=-138.9^{\circ}$

Total Energy: -905.615860804 a.u. 


$\begin{array}{rrrr}\mathrm{C} & -0.041008 & -0.157226 & -0.107569 \\ \mathrm{C} & -0.039846 & -0.017263 & 1.286647 \\ \mathrm{C} & 1.195050 & 0.098875 & 1.921312 \\ \mathrm{C} & 2.374969 & 0.210367 & 1.189645 \\ \mathrm{C} & 2.359763 & 0.215201 & -0.202870 \\ \mathrm{C} & 1.138155 & -0.039171 & -0.840669 \\ \mathrm{C} & -1.330069 & 0.074766 & 2.072967 \\ \mathrm{C} & -2.167855 & 1.341314 & 1.797258 \\ \mathrm{C} & -1.596516 & 2.688525 & 2.291882 \\ \mathrm{C} & -0.312613 & 3.111406 & 1.608745 \\ \mathrm{C} & 0.868857 & 3.251447 & 2.335032 \\ \mathrm{C} & 2.109186 & 3.354355 & 1.704766 \\ \mathrm{C} & 2.208738 & 3.318464 & 0.315418 \\ \mathrm{C} & 1.016330 & 3.348840 & -0.410161 \\ \mathrm{C} & -0.225998 & 3.250821 & 0.211767 \\ \mathrm{C} & 3.538449 & 3.177040 & -0.399930 \\ \mathrm{C} & 3.583929 & 2.070189 & -1.475093 \\ \mathrm{C} & 3.594859 & 0.607930 & -0.979064 \\ \mathrm{C} & -1.466460 & 3.249347 & -0.634541 \\ \mathrm{O} & -2.484031 & 3.858463 & -0.331523 \\ \mathrm{~N} & -1.381331 & 2.526116 & -1.787767 \\ \mathrm{C} & -2.549073 & 2.37735 & -2.634381 \\ \mathrm{H} & 3.315870 & 0.371982 & 1.713095 \\ \mathrm{H} & 1.230527 & 0.175381 & 3.006346 \\ \mathrm{H} & -0.986420 & -0.320982 & -0.624905 \\ \mathrm{H} & 1.109314 & -0.110311 & -1.928573 \\ \mathrm{H} & 3.015215 & 3.375268 & 2.307890 \\ \mathrm{H} & 0.829167 & 3.185098 & 3.420573 \\ \mathrm{H} & 1.051973 & 3.417246 & -1.495645 \\ \mathrm{H} & -2.367149 & 3.450997 & 2.142422 \\ \mathrm{H} & -1.408596 & 2.617716 & 3.371015 \\ \mathrm{H} & -1.102166 & 0.017931 & 3.145219 \\ \mathrm{H} & -1.959955 & -0.794414 & 1.841929 \\ \mathrm{H} & 3.711047 & -0.041316 & -1.856337 \\ \mathrm{H} & 4.482079 & 0.452150 & -0.351998 \\ \mathrm{H} & 4.327707 & 2.995791 & 0.341904 \\ \mathrm{H} & 3.792308 & 4.126590 & -0.890214 \\ \mathrm{H} & 2.743647 & 2.203437 & -2.168904 \\ \mathrm{H} & 4.492538 & 2.220390 & -2.071033 \\ \mathrm{H} & -2.382132 & 1.402644 & 0.722980 \\ \mathrm{H} & -3.141361 & 1.205913 & 2.284290 \\ \mathrm{H} & -0.622047 & 1.862960 & -1.869244 \\ \mathrm{H} & -2.262717 & 1.84109 & -3.540640 \\ \mathrm{H} & -3.349776 & 1.831048 & -2.121608 \\ \mathrm{H} & -2.933156 & 3.363620 & -2.903484\end{array}$

$\left(\boldsymbol{R}_{\mathbf{p}}\right)$-[3.3]pCp-5-monocarboxamide (Chair_anti_1), dihedral $=48.2^{\circ}$ Total Energy: -905.613652218 a.u.

$\begin{array}{lrrr}\mathrm{C} & -0.052006 & -0.210652 & -0.048394 \\ \mathrm{C} & -0.051955 & -0.108072 & 1.341204 \\ \mathrm{C} & 1.180892 & 0.055099 & 1.974201 \\ \mathrm{C} & 2.342526 & 0.252994 & 1.237362 \\ \mathrm{C} & 2.327951 & 0.295435 & -0.159476 \\ \mathrm{C} & 1.114121 & -0.022875 & -0.793239 \\ \mathrm{C} & -1.347063 & -0.018852 & 2.114418 \\ \mathrm{C} & -2.122669 & 1.290097 & 1.855903 \\ \mathrm{C} & -1.459048 & 2.595642 & 2.337215 \\ \mathrm{C} & -0.204496 & 3.009085 & 1.590208 \\ \mathrm{C} & -0.157427 & 2.984616 & 0.194678 \\ \mathrm{C} & 1.038203 & 3.188946 & -0.486314\end{array}$




$\begin{array}{rrrr}\mathrm{C} & 2.225516 & 3.435631 & 0.204355 \\ \mathrm{C} & 2.154755 & 3.595275 & 1.590995 \\ \mathrm{C} & 0.958627 & 3.379323 & 2.272473 \\ \mathrm{C} & 3.554859 & 3.366733 & -0.508363 \\ \mathrm{C} & 4.272715 & 2.012710 & -0.326209 \\ \mathrm{C} & 3.558864 & 0.778433 & -0.917095 \\ \mathrm{C} & 0.953449 & -0.042291 & -2.286904 \\ \mathrm{O} & 0.083705 & 0.604072 & -2.859709 \\ \mathrm{~N} & 1.819116 & -0.836830 & -2.969876 \\ \mathrm{C} & 1.740274 & -0.949890 & -4.413477 \\ \mathrm{H} & 3.266066 & 0.463025 & 1.770575 \\ \mathrm{H} & 1.222910 & 0.113486 & 3.060161 \\ \mathrm{H} & -0.987357 & -0.352767 & -0.587674 \\ \mathrm{H} & 1.054599 & 3.071664 & -1.568798 \\ \mathrm{H} & -1.043295 & 2.723366 & -0.379923 \\ \mathrm{H} & 0.942055 & 3.443934 & 3.359251 \\ \mathrm{H} & 3.059725 & 3.824054 & 2.152773 \\ \mathrm{H} & -2.208203 & 3.395218 & 2.257695 \\ \mathrm{H} & -1.222839 & 2.504805 & 3.406055 \\ \mathrm{H} & -1.139248 & -0.109911 & 3.188491 \\ \mathrm{H} & -2.002599 & -0.857229 & 1.846380 \\ \mathrm{H} & 3.283902 & 1.006672 & -1.953092 \\ \mathrm{H} & 4.294844 & -0.036997 & -0.972366 \\ \mathrm{H} & 4.224061 & 4.154253 & -0.139123 \\ \mathrm{H} & 3.408115 & 3.549899 & -1.580547 \\ \mathrm{H} & 5.251322 & 2.087764 & -0.816574 \\ \mathrm{H} & 4.480679 & 1.859303 & 0.739520 \\ \mathrm{H} & -2.345920 & 1.364427 & 0.783900 \\ \mathrm{H} & -3.093126 & 1.205864 & 2.360592 \\ \mathrm{H} & 2.448984 & -1.430036 & -2.449276 \\ \mathrm{H} & 2.574154 & -1.558605 & -4.765920 \\ \mathrm{H} & 0.795934 & -1.409564 & -4.722160 \\ \mathrm{H} & 1.798502 & 0.042600 & -4.868012\end{array}$

$\left(\boldsymbol{R}_{\mathrm{p}}\right)$-[3.3]pCp-5-monocarboxamide (Chair_anti_2), dihedral $=130.8^{\circ}$ Total Energy: -905.615828667 a.u.

$\begin{array}{lrrr}\mathrm{C} & -0.097439 & -0.155018 & 0.154965 \\ \mathrm{C} & -0.083677 & -0.078176 & 1.551992 \\ \mathrm{C} & 1.117637 & -0.014669 & 2.254915 \\ \mathrm{C} & 2.344652 & -0.038707 & 1.584600 \\ \mathrm{C} & 2.324476 & 0.027099 & 0.191914 \\ \mathrm{C} & 1.123314 & -0.023682 & -0.510841 \\ \mathrm{C} & 3.638985 & -0.272464 & 2.328208 \\ \mathrm{C} & 3.786065 & -1.709873 & 2.869289 \\ \mathrm{C} & 3.860671 & -2.843781 & 1.825343 \\ \mathrm{C} & 2.569405 & -3.140937 & 1.085935 \\ \mathrm{C} & 2.524845 & -3.238106 & -0.306699 \\ \mathrm{C} & 1.308533 & -3.303511 & -0.978979 \\ \mathrm{C} & 0.088068 & -3.245048 & -0.298539 \\ \mathrm{C} & 0.135498 & -3.316475 & 1.101703 \\ \mathrm{C} & 1.363408 & -3.273298 & 1.770773 \\ \mathrm{C} & -1.113473 & -3.322589 & 1.935685 \\ \mathrm{~N} & -1.106808 & -4.206969 & 2.971172 \\ \mathrm{C} & -2.258875 & -4.326314 & 3.843942 \\ \mathrm{C} & -1.182401 & -3.013905 & -1.086813 \\ \mathrm{C} & -1.296679 & -1.574190 & -1.635963 \\ \mathrm{C} & -1.380607 & -0.426836 & -0.608092 \\ \mathrm{O} & -2.066730 & -2.576424 & 1.740659 \\ \mathrm{H} & 1.301619 & -3.311965 & -2.067871 \\ \mathrm{H} & 3.450758 & -3.189028 & -0.876808 \\ \mathrm{H} & 1.357166 & -3.236045 & 2.858665 \\ \mathrm{H} & -1.024136 & -0.148324 & 2.092961\end{array}$




$\begin{array}{rrrr}\mathrm{H} & 1.100366 & -0.022951 & 3.344339 \\ \mathrm{H} & 3.265810 & 0.046436 & -0.355252 \\ \mathrm{H} & 1.149938 & -0.032038 & -1.598861 \\ \mathrm{H} & 3.714967 & 0.413945 & 3.181375 \\ \mathrm{H} & 4.487169 & -0.050237 & 1.667622 \\ \mathrm{H} & 4.641673 & -2.605348 & 1.091101 \\ \mathrm{H} & 4.198201 & -3.751639 & 2.343972 \\ \mathrm{H} & -2.064077 & -3.238059 & -0.483348 \\ \mathrm{H} & -1.188989 & -3.693766 & -1.948780 \\ \mathrm{H} & -1.672619 & 0.480577 & -1.154953 \\ \mathrm{H} & -2.180483 & -0.643328 & 0.107796 \\ \mathrm{H} & -2.203063 & -1.535937 & -2.252980 \\ \mathrm{H} & -0.457223 & -1.390562 & -2.319815 \\ \mathrm{H} & 2.964068 & -1.909234 & 3.568654 \\ \mathrm{H} & 4.706600 & -1.749323 & 3.465049 \\ \mathrm{H} & -0.384503 & -4.912101 & 2.998632 \\ \mathrm{H} & -3.133222 & -4.701495 & 3.301321 \\ \mathrm{H} & -2.014364 & -5.010078 & 4.658243 \\ \mathrm{H} & -2.509921 & -3.346773 & 4.257282\end{array}$

$\left(\boldsymbol{R}_{\mathrm{p}}\right)$-[3.3]pCp-5-monocarboxamide (Chair_anti_3), dihedral $=141.0^{\circ}$ Total Energy: -905.613566869 a.u.

$\begin{array}{rrrr}\mathrm{C} & 0.014894 & 0.158740 & 0.151850 \\ \mathrm{C} & 0.015654 & -0.028953 & 1.532998 \\ \mathrm{C} & 1.255671 & -0.165954 & 2.166175 \\ \mathrm{C} & 2.441646 & 0.044963 & 1.462734 \\ \mathrm{C} & 2.428921 & 0.406615 & 0.110636 \\ \mathrm{C} & 1.198839 & 0.370408 & -0.548008 \\ \mathrm{C} & -1.270090 & 0.067400 & 2.317978 \\ \mathrm{C} & -1.477649 & 1.447241 & 2.979714 \\ \mathrm{C} & -1.626336 & 2.661495 & 2.037976 \\ \mathrm{C} & 0.844694 & 3.099402 & 1.320323 \\ \mathrm{C} & 2.056284 & 3.218752 & 2.005706 \\ \mathrm{C} & 2.082815 & 3.437349 & 1.343580 \\ \mathrm{C} & 0.853122 & 3.586922 & -0.056653 \\ \mathrm{C} & -0.347483 & 3.364689 & -0.719286 \\ \mathrm{C} & 3.357409 & 3.452843 & -0.050332 \\ \mathrm{C} & 3.548117 & 2.072769 & -1.567353 \\ \mathrm{C} & 3.695333 & 0.856341 & -0.596493 \\ \mathrm{C} & 3.318161 & 3.514592 & 2.160121 \\ \mathrm{~N} & 3.373240 & 2.661742 & 3.222748 \\ \mathrm{C} & 4.567270 & 2.602228 & 4.042661 \\ \mathrm{O} & 4.225476 & 4.303819 & 1.927892 \\ \mathrm{H} & 0.845200 & 3.712578 & -1.801112 \\ \mathrm{H} & -1.277729 & 3.329188 & -0.614731 \\ \mathrm{H} & 0.854702 & 3.112919 & 3.088361 \\ \mathrm{H} & 3.396797 & -0.018915 & 1.982941 \\ \mathrm{H} & 1.291092 & -0.386669 & 3.233440 \\ \mathrm{H} & -0.934909 & 0.222735 & -0.376535 \\ \mathrm{H} & 1.145226 & 0.589989 & -1.611326 \\ \mathrm{H} & -1.288975 & -0.690995 & 3.110951 \\ \mathrm{H} & -2.120568 & -0.143308 & 1.656899 \\ \mathrm{H} & -2.400906 & 2.442662 & 1.290703 \\ \mathrm{H} & -2.010071 & 3.498901 & 2.636424 \\ \mathrm{H} & 4.222978 & 3.683325 & -0.243734 \\ \mathrm{H} & 3.332408 & 4.206684 & -1.664283 \\ \mathrm{H} & 4.091095 & 0.021583 & -1.191310 \\ \mathrm{H} & 4.460327 & 1.080892 & 0.159696 \\ \mathrm{H} & 4.460871 & 2.131279 & -2.140555 \\ \mathrm{H} & 2.730331 & 1.894044 & -2.244552 \\ \mathrm{H} & -0.655920 & 1.626726 & 3.685044 \\ & & & \end{array}$




$\begin{array}{rrrr}\mathrm{H} & -2.388721 & 1.387961 & 3.587955 \\ \mathrm{H} & 2.724728 & 1.886366 & 3.236895 \\ \mathrm{H} & 4.818928 & 3.606012 & 4.390951 \\ \mathrm{H} & 4.374371 & 1.958745 & 4.902834 \\ \mathrm{H} & 5.424900 & 2.214676 & 3.479794\end{array}$

$\left(\boldsymbol{R}_{\mathbf{p}}\right)$-[3.3]pCp-5-monocarboxamide (Chair_anti_4), dihedral $=-43.5^{\circ}$

Total Energy: -905.608855407 a.u.

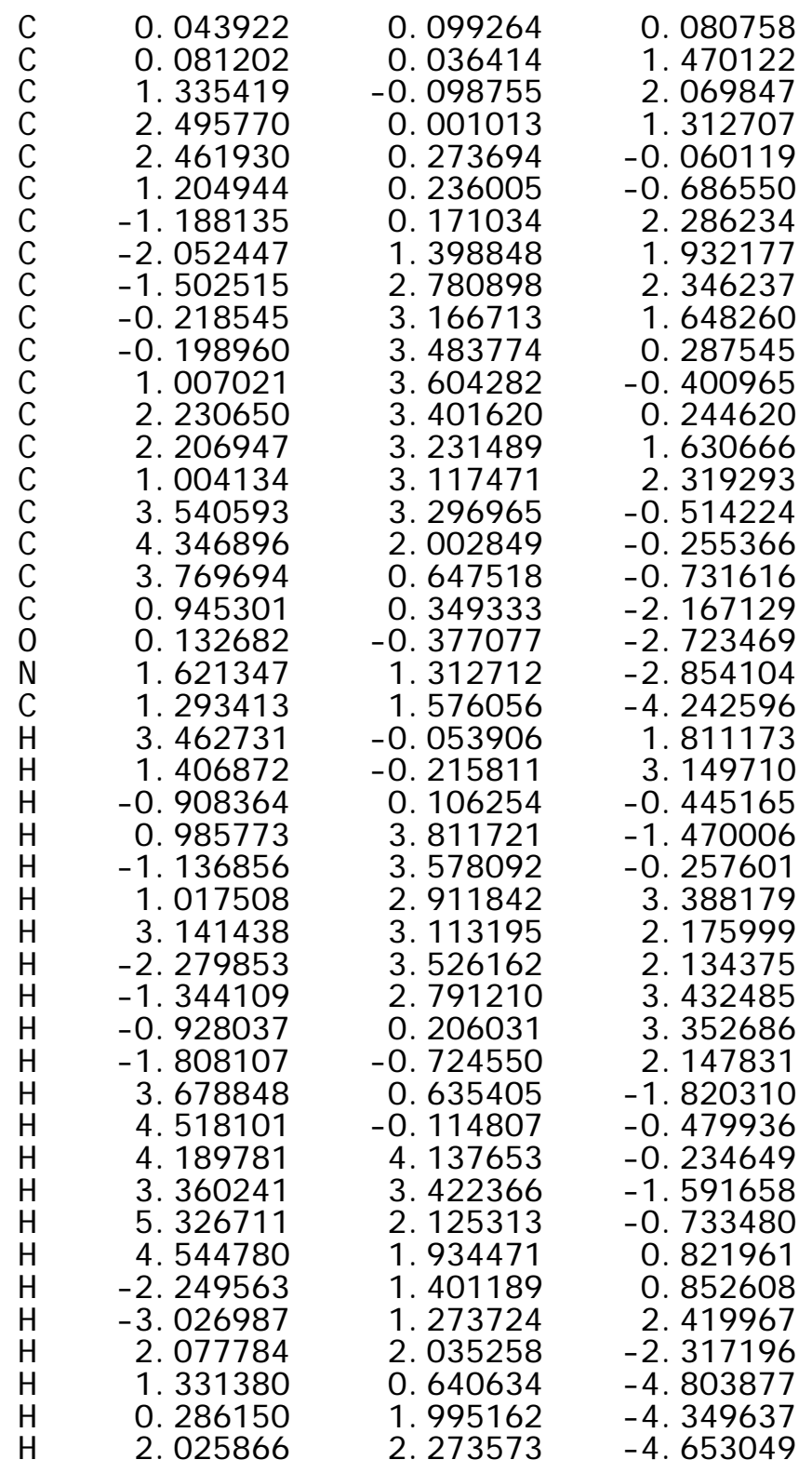

$\left(\boldsymbol{R}_{\mathrm{p}}\right)$-[3.3]pCp-5-monocarboxamide (Chair_syn_1), dihedral $=44.6^{\circ}$ Total Energy: -905.614875435 a.u.
C $\quad-0.027826$
0.013732
- 0.121078
$\begin{array}{lr}\text { C } & -0.023739 \\ \text { C } & 1.214080\end{array}$
0.000799
1. 274454
1. 923103 


$\begin{array}{rrrr}\mathrm{C} & 2.400147 & -0.086572 & 1.201003 \\ \mathrm{C} & 2.386351 & -0.201788 & -0.191810 \\ \mathrm{C} & 1.157932 & -0.077645 & -0.844595 \\ \mathrm{C} & -1.306751 & -0.153323 & 2.061114 \\ \mathrm{C} & -1.422460 & -1.496398 & 2.811567 \\ \mathrm{C} & -1.562076 & -2.765109 & 1.945112 \\ \mathrm{C} & -0.325702 & -3.120014 & 1.147615 \\ \mathrm{C} & -0.343572 & -3.154563 & -0.243735 \\ \mathrm{C} & 0.828714 & -3.282387 & -0.992738 \\ \mathrm{C} & 2.078738 & -3.340886 & -0.354585 \\ \mathrm{C} & 2.075319 & -3.425570 & 1.041515 \\ \mathrm{C} & 0.901786 & -3.332490 & 1.780129 \\ \mathrm{C} & 3.401009 & -3.150120 & -1.078042 \\ \mathrm{C} & 3.540050 & -1.811743 & -1.834391 \\ \mathrm{C} & 3.641380 & -0.538835 & -0.969653 \\ \mathrm{C} & 0.659136 & -3.206245 & -2.483462 \\ \mathrm{O} & -0.045645 & -2.358749 & -3.019820 \\ \mathrm{~N} & 1.325136 & -4.142277 & -3.211870 \\ \mathrm{C} & 1.172527 & -4.191522 & -4.653598 \\ \mathrm{H} & 3.348031 & -0.143482 & 1.733677 \\ \mathrm{H} & 1.250728 & 0.049457 & 3.011478 \\ \mathrm{H} & -0.973906 & 0.014819 & -0.659093 \\ \mathrm{H} & 1.103629 & -0.145315 & -1.929335 \\ \mathrm{H} & -1.277279 & -2.999708 & -0.780853 \\ \mathrm{H} & 0.956231 & -3.344098 & 2.867384 \\ \mathrm{H} & 3.027048 & -3.485571 & 1.565744 \\ \mathrm{H} & -1.819689 & -3.598706 & 2.612055 \\ \mathrm{H} & -2.409351 & -2.641440 & 1.258631 \\ \mathrm{H} & -2.164456 & -0.043694 & 1.385021 \\ \mathrm{H} & -1.387538 & 0.650676 & 2.804455 \\ \mathrm{H} & 4.481730 & -0.644396 & -0.270718 \\ \mathrm{H} & 3.897071 & 0.294306 & -1.637967 \\ \mathrm{H} & 3.581120 & -3.957125 & -1.797603 \\ \mathrm{H} & 4.206734 & -3.221207 & -0.336147 \\ \mathrm{H} & 4.451982 & -1.873440 & -2.441743 \\ \mathrm{H} & 2.711889 & -1.703308 & -2.545380 \\ \mathrm{H} & -0.556265 & -1.607530 & 3.477311 \\ \mathrm{H} & -2.303145 & -1.442432 & 3.463872 \\ \mathrm{H} & 0.138211 & -4.413849 & -4.936954 \\ \mathrm{H} & 1.441209 & -3.223977 & -5.084837 \\ \mathrm{H} & 1.834615 & -4.961039 & -5.053119 \\ \mathrm{H} & 1.734165 & -4.927185 & -2.725586 \\ & & & \end{array}$

$\left(\boldsymbol{R}_{\mathbf{p}}\right)$-[3.3]pCp-5-monocarboxamide (Chair_syn_2), dihedral $=-137.5^{\circ}$ Total Energy: -905.615388596 a.u.

$\begin{array}{rrrr}\mathrm{C} & -0.035164 & 0.225224 & -0.145262 \\ \mathrm{C} & -0.056909 & -0.064748 & 1.222945 \\ \mathrm{C} & 1.172204 & -0.227409 & 1.864385 \\ \mathrm{C} & 2.359035 & -0.270089 & 1.139594 \\ \mathrm{C} & 2.362694 & -0.137908 & -0.248875 \\ \mathrm{C} & 1.155396 & 0.198113 & -0.869376 \\ \mathrm{C} & -1.359524 & -0.256431 & 1.975290 \\ \mathrm{C} & -1.458713 & -1.557722 & 2.798857 \\ \mathrm{C} & -1.574975 & -2.880727 & 2.011990 \\ \mathrm{C} & -0.358637 & -3.199012 & 1.173679 \\ \mathrm{C} & -0.404331 & -3.109363 & -0.215995 \\ \mathrm{C} & 0.752558 & -3.162644 & -0.995866 \\ \mathrm{C} & 2.013470 & -3.281009 & -0.388185 \\ \mathrm{C} & 2.036592 & -3.508721 & 0.990057 \\ \mathrm{C} & 0.876778 & -3.480764 & 1.759264 \\ \mathrm{C} & 3.308309 & -3.090692 & -1.155650 \\ \mathrm{C} & 3.453229 & -1.749335 & -1.908131\end{array}$




$\begin{array}{rrrr}\mathrm{C} & 3.594693 & -0.467902 & -1.059361 \\ \mathrm{C} & 0.632835 & -3.067522 & -2.489593 \\ \mathrm{O} & 1.273618 & -3.776372 & -3.254782 \\ \mathrm{~N} & -0.257878 & -2.142754 & -2.947592 \\ \mathrm{C} & -0.385024 & -1.903384 & -4.372137 \\ \mathrm{H} & 3.292217 & -0.488044 & 1.655713 \\ \mathrm{H} & 1.203527 & -0.403161 & 2.937789 \\ \mathrm{H} & -0.975987 & 0.426425 & -0.658152 \\ \mathrm{H} & 1.138650 & 0.372796 & -1.945036 \\ \mathrm{H} & -1.366396 & -2.962117 & -0.704654 \\ \mathrm{H} & 0.946688 & -3.603321 & 2.839251 \\ \mathrm{H} & 2.997652 & -3.643460 & 1.482333 \\ \mathrm{H} & -1.753413 & -3.685105 & 2.736996 \\ \mathrm{H} & -2.461111 & -2.837605 & 1.365524 \\ \mathrm{H} & -2.194419 & -0.216727 & 1.262862 \\ \mathrm{H} & -1.507756 & 0.584019 & 2.666795 \\ \mathrm{H} & 4.455454 & -0.569149 & -0.385718 \\ \mathrm{H} & 3.826430 & 0.359354 & -1.743065 \\ \mathrm{H} & 3.410622 & -3.891989 & -1.894526 \\ \mathrm{H} & 4.143111 & -3.190382 & -0.449576 \\ \mathrm{H} & 4.348528 & -1.830105 & -2.536844 \\ \mathrm{H} & 2.615668 & -1.625476 & -2.604826 \\ \mathrm{H} & -0.599798 & -1.624736 & 3.478554 \\ \mathrm{H} & -2.344643 & -1.476531 & 3.440762 \\ \mathrm{H} & -1.190440 & -1.186460 & -4.539715 \\ \mathrm{H} & 0.547566 & -1.513964 & -4.797730 \\ \mathrm{H} & -0.622850 & -2.839594 & -4.881704 \\ \mathrm{H} & -0.586937 & -1.443814 & -2.295527\end{array}$

$\left(\boldsymbol{R}_{\mathrm{p}}\right)-[3.3]$ pCp-5,14-dicarboxamide (Pseudoortho_1), dihedral $=-142.6^{\circ}, 47.6^{\circ}$ Total Energy: -1113.56152192 a.u.

$\begin{array}{rrrr}\mathrm{C} & -0.221632 & 0.356041 & 1.615079 \\ \mathrm{C} & 0.671490 & -0.632185 & 2.015450 \\ \mathrm{C} & 0.243615 & -1.961722 & 1.913226 \\ \mathrm{C} & -0.952653 & -2.279288 & 1.285817 \\ \mathrm{C} & -1.783662 & -1.291639 & 0.742519 \\ \mathrm{C} & -1.440557 & 0.041919 & 1.001817 \\ \mathrm{C} & 2.065181 & -0.285383 & 2.499845 \\ \mathrm{C} & 3.214047 & -1.010269 & 1.766459 \\ \mathrm{C} & 3.541579 & -0.510000 & 0.343710 \\ \mathrm{C} & 2.392844 & -0.714031 & -0.621279 \\ \mathrm{C} & 1.591680 & 0.328100 & -1.110539 \\ \mathrm{C} & 0.373992 & 0.031601 & -1.737303 \\ \mathrm{C} & -0.067100 & -1.277044 & -1.915082 \\ \mathrm{C} & 0.811612 & -2.308196 & -1.567974 \\ \mathrm{C} & 2.011158 & -2.024844 & -0.929757 \\ \mathrm{C} & 2.002634 & 1.769901 & -0.948414 \\ \mathrm{O} & 3.170299 & 2.136855 & -1.063166 \\ \mathrm{C} & -1.460703 & -1.574682 & -2.429175 \\ \mathrm{C} & -2.335239 & -2.422571 & -1.482481 \\ \mathrm{C} & -2.875464 & -1.709490 & -0.224211 \\ \mathrm{C} & -2.277078 & 1.205508 & 0.560700 \\ \mathrm{O} & -1.811713 & 2.151241 & -0.078802 \\ \mathrm{~N} & -3.576818 & 1.188520 & 0.933344 \\ \mathrm{C} & -4.465133 & 2.286700 & 0.598487 \\ \mathrm{~N} & 1.003236 & 2.644166 & -0.689878 \\ \mathrm{C} & 1.291542 & 4.057867 & -0.566306 \\ \mathrm{H} & 0.531380 & -3.344956 & -1.748281 \\ \mathrm{H} & 2.647131 & -2.846246 & -0.602345 \\ \mathrm{H} & -0.266623 & 0.849131 & -2.063081 \\ \mathrm{H} & -1.212473 & -3.326183 & 1.138432 \\ \mathrm{H} & 0.887870 & -2.764918 & 2.265273\end{array}$




$\begin{array}{rrrr}\mathrm{H} & 0.053381 & 1.404287 & 1.716749 \\ \mathrm{H} & 2.141506 & -0.526759 & 3.568622 \\ \mathrm{H} & 2.212149 & 0.799179 & 2.414169 \\ \mathrm{H} & 3.832158 & 0.541294 & 0.376635 \\ \mathrm{H} & 4.417634 & -1.066026 & -0.014130 \\ \mathrm{H} & -1.388332 & -2.113146 & -3.383415 \\ \mathrm{H} & -1.975185 & -0.628986 & -2.645962 \\ \mathrm{H} & -3.461794 & -0.842285 & -0.544457 \\ \mathrm{H} & -3.572585 & -2.394905 & 0.276974 \\ \mathrm{H} & -1.780755 & -3.321446 & -1.184451 \\ \mathrm{H} & -3.203915 & -2.774918 & -2.052331 \\ \mathrm{H} & 3.003287 & -2.087591 & 1.739519 \\ \mathrm{H} & 4.120723 & -0.893175 & 2.372535 \\ \mathrm{H} & -3.891393 & 0.452611 & 1.549873 \\ \mathrm{H} & -5.479870 & 2.022605 & 0.898365 \\ \mathrm{H} & -4.160845 & 3.206443 & 1.107868 \\ \mathrm{H} & -4.439470 & 2.468727 & -0.478672 \\ \mathrm{H} & 0.047987 & 2.327804 & -0.535723 \\ \mathrm{H} & 1.712975 & 4.450227 & -1.497122 \\ \mathrm{H} & 0.359845 & 4.578697 & -0.339096 \\ \mathrm{H} & 2.018673 & 4.239216 & 0.231985\end{array}$

$\left(\boldsymbol{R}_{\mathrm{p}}\right)$-[3.3]pCp-5,14-dicarboxamide (Pseudoortho_2), dihedral $=-140.5^{\circ}, 50.6^{\circ}$ Total Energy: -1113.56340448 a.u.

$\begin{array}{rrrr}\mathrm{C} & 1.609179 & 0.012996 & -1.144841 \\ \mathrm{C} & 2.209379 & -1.095398 & -0.524176 \\ \mathrm{C} & 1.636440 & -2.348197 & -0.747471 \\ \mathrm{C} & 0.438552 & -2.491408 & -1.440825 \\ \mathrm{C} & -0.245042 & -1.372045 & -1.917300 \\ \mathrm{C} & 0.392059 & -0.134371 & -1.814211 \\ \mathrm{C} & 3.343430 & -0.946626 & 0.467774 \\ \mathrm{C} & 2.970055 & -0.115168 & 1.713182 \\ \mathrm{C} & 1.926608 & -0.728268 & 2.670348 \\ \mathrm{C} & 0.519659 & -0.822178 & 2.117376 \\ \mathrm{C} & -0.141519 & -2.048976 & 2.011053 \\ \mathrm{C} & -1.346756 & -2.158272 & 1.327372 \\ \mathrm{C} & -1.953477 & -1.048880 & 0.732530 \\ \mathrm{C} & -1.378366 & 0.206197 & 0.988557 \\ \mathrm{C} & -0.151752 & 0.308105 & 1.651176 \\ \mathrm{C} & -3.075677 & -1.265250 & -0.267066 \\ \mathrm{C} & -2.667968 & -2.120813 & -1.486705 \\ \mathrm{C} & -1.653009 & -1.491005 & -2.462558 \\ \mathrm{C} & -2.004540 & 1.489865 & 0.530248 \\ \mathrm{~N} & -3.284646 & 1.694961 & 0.914416 \\ \mathrm{C} & -3.982299 & 2.919634 & 0.567349 \\ \mathrm{C} & 2.250611 & 1.372508 & -1.052074 \\ \mathrm{~N} & 1.405939 & 2.406267 & -0.840087 \\ \mathrm{C} & 1.922394 & 3.755462 & -0.742105 \\ \mathrm{O} & 3.464560 & 1.530137 & -1.162296 \\ \mathrm{O} & -1.396678 & 2.338240 & -0.125104 \\ \mathrm{H} & -0.000622 & -3.482086 & -1.548109 \\ \mathrm{H} & 2.104963 & -3.226255 & -0.306591 \\ \mathrm{H} & -0.090629 & 0.746375 & -2.234920 \\ \mathrm{H} & -1.786331 & -3.143522 & 1.181810 \\ \mathrm{H} & 0.332507 & -2.946136 & 2.404186 \\ \mathrm{H} & 0.300136 & 1.293765 & 1.756514 \\ \mathrm{H} & 2.256997 & -1.731131 & 2.970923 \\ \mathrm{H} & 1.914448 & -0.120021 & 3.584566 \\ \mathrm{H} & 4.198787 & -0.464941 & -0.013157 \\ \mathrm{H} & 3.660532 & -1.946842 & 0.790950 \\ \mathrm{H} & -1.644443 & -2.105311 & -3.372626 \\ \mathrm{H} & -2.009636 & -0.496886 & -2.762588 \\ & & & \end{array}$




$\begin{array}{rrrr}\mathrm{H} & -3.455765 & -0.305533 & -0.631987 \\ \mathrm{H} & -3.917415 & -1.769043 & 0.227527 \\ \mathrm{H} & -2.284572 & -3.089514 & -1.142518 \\ \mathrm{H} & -3.583900 & -2.338677 & -2.049758 \\ \mathrm{H} & 3.889108 & 0.046430 & 2.289407 \\ \mathrm{H} & 2.636637 & 0.882511 & 1.401048 \\ \mathrm{H} & -3.716582 & 1.022838 & 1.532661 \\ \mathrm{H} & -5.012379 & 2.848314 & 0.918215 \\ \mathrm{H} & -3.498765 & 3.786797 & 1.027386 \\ \mathrm{H} & -3.975367 & 3.059828 & -0.516598 \\ \mathrm{H} & 0.420209 & 2.242813 & -0.639060 \\ \mathrm{H} & 1.084206 & 4.432545 & -0.569279 \\ \mathrm{H} & 2.640902 & 3.842792 & 0.080533 \\ \mathrm{H} & 2.437110 & 4.037806 & -1.665454\end{array}$

$\left(\boldsymbol{R}_{\mathbf{p}}\right)$-[3.3]pCp-5,14-dicarboxamide (Pseudoortho_3), dihedral $=-138.9^{\circ}, 45.8^{\circ}$ Total Energy: -1113.56232158 a.u.

\begin{tabular}{|c|c|c|c|}
\hline $\begin{array}{l}\text { C } \\
\text { C } \\
\text { C } \\
\text { C } \\
\text { C } \\
\text { C } \\
\text { C } \\
C \\
C\end{array}$ & 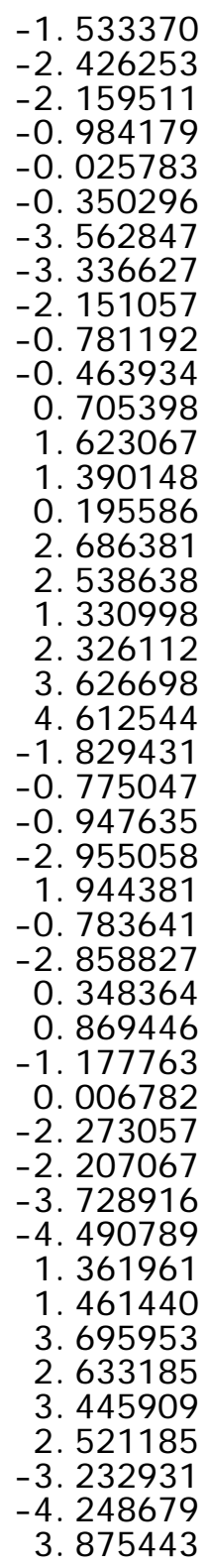 & 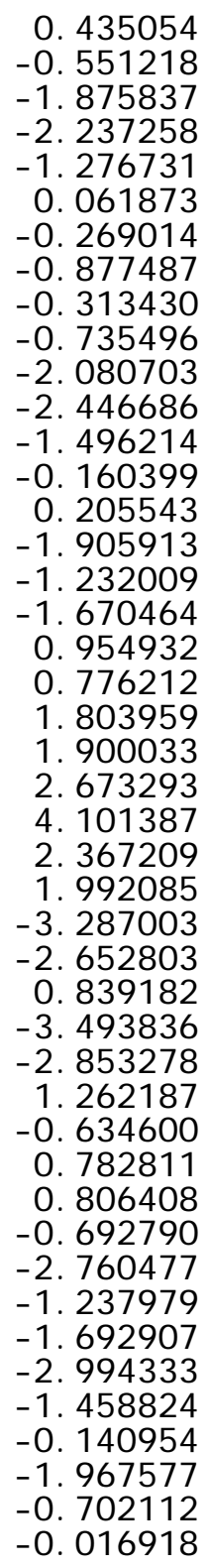 & $\begin{array}{r}1.119619 \\
0.666214 \\
1.019432 \\
1.669862 \\
1.993344 \\
1.765039 \\
-0.294987 \\
-1.695143 \\
-2.506343 \\
-2.014816 \\
-1.810978 \\
-1.153518 \\
-0.699298 \\
-1.068213 \\
-1.693573 \\
0.304023 \\
1.686124 \\
2.538855 \\
-0.712678 \\
-1.034175 \\
-0.752906 \\
0.918153 \\
0.574889 \\
0.407499 \\
1.078503 \\
-0.165832 \\
1.877089 \\
0.713460 \\
2.073406 \\
-0.907447 \\
-2.089904 \\
-1.874905 \\
-3.549576 \\
-2.509120 \\
-0.382784 \\
0.109798 \\
2.667168 \\
3.539542 \\
-0.065224 \\
0.433369 \\
2.259817 \\
1.567176 \\
-1.614384 \\
-2.278705 \\
-1.607962\end{array}$ \\
\hline
\end{tabular}




$\begin{array}{rrrr}\mathrm{H} & 5.600483 & 1.424533 & -1.016820 \\ \mathrm{H} & 4.405082 & 2.714802 & -1.323045 \\ \mathrm{H} & 4.591655 & 2.054071 & 0.310700 \\ \mathrm{H} & 0.138194 & 2.269466 & 0.373057 \\ \mathrm{H} & 0.016035 & 4.532324 & 0.130638 \\ \mathrm{H} & -1.684984 & 4.317470 & -0.372796 \\ \mathrm{H} & -1.301149 & 4.558735 & 1.336827\end{array}$

$\left(\boldsymbol{R}_{\mathbf{p}}\right)$-[3.3]pCp-5,14-dicarboxamide (Pseudoortho_4), dihedral $=-138.2^{\circ}, 47.1^{\circ}$

Total Energy: -1113.56481113 a.u.

$\begin{array}{rrrr}\mathrm{C} & 1.545165 & 0.155910 & -1.178010 \\ \mathrm{C} & 2.235686 & -0.944468 & -0.633742 \\ \mathrm{C} & 1.748737 & -2.216281 & -0.924613 \\ \mathrm{C} & 0.550539 & -2.402034 & -1.612866 \\ \mathrm{C} & -0.209100 & -1.310949 & -2.024569 \\ \mathrm{C} & 0.339043 & -0.035277 & -1.850083 \\ \mathrm{C} & 3.390291 & -0.773089 & 0.332297 \\ \mathrm{C} & 3.030682 & -0.009837 & 1.624687 \\ \mathrm{C} & 2.088325 & -0.726091 & 2.614499 \\ \mathrm{C} & 0.680830 & -0.934434 & 2.100529 \\ \mathrm{C} & 0.159123 & -2.211209 & 1.898147 \\ \mathrm{C} & -1.037915 & -2.396075 & 1.210867 \\ \mathrm{C} & -1.778741 & -1.319768 & 0.722595 \\ \mathrm{C} & -1.336866 & -0.030525 & 1.076916 \\ \mathrm{C} & -0.118740 & 0.150428 & 1.731824 \\ \mathrm{C} & -2.900314 & -1.569902 & -0.272448 \\ \mathrm{C} & -2.711970 & -0.895152 & -1.649372 \\ \mathrm{C} & -1.613357 & -1.482382 & -2.558737 \\ \mathrm{C} & -2.090229 & 1.213749 & 0.712413 \\ \mathrm{~N} & -3.403365 & 1.232254 & 1.033082 \\ \mathrm{C} & -4.226678 & 2.392531 & 0.745734 \\ \mathrm{C} & 2.097817 & 1.547622 & -1.016369 \\ \mathrm{~N} & 1.195017 & 2.502372 & -0.700583 \\ \mathrm{C} & 1.624797 & 3.872681 & -0.513926 \\ \mathrm{O} & 3.292663 & 1.794852 & -1.161565 \\ \mathrm{O} & -1.557181 & 2.180999 & 0.165185 \\ \mathrm{H} & 0.168736 & -3.410230 & -1.764777 \\ \mathrm{H} & 2.280378 & -3.084239 & -0.538648 \\ \mathrm{H} & -0.203354 & 0.834835 & -2.219280 \\ \mathrm{H} & -1.358598 & -3.407349 & 0.968936 \\ \mathrm{H} & 0.740905 & -3.080186 & 2.199010 \\ \mathrm{H} & 0.228986 & 1.168239 & 1.905454 \\ \mathrm{H} & 2.517654 & -1.699805 & 2.884238 \\ \mathrm{H} & 2.057335 & -0.132473 & 3.537610 \\ \mathrm{H} & 4.203257 & -0.231899 & -0.159529 \\ \mathrm{H} & 3.768815 & -1.767781 & 0.602792 \\ \mathrm{H} & -1.812006 & -2.549879 & -2.720603 \\ \mathrm{H} & -1.700719 & -0.996377 & -3.539114 \\ \mathrm{H} & -3.867651 & -1.243539 & 0.127197 \\ \mathrm{H} & -2.987309 & -2.654043 & -0.419934 \\ \mathrm{H} & -3.665496 & -0.975215 & -2.186128 \\ \mathrm{H} & -2.528248 & 0.178587 & -1.516515 \\ \mathrm{H} & 3.967610 & 0.198123 & 2.155395 \\ \mathrm{H} & 2.610554 & 0.971353 & 1.370023 \\ \mathrm{H} & -3.769072 & 0.485220 & 1.606056 \\ \mathrm{H} & -5.262789 & 2.158022 & 0.992803 \\ \mathrm{H} & -3.899733 & 3.261935 & 1.324649 \\ \mathrm{H} & -4.154222 & 2.641426 & -0.315946 \\ \mathrm{H} & 0.235179 & 2.253606 & -0.465355 \\ \mathrm{H} & 0.755732 & 4.469792 & -0.233341 \\ \mathrm{H} & 2.386837 & 3.940635 & 0.270457 \\ \mathrm{H} & 2.059044 & 4.269362 & -1.436530 \\ & & & \end{array}$


[3.3]pCp-5,18-dicarboxamide (Pseudopara_1), dihedral $=-139.6^{\circ},-46.1^{\circ}$ Total Energy: -1113.55180581 a.u.

$\begin{array}{rrrr}\mathrm{C} & 2.038748 & -0.482023 & 0.904851 \\ \mathrm{C} & 1.587225 & 0.743452 & 1.417125 \\ \mathrm{C} & 0.345159 & 0.751234 & 2.062669 \\ \mathrm{C} & -0.479095 & -0.365762 & 2.086827 \\ \mathrm{C} & -0.084787 & -1.551879 & 1.459345 \\ \mathrm{C} & 1.201489 & -1.605410 & 0.932540 \\ \mathrm{C} & 2.310431 & 2.060430 & 1.208460 \\ \mathrm{C} & 1.505241 & 3.097206 & 0.398626 \\ \mathrm{C} & 1.253071 & 2.768047 & -1.088292 \\ \mathrm{C} & 0.285094 & 1.626579 & -1.312521 \\ \mathrm{C} & 0.664470 & 0.458540 & -1.973707 \\ \mathrm{C} & -0.151573 & -0.671089 & -1.950782 \\ \mathrm{C} & -1.381171 & -0.678174 & -1.288879 \\ \mathrm{C} & -1.833698 & 0.550820 & -0.774432 \\ \mathrm{C} & -1.002920 & 1.669766 & -0.777926 \\ \mathrm{C} & -2.085704 & -1.999518 & -1.028014 \\ \mathrm{C} & -2.309692 & -2.340857 & 0.461557 \\ \mathrm{C} & -1.054575 & -2.699091 & 1.282126 \\ \mathrm{C} & -3.156221 & 0.716453 & -0.080103 \\ \mathrm{~N} & -4.244182 & 0.254755 & -0.751317 \\ \mathrm{C} & -5.572084 & 0.401205 & -0.185433 \\ \mathrm{C} & 3.393926 & -0.609190 & 0.268982 \\ \mathrm{~N} & 3.437177 & -1.369517 & -0.863771 \\ \mathrm{C} & 4.687409 & -1.546639 & -1.575900 \\ \mathrm{O} & 4.408498 & -0.098679 & 0.726827 \\ \mathrm{O} & -3.256084 & 1.241952 & 1.022416 \\ \mathrm{H} & 0.207983 & -1.599875 & -2.392889 \\ \mathrm{H} & 1.644919 & 0.407608 & -2.444670 \\ \mathrm{H} & -1.359117 & 2.568201 & -0.276906 \\ \mathrm{H} & 0.868680 & 3.675018 & -1.573285 \\ \mathrm{H} & 2.209149 & 2.533531 & -1.574170 \\ \mathrm{H} & 0.543693 & 3.289866 & 0.891431 \\ \mathrm{H} & 2.060006 & 4.042649 & 0.436541 \\ \mathrm{H} & 2.528133 & 2.500785 & 2.190203 \\ \mathrm{H} & 3.278602 & 1.892677 & 0.732134 \\ \mathrm{H} & -0.022970 & 1.679903 & 2.494668 \\ \mathrm{H} & -1.467791 & -0.283559 & 2.534267 \\ \mathrm{H} & 1.555586 & -2.540790 & 0.501326 \\ \mathrm{H} & -1.391673 & -3.056215 & 2.263878 \\ \mathrm{H} & -0.538106 & -3.541675 & 0.803823 \\ \mathrm{H} & -2.838275 & -1.518231 & 0.958185 \\ \mathrm{H} & -2.987505 & -3.202763 & 0.500057 \\ \mathrm{H} & -1.489114 & -2.799287 & -1.486100 \\ \mathrm{H} & -3.062293 & -2.030622 & -1.525267 \\ \mathrm{H} & -4.138485 & -0.007984 & -1.720557 \\ \mathrm{H} & -6.297684 & -0.038514 & -0.871008 \\ \mathrm{H} & -5.625193 & -0.114982 & 0.777059 \\ \mathrm{H} & -5.815460 & 1.455407 & -0.019174 \\ \mathrm{H} & 2.566978 & -1.626222 & -1.306970 \\ \mathrm{H} & 4.540834 & -2.277821 & -2.372556 \\ \mathrm{H} & 5.042735 & -0.603110 & -2.005233 \\ \mathrm{H} & 5.451638 & -1.911436 & -0.885831 \\ & & & \end{array}$

[3.3]pCp-5,18-dicarboxamide (Pseudopara_2), dihedral $=-140.4^{\circ},-45.9^{\circ}$ Total Energy: -1113.55389537 a.u.
C
0. 275274
0.546235
2. 100314 


$\begin{array}{rrrr}\mathrm{C} & 1.541097 & 0.571720 & 1.511851 \\ \mathrm{C} & 1.993638 & -0.611829 & 0.904827 \\ \mathrm{C} & 1.143053 & -1.715548 & 0.793513 \\ \mathrm{C} & -0.163145 & -1.687951 & 1.279532 \\ \mathrm{C} & -0.563768 & -0.558387 & 1.995177 \\ \mathrm{C} & 2.311937 & 1.874153 & 1.431817 \\ \mathrm{C} & 2.502047 & 2.419865 & 0.000032 \\ \mathrm{C} & 1.232990 & 2.904963 & -0.730945 \\ \mathrm{C} & 0.250279 & 1.806586 & -1.070256 \\ \mathrm{C} & -1.031483 & 1.788692 & -0.528400 \\ \mathrm{C} & -1.878181 & 0.687347 & -0.671602 \\ \mathrm{C} & -1.436533 & -0.469663 & -1.333413 \\ \mathrm{C} & -0.198845 & -0.393626 & -1.982204 \\ \mathrm{C} & 0.623285 & 0.722739 & -1.871115 \\ \mathrm{C} & -2.154265 & -1.807153 & -1.249066 \\ \mathrm{C} & -2.389371 & -2.339793 & 0.181631 \\ \mathrm{C} & -1.140850 & -2.797885 & 0.962248 \\ \mathrm{C} & -3.201055 & 0.780701 & 0.035049 \\ \mathrm{~N} & -4.291388 & 0.419583 & -0.692380 \\ \mathrm{C} & -5.620891 & 0.516035 & -0.119555 \\ \mathrm{C} & 3.364894 & -0.675129 & 0.298287 \\ \mathrm{~N} & 3.436123 & -1.293531 & -0.916301 \\ \mathrm{C} & 4.695205 & -1.343814 & -1.633541 \\ \mathrm{O} & 4.362800 & -0.210094 & 0.833620 \\ \mathrm{O} & -3.298925 & 1.165214 & 1.194580 \\ \mathrm{H} & 0.152063 & -1.263941 & -2.535863 \\ \mathrm{H} & 1.600835 & 0.721306 & -2.352150 \\ \mathrm{H} & -1.371824 & 2.616399 & 0.090904 \\ \mathrm{H} & 0.727351 & 3.659996 & -0.115643 \\ \mathrm{H} & 1.548740 & 3.412652 & -1.652131 \\ \mathrm{H} & 3.192305 & 3.269833 & 0.062266 \\ \mathrm{H} & 3.012886 & 1.669151 & -0.616816 \\ \mathrm{H} & 1.779838 & 2.628075 & 2.026165 \\ \mathrm{H} & 3.303369 & 1.745244 & 1.874734 \\ \mathrm{H} & -0.101626 & 1.444919 & 2.584224 \\ \mathrm{H} & -1.570554 & -0.492611 & 2.402458 \\ \mathrm{H} & 1.498875 & -2.611176 & 0.285399 \\ \mathrm{H} & -1.486458 & -3.266841 & 1.892792 \\ \mathrm{H} & -0.625855 & -3.581826 & 0.391460 \\ \mathrm{H} & -2.929548 & -1.590817 & 0.773603 \\ \mathrm{H} & -3.063435 & -3.201747 & 0.101358 \\ \mathrm{H} & -1.563365 & -2.545567 & -1.806708 \\ \mathrm{H} & -3.128940 & -1.762727 & -1.749017 \\ \mathrm{H} & -4.183500 & 0.288481 & -1.687974 \\ \mathrm{H} & -5.871579 & 1.552021 & 0.130555 \\ \mathrm{H} & -6.343499 & 0.127751 & -0.838571 \\ \mathrm{H} & -5.670055 & -0.076347 & 0.797687 \\ \mathrm{H} & 2.573681 & -1.491746 & -1.403459 \\ \mathrm{H} & 4.562045 & -1.932547 & -2.542643 \\ \mathrm{H} & 5.044583 & -0.338332 & -1.895476 \\ \mathrm{H} & 5.457630 & -1.812489 & -1.007078\end{array}$

[3.3]pCp-5,18-dicarboxamide (Pseudopara_3), dihedral $=-141.3^{\circ},-48.2^{\circ}$ Total Energy: -1113.55054520 a.u.

$\begin{array}{rrrr}\mathrm{C} & -0.897085 & 1.388042 & -0.944822 \\ \mathrm{C} & 0.344929 & 1.192287 & -1.543891 \\ \mathrm{C} & 0.589535 & -0.066159 & -2.106485 \\ \mathrm{C} & -0.295254 & -1.121904 & -1.911169 \\ \mathrm{C} & -1.476438 & -0.960228 & -1.177146 \\ \mathrm{C} & -1.810369 & 0.343346 & -0.783655 \\ \mathrm{C} & 1.399966 & 2.283164 & -1.566783 \\ \mathrm{C} & 1.591423 & 3.064940 & -0.251128\end{array}$




$\begin{array}{rrrr}\mathrm{C} & 2.326893 & 2.325806 & 0.887161 \\ \mathrm{C} & 1.579431 & 1.096121 & 1.356503 \\ \mathrm{C} & 1.951534 & -0.212764 & 1.006451 \\ \mathrm{C} & 1.054954 & -1.268121 & 1.203541 \\ \mathrm{C} & -0.216468 & -1.069039 & 1.739526 \\ \mathrm{C} & -0.521718 & 0.207467 & 2.215756 \\ \mathrm{C} & 0.364574 & 1.262811 & 2.025781 \\ \mathrm{C} & 3.284279 & -0.506498 & 0.375938 \\ \mathrm{O} & 4.330385 & 0.031082 & 0.717544 \\ \mathrm{C} & -1.237914 & -2.190117 & 1.762907 \\ \mathrm{C} & -1.435651 & -2.938235 & 0.428168 \\ \mathrm{C} & -2.210488 & -2.188125 & -0.677753 \\ \mathrm{C} & -3.107767 & 0.699474 & -0.108853 \\ \mathrm{O} & -3.160360 & 1.341229 & 0.933386 \\ \mathrm{~N} & -4.234226 & 0.304639 & -0.759751 \\ \mathrm{C} & -5.544278 & 0.657233 & -0.246015 \\ \mathrm{~N} & 3.266099 & -1.458019 & -0.602923 \\ \mathrm{C} & 4.482260 & -1.791628 & -1.317452 \\ \mathrm{H} & -0.038343 & -2.115110 & -2.280092 \\ \mathrm{H} & 1.518769 & -0.231011 & -2.650098 \\ \mathrm{H} & -1.163735 & 2.355509 & -0.525832 \\ \mathrm{H} & 1.145593 & 3.005780 & -2.354222 \\ \mathrm{H} & 2.360110 & 1.836935 & -1.860697 \\ \mathrm{H} & 0.618552 & 3.419826 & 0.111025 \\ \mathrm{H} & 2.172078 & 3.965306 & -0.485728 \\ \mathrm{H} & 2.433215 & 3.025357 & 1.725672 \\ \mathrm{H} & 3.336846 & 2.063629 & 0.566821 \\ \mathrm{H} & 0.061956 & 2.264206 & 2.327744 \\ \mathrm{H} & -1.494862 & 0.401624 & 2.658264 \\ \mathrm{H} & 1.355217 & -2.272157 & 0.908448 \\ \mathrm{H} & -2.202264 & -1.781761 & 2.093498 \\ \mathrm{H} & -0.944003 & -2.932231 & 2.517564 \\ \mathrm{H} & -1.988190 & -3.861691 & 0.641098 \\ \mathrm{H} & -0.460790 & -3.251875 & 0.033028 \\ \mathrm{H} & -2.371573 & -2.887808 & -1.508432 \\ \mathrm{H} & -3.198334 & -1.921157 & -0.289375 \\ \mathrm{H} & -4.144439 & -0.102129 & -1.679939 \\ \mathrm{H} & -6.304859 & 0.249270 & -0.912962 \\ \mathrm{H} & -5.680476 & 0.241108 & 0.755849 \\ \mathrm{H} & -5.658612 & 1.743826 & -0.180891 \\ \mathrm{H} & 2.371982 & -1.721347 & -0.992464 \\ \mathrm{H} & 4.295422 & -2.662834 & -1.947879 \\ \mathrm{H} & 4.828086 & -0.957014 & -1.938714 \\ \mathrm{H} & 5.271269 & -2.026332 & -0.599872 \\ & & & \\ & -5361 & \end{array}$

[3.3]pCp-5,18-dicarboxamide (Pseudopara_4), dihedral $=-140.2^{\circ},-50.1^{\circ}$ Total Energy: -1113.55327708 a.u.

$\begin{array}{lrrr}\mathrm{C} & 1.135227 & -1.713074 & -0.620249 \\ \mathrm{C} & -0.129196 & -1.706941 & -1.196720 \\ \mathrm{C} & -0.502882 & -0.565032 & -1.918653 \\ \mathrm{C} & 0.300421 & 0.567401 & -1.921579 \\ \mathrm{C} & 1.517708 & 0.609402 & -1.227025 \\ \mathrm{C} & 1.963195 & -0.584975 & -0.647476 \\ \mathrm{C} & -1.095763 & -2.853634 & -0.994320 \\ \mathrm{C} & -2.398260 & -2.475779 & -0.259276 \\ \mathrm{C} & -2.267426 & -2.085735 & 1.227874 \\ \mathrm{C} & -1.528455 & -0.784146 & 1.459902 \\ \mathrm{C} & -2.000290 & 0.446287 & 0.963791 \\ \mathrm{C} & -1.175831 & 1.571150 & 0.976122 \\ \mathrm{C} & 0.132720 & 1.516629 & 1.463814 \\ \mathrm{C} & 0.548181 & 0.333943 & 2.069604 \\ \mathrm{C} & -0.274869 & -0.791621 & 2.066353\end{array}$




$\begin{array}{rrrr}\mathrm{C} & -3.366315 & 0.542920 & 0.349437 \\ \mathrm{O} & -4.360710 & 0.008836 & 0.822835 \\ \mathrm{C} & 1.083748 & 2.671705 & 1.238596 \\ \mathrm{C} & 1.354983 & 2.980160 & -0.248775 \\ \mathrm{C} & 2.203447 & 1.948650 & -1.022633 \\ \mathrm{C} & 3.279591 & -0.713153 & 0.068326 \\ \mathrm{O} & 3.366842 & -1.127467 & 1.218194 \\ \mathrm{~N} & 4.379605 & -0.372941 & -0.653899 \\ \mathrm{C} & 5.708045 & -0.508989 & -0.087037 \\ \mathrm{~N} & -3.435173 & 1.280492 & -0.797438 \\ \mathrm{C} & -4.688020 & 1.386345 & -1.519417 \\ \mathrm{H} & -0.044696 & 1.467750 & -2.429948 \\ \mathrm{H} & -1.462102 & -0.548239 & -2.435289 \\ \mathrm{H} & 1.475422 & -2.585399 & -0.065436 \\ \mathrm{H} & -0.588674 & -3.655920 & -0.443374 \\ \mathrm{H} & -1.371882 & -3.272976 & -1.971138 \\ \mathrm{H} & -3.071406 & -3.339831 & -0.315600 \\ \mathrm{H} & -2.904201 & -1.669370 & -0.806070 \\ \mathrm{H} & -1.737246 & -2.886599 & 1.758803 \\ \mathrm{H} & -3.274970 & -2.022586 & 1.648094 \\ \mathrm{H} & 0.115308 & -1.726020 & 2.464584 \\ \mathrm{H} & 1.556881 & 0.248482 & 2.466614 \\ \mathrm{H} & -1.551682 & 2.507941 & 0.564931 \\ \mathrm{H} & 2.035340 & 2.462106 & 1.743819 \\ \mathrm{H} & 0.677638 & 3.582113 & 1.698710 \\ \mathrm{H} & 1.883471 & 3.940051 & -0.302595 \\ \mathrm{H} & 0.397684 & 3.130401 & -0.765460 \\ \mathrm{H} & 2.448579 & 2.385792 & -2.000579 \\ \mathrm{H} & 3.152382 & 1.814831 & -0.493818 \\ \mathrm{H} & 4.267514 & -0.155530 & -1.633758 \\ \mathrm{H} & 6.437414 & -0.130124 & -0.804160 \\ \mathrm{H} & 5.778807 & 0.066048 & 0.839955 \\ \mathrm{H} & 5.931309 & -1.555288 & 0.144216 \\ \mathrm{H} & -2.570767 & 1.534235 & -1.254671 \\ \mathrm{H} & -4.571243 & 2.108484 & -2.329083 \\ \mathrm{H} & -4.997087 & 0.418652 & -1.931888 \\ \mathrm{H} & -5.471625 & 1.728096 & -0.839685\end{array}$

\section{$\left(R_{p}\right)$-[3.3]pCp-5, 8, 14, 17-tetracarboxamide (Anti_chair)}

Dihedral $=-132.4^{\circ}, 53.4^{\circ}, 46.8^{\circ},-138.9^{\circ}$

Total Energy: -1529.44961429 a.u.

$\begin{array}{rrrr}\mathrm{C} & -0.963868 & -0.822870 & 1.829598 \\ \mathrm{C} & 0.262137 & -1.467984 & 1.590082 \\ \mathrm{C} & 1.392621 & -0.661925 & 1.468458 \\ \mathrm{C} & 1.316078 & 0.732730 & 1.477394 \\ \mathrm{C} & 0.071919 & 1.374938 & 1.543648 \\ \mathrm{C} & -1.046677 & 0.568980 & 1.773554 \\ \mathrm{C} & 0.385950 & -2.964849 & 1.383073 \\ \mathrm{C} & 0.944070 & -3.370498 & 0.003756 \\ \mathrm{C} & 0.053369 & -3.058438 & -1.218756 \\ \mathrm{C} & -0.058600 & -1.583017 & -1.539746 \\ \mathrm{C} & 1.066385 & -0.788112 & -1.820510 \\ \mathrm{C} & 0.956426 & 0.602229 & -1.817294 \\ \mathrm{C} & -0.261560 & 1.256099 & -1.615319 \\ \mathrm{C} & -1.409044 & 0.451010 & -1.543281 \\ \mathrm{C} & -1.290811 & -0.938292 & -1.465056 \\ \mathrm{C} & 2.402565 & -1.433441 & -2.086749 \\ \mathrm{O} & 2.503021 & -2.430765 & -2.796167 \\ \mathrm{C} & -2.794512 & 1.015761 & -1.464316 \\ \mathrm{O} & -3.615802 & 0.661756 & -0.614824 \\ \mathrm{C} & -0.277475 & 2.751932 & -1.347792\end{array}$




$\begin{array}{rrrr}\mathrm{C} & -0.929831 & 3.162142 & -0.009851 \\ \mathrm{C} & -0.115869 & 2.855206 & 1.264643 \\ \mathrm{C} & 2.609886 & 1.482737 & 1.364800 \\ \mathrm{O} & 3.423322 & 1.285725 & 0.459364 \\ \mathrm{C} & -2.199643 & -1.622634 & 2.161097 \\ \mathrm{O} & -2.176148 & -2.519657 & 2.999717 \\ \mathrm{~N} & -3.320750 & -1.271405 & 1.498309 \\ \mathrm{C} & -4.571437 & -1.948597 & 1.775001 \\ \mathrm{~N} & 2.862357 & 2.379072 & 2.343885 \\ \mathrm{C} & 4.101959 & 3.134546 & 2.367692 \\ \mathrm{~N} & -3.114331 & 1.940757 & -2.395495 \\ \mathrm{C} & -4.436610 & 2.539300 & -2.428162 \\ \mathrm{~N} & 3.467773 & -0.844236 & -1.503031 \\ \mathrm{C} & 4.793297 & -1.397723 & -1.691377 \\ \mathrm{H} & 2.368328 & -1.124930 & 1.325192 \\ \mathrm{H} & -2.022977 & 1.040555 & 1.882320 \\ \mathrm{H} & 1.858911 & 1.201022 & -1.928219 \\ \mathrm{H} & -2.191614 & -1.524816 & -1.291100 \\ \mathrm{H} & -0.636064 & 3.324006 & 2.110560 \\ \mathrm{H} & 0.855417 & 3.353494 & 1.179491 \\ \mathrm{H} & -1.087647 & 4.247418 & -0.037659 \\ \mathrm{H} & -1.926634 & 2.714392 & 0.080300 \\ \mathrm{H} & -0.791835 & 3.293689 & -2.150399 \\ \mathrm{H} & 0.760447 & 3.108704 & -1.359225 \\ \mathrm{H} & 0.467766 & -3.587490 & -2.081745 \\ \mathrm{H} & -0.953836 & -3.453957 & -1.034646 \\ \mathrm{H} & -0.583004 & -3.443041 & 1.542226 \\ \mathrm{H} & 1.052430 & -3.373577 & 2.153447 \\ \mathrm{H} & 1.099741 & -4.455581 & 0.024080 \\ \mathrm{H} & 1.938893 & -2.932707 & -0.145055 \\ \mathrm{H} & -3.296264 & -0.560983 & 0.766805 \\ \mathrm{H} & -2.473975 & 2.092282 & -3.161956 \\ \mathrm{H} & 2.219714 & 2.436164 & 3.121545 \\ \mathrm{H} & 3.341712 & -0.075857 & -0.844776 \\ \mathrm{H} & 4.831029 & -2.439476 & -1.354926 \\ \mathrm{H} & 5.500436 & -0.799944 & -1.114351 \\ \mathrm{H} & 5.073371 & -1.377382 & -2.748801 \\ \mathrm{H} & 4.249589 & 3.632243 & 1.406272 \\ \mathrm{H} & 4.958392 & 2.477478 & 2.548490 \\ \mathrm{H} & 4.042649 & 3.882092 & 3.159539 \\ \mathrm{H} & -4.470330 & -3.027048 & 1.617691 \\ \mathrm{H} & -5.333104 & -1.549333 & 1.103555 \\ \mathrm{H} & -4.877997 & -1.788127 & 2.813488 \\ \mathrm{H} & -4.655775 & 3.002142 & -1.462433 \\ \mathrm{H} & -5.204552 & 1.786175 & -2.629413 \\ \mathrm{H} & -4.458071 & 3.301217 & -3.208225 \\ & & & \end{array}$

$\left(R_{\mathrm{p}}\right)-[3.3] \mathrm{pCp}-5,8,14,17$-tetracarboxamide (Anti_boat) Dihedral $=-135.5^{\circ}, 51.5^{\circ}, 49.3^{\circ},-138.9^{\circ}$

Total Energy: -1529.44996641 a.u.

$\begin{array}{rrrr}C & -0.965605 & -0.640756 & 1.855188 \\ C & 0.205092 & -1.383227 & 1.603314 \\ C & 1.389877 & -0.670092 & 1.461137 \\ C & 1.421998 & 0.729551 & 1.451112 \\ C & 0.233432 & 1.463863 & 1.522030 \\ C & -0.942142 & 0.749036 & 1.783115 \\ C & 0.189976 & -2.887830 & 1.421666 \\ C & -0.732182 & -3.398303 & 0.292800 \\ C & -0.250354 & -3.150377 & -1.151194 \\ C & -0.176893 & -1.680633 & -1.507970 \\ C & 1.023272 & -1.002728 & -1.768182\end{array}$




$\begin{array}{rrrr}\mathrm{C} & 1.042430 & 0.395496 & -1.790837 \\ \mathrm{C} & -0.106747 & 1.166229 & -1.623115 \\ \mathrm{C} & -1.328561 & 0.474734 & -1.550605 \\ \mathrm{C} & -1.345128 & -0.915955 & -1.462621 \\ \mathrm{C} & 2.310768 & -1.757464 & -1.991256 \\ \mathrm{O} & 2.355109 & -2.771595 & -2.682476 \\ \mathrm{C} & -2.653671 & 1.172474 & -1.501102 \\ \mathrm{O} & -3.503303 & 0.938580 & -0.638224 \\ \mathrm{C} & 0.016147 & 2.667962 & -1.418852 \\ \mathrm{C} & -0.628013 & 3.217298 & -0.126838 \\ \mathrm{C} & 0.140538 & 2.938567 & 1.181666 \\ \mathrm{C} & 2.767786 & 1.369414 & 1.282469 \\ \mathrm{O} & 3.541364 & 1.066266 & 0.370815 \\ \mathrm{C} & -2.248603 & -1.351120 & 2.204313 \\ \mathrm{O} & -2.267636 & -2.277044 & 3.010681 \\ \mathrm{~N} & -3.358644 & -0.898933 & 1.584454 \\ \mathrm{C} & -4.635544 & -1.535660 & 1.836137 \\ \mathrm{~N} & 3.113136 & 2.285010 & 2.213874 \\ \mathrm{C} & 4.409285 & 2.938243 & 2.178231 \\ \mathrm{~N} & -2.883188 & 2.082501 & -2.472940 \\ \mathrm{C} & -4.142098 & 2.802748 & -2.538245 \\ \mathrm{~N} & 3.407565 & -1.231678 & -1.403789 \\ \mathrm{C} & 4.703059 & -1.849349 & -1.601209 \\ \mathrm{H} & 2.321633 & -1.210704 & 1.303433 \\ \mathrm{H} & -1.877314 & 1.296470 & 1.898361 \\ \mathrm{H} & 1.998133 & 0.905236 & -1.895384 \\ \mathrm{H} & -2.303188 & -1.411530 & -1.308740 \\ \mathrm{H} & -0.372841 & 3.473252 & 1.991601 \\ \mathrm{H} & 1.139272 & 3.378504 & 1.095388 \\ \mathrm{H} & -0.704905 & 4.306592 & -0.232029 \\ \mathrm{H} & -1.656305 & 2.850292 & -0.025982 \\ \mathrm{H} & -0.417936 & 3.214064 & -2.265327 \\ \mathrm{H} & 1.084483 & 2.920959 & -1.415329 \\ \mathrm{H} & 0.720930 & -3.623336 & -1.306411 \\ \mathrm{H} & -0.948595 & -3.650724 & -1.834436 \\ \mathrm{H} & -0.135437 & -3.354472 & 2.356377 \\ \mathrm{H} & 1.215650 & -3.223883 & 1.220723 \\ \mathrm{H} & -1.740635 & -2.988555 & 0.426991 \\ \mathrm{H} & -0.834318 & -4.482357 & 0.420988 \\ \mathrm{H} & -3.290314 & -0.216321 & 0.829390 \\ \mathrm{H} & -2.229951 & 2.140443 & -3.241384 \\ \mathrm{H} & 2.496758 & 2.425124 & 3.002214 \\ \mathrm{H} & 3.338705 & -0.412236 & -0.801949 \\ \mathrm{H} & 4.696082 & -2.887107 & -1.253168 \\ \mathrm{H} & 5.444321 & -1.279474 & -1.038770 \\ \mathrm{H} & 4.972060 & -1.853541 & -2.662070 \\ \mathrm{H} & 4.557405 & 3.418249 & 1.207706 \\ \mathrm{H} & 5.215282 & 2.213669 & 2.328730 \\ \mathrm{H} & 4.444957 & 3.691841 & 2.965711 \\ \mathrm{H} & -4.608339 & -2.594524 & 1.555132 \\ \mathrm{H} & -5.398101 & -1.021040 & 1.249578 \\ \mathrm{H} & -4.888436 & -1.477919 & 2.898604 \\ \mathrm{H} & -4.333575 & 3.293774 & -1.580900 \\ \mathrm{H} & -4.974542 & 2.123884 & -2.748054 \\ \mathrm{H} & -4.076007 & 3.554675 & -3.325443 \\ & & & \end{array}$

\section{$\left(R_{\mathrm{p}}\right)$-[3.3]pCp-5, 8, 14, 17-tetracarboxamide (Syn_chair)}

Dihedral $=-140.7^{\circ},-140.7^{\circ}, 53.3^{\circ}, 53.3^{\circ}$

Total Energy: -1529.44830413 a.u.
C $\quad$ 1. 227111
0.615143
- 1. 796797
C $\quad 0.087378 \quad 1.416999-1.732723$ 


\begin{tabular}{|c|c|c|}
\hline 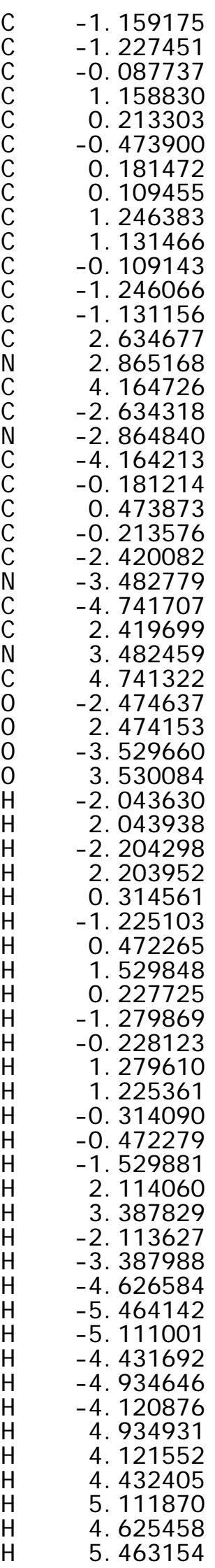 & 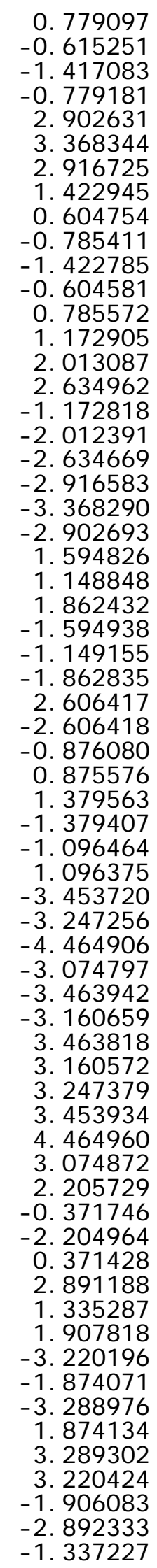 & 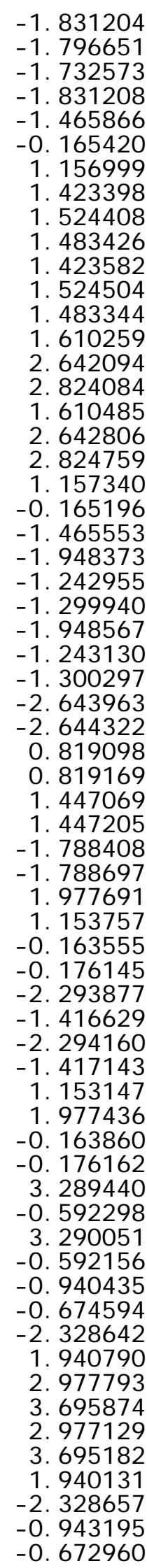 \\
\hline
\end{tabular}


$\left(\boldsymbol{R}_{\mathrm{p}}\right)-[3.3] \mathrm{pCp}-5, \mathbf{5}, \mathbf{1 4}, \mathbf{1 7}$-tetracarboxamide (Syn_boat) (have a imaginary frequency) Dihedral $=-137.2^{\circ},-141.8^{\circ}, 46.7^{\circ}, 49.2^{\circ}$

Total Energy: -1529.44785218 a.u.

$\begin{array}{rrrr}\mathrm{C} & -1.204769 & 0.717544 & 1.466966 \\ \mathrm{C} & 0.068314 & 1.282039 & 1.469443 \\ \mathrm{C} & 1.151986 & 0.390881 & 1.593763 \\ \mathrm{C} & 0.951516 & -0.984592 & 1.505330 \\ \mathrm{C} & -0.323216 & -1.544408 & 1.372416 \\ \mathrm{C} & -1.410412 & -0.665204 & 1.459984 \\ \mathrm{C} & 0.227989 & 2.776665 & 1.234864 \\ \mathrm{C} & 1.176267 & 3.178745 & 0.081857 \\ \mathrm{C} & 0.611830 & 3.007656 & -1.343054 \\ \mathrm{C} & 0.291983 & 1.565348 & -1.674614 \\ \mathrm{C} & 1.345110 & 0.648612 & -1.760469 \\ \mathrm{C} & 1.135512 & -0.726616 & -1.830987 \\ \mathrm{C} & -0.172971 & -1.237421 & -1.769391 \\ \mathrm{C} & -1.219841 & -0.320784 & -1.803354 \\ \mathrm{C} & -1.012754 & 1.064806 & -1.777493 \\ \mathrm{C} & -2.203384 & 1.989134 & -1.836479 \\ \mathrm{O} & -2.186802 & 3.042859 & -2.468719 \\ \mathrm{C} & -0.457594 & -2.717389 & -1.603606 \\ \mathrm{C} & 0.181701 & -3.363986 & -0.356526 \\ \mathrm{C} & -0.457878 & -3.009243 & 1.002615 \\ \mathrm{C} & 2.307486 & -1.661251 & -1.972336 \\ \mathrm{O} & 2.284484 & -2.618615 & -2.741811 \\ \mathrm{C} & 2.570976 & 0.854645 & 1.754681 \\ \mathrm{O} & 3.496250 & 0.424135 & 1.064946 \\ \mathrm{C} & -2.838810 & -1.128312 & 1.434021 \\ \mathrm{O} & -3.662637 & -0.682889 & 0.633959 \\ \mathrm{~N} & 2.790644 & 1.766390 & 2.725740 \\ \mathrm{C} & 4.118747 & 2.305435 & 2.956904 \\ \mathrm{~N} & -3.190996 & -2.036108 & 2.369234 \\ \mathrm{C} & -4.545994 & -2.554534 & 2.434528 \\ \mathrm{~N} & -3.302926 & 1.578804 & -1.163671 \\ \mathrm{C} & -4.518689 & 2.364335 & -1.213293 \\ \mathrm{~N} & 3.378567 & -1.381221 & -1.197337 \\ \mathrm{C} & 4.551586 & -2.229238 & -1.249259 \\ \mathrm{H} & -2.074640 & 1.368674 & 1.404330 \\ \mathrm{H} & 1.827942 & -1.631242 & 1.484854 \\ \mathrm{H} & -2.239613 & -0.701253 & -1.821285 \\ \mathrm{H} & 2.367871 & 1.024728 & -1.746291 \\ \mathrm{H} & 0.020930 & -3.628010 & 1.773785 \\ \mathrm{H} & -1.513077 & -3.299167 & 0.975881 \\ \mathrm{H} & 0.109258 & -4.452037 & -0.471559 \\ \mathrm{H} & 1.254101 & -3.140213 & -0.327372 \\ \mathrm{H} & -0.088323 & -3.256430 & -2.481072 \\ \mathrm{H} & -1.545814 & -2.859590 & -1.560045 \\ \mathrm{H} & -0.274227 & 3.631828 & -1.468780 \\ \mathrm{H} & 1.362984 & 3.383722 & -2.048874 \\ \mathrm{H} & 0.573382 & 3.275013 & 2.149572 \\ \mathrm{H} & -0.767517 & 3.190794 & 1.028858 \\ \mathrm{H} & 2.127939 & 2.639725 & 0.165699 \\ \mathrm{H} & 1.418414 & 4.240425 & 0.214314 \\ \mathrm{H} & 2.028587 & 2.020852 & 3.337271 \\ \mathrm{H} & 3.321282 & -0.665004 & -0.474926 \\ \mathrm{H} & -2.498204 & -2.338280 & 3.039410 \\ \mathrm{H} & -3.295783 & 0.727665 & -0.605847 \\ \mathrm{H} & -4.359496 & 3.361259 & -0.789003 \\ \mathrm{H} & -5.287600 & 1.842219 & -0.641355 \\ \mathrm{H} & -4.853365 & 2.491374 & -2.247428\end{array}$




$\begin{array}{rrrr}\mathrm{H} & -4.807113 & -3.055339 & 1.497921 \\ \mathrm{H} & -5.258649 & -1.741658 & 2.598594 \\ \mathrm{H} & -4.609605 & -3.266780 & 3.257603 \\ \mathrm{H} & 4.805808 & 1.522271 & 3.290781 \\ \mathrm{H} & 4.053432 & 3.083179 & 3.718458 \\ \mathrm{H} & 4.510574 & 2.734110 & 2.030670 \\ \mathrm{H} & 4.980266 & -2.230453 & -2.255749 \\ \mathrm{H} & 4.299012 & -3.264180 & -0.992505 \\ \mathrm{H} & 5.285702 & -1.844369 & -0.539733\end{array}$

$\left(R_{p}\right)$-[3.3]pCp-5, 8, 14, 17-tetracarboxamide dimer (Anti_chair) Dihedral $=-138.2^{\circ}, 44.9^{\circ}, 44.6^{\circ},-132.0^{\circ} ;-137.1^{\circ}, 40.5^{\circ}, 52.6^{\circ},-131.6^{\circ}$ Total Energy: -3058.94753552 a.u.

$\begin{array}{lrrr}\mathrm{C} & 5.246823 & -1.390060 & 0.177773 \\ \mathrm{C} & 5.156654 & -0.843982 & -1.114166 \\ \mathrm{C} & 5.168864 & 0.543212 & -1.220971 \\ \mathrm{C} & 5.134581 & 1.375714 & -0.099372 \\ \mathrm{C} & 4.998349 & 0.825636 & 1.184889 \\ \mathrm{C} & 5.125717 & -0.562024 & 1.292917 \\ \mathrm{C} & 4.942377 & -1.707251 & -2.338635 \\ \mathrm{C} & 3.682172 & -2.595641 & -2.260065 \\ \mathrm{C} & 2.319455 & -1.880780 & -2.370382 \\ \mathrm{C} & 1.998160 & -0.943761 & -1.219725 \\ \mathrm{C} & 1.869788 & 0.449751 & -1.364026 \\ \mathrm{C} & 1.807861 & 1.269114 & -0.236936 \\ \mathrm{C} & 1.830875 & 0.759305 & 1.064621 \\ \mathrm{C} & 1.810089 & -0.636333 & 1.203370 \\ \mathrm{C} & 1.913155 & -1.454499 & 0.073738 \\ \mathrm{C} & 1.789720 & -1.343854 & 2.532149 \\ \mathrm{O} & 2.581669 & -2.265905 & 2.777197 \\ \mathrm{C} & 1.985847 & 1.732739 & 2.218488 \\ \mathrm{C} & 3.323488 & 2.500428 & 2.214393 \\ \mathrm{C} & 4.593087 & 1.640932 & 2.402866 \\ \mathrm{C} & 1.786249 & 1.082054 & -2.723898 \\ \mathrm{~N} & 2.546412 & 2.162314 & -2.935618 \\ \mathrm{C} & 2.487540 & 2.874877 & -4.197338 \\ \mathrm{C} & 5.160543 & 2.849313 & -0.360153 \\ \mathrm{~N} & 6.006846 & 3.578648 & 0.396099 \\ \mathrm{C} & 6.118390 & 5.015016 & 0.218694 \\ \mathrm{C} & 5.449145 & -2.873784 & 0.356301 \\ \mathrm{~N} & 4.713965 & -3.458118 & 1.322227 \\ \mathrm{C} & 4.837350 & -4.881607 & 1.560699 \\ \mathrm{~N} & 0.866712 & -0.958972 & 3.419749 \\ \mathrm{C} & 0.781482 & -1.587214 & 4.723592 \\ \mathrm{O} & 6.247313 & -3.493839 & -0.343140 \\ \mathrm{O} & 4.465375 & 3.387765 & -1.228476 \\ \mathrm{O} & 1.023420 & 0.638123 & -3.597969 \\ \mathrm{O} & -1.077555 & 1.082103 & 3.514167 \\ \mathrm{C} & -1.767872 & 1.437308 & 2.546105 \\ \mathrm{~N} & -2.447610 & 2.592173 & 2.549916 \\ \mathrm{C} & -2.401049 & 3.470122 & 3.702580 \\ \mathrm{C} & -1.860994 & 0.601595 & 1.304716 \\ \mathrm{C} & -2.038767 & -0.791569 & 1.386627 \\ \mathrm{C} & -1.931695 & -1.507117 & 0.196729 \\ \mathrm{C} & -1.794617 & -0.883272 & -1.047209 \\ \mathrm{C} & -1.839300 & 0.516371 & -1.140689 \\ \mathrm{C} & -1.798162 & 1.227136 & 0.060725 \\ \mathrm{C} & -2.467713 & -1.494272 & 2.661439 \\ \mathrm{C} & -3.765984 & -0.949130 & 3.295202 \\ \mathrm{C} & -5.079386 & -1.280803 & 2.560131 \\ \mathrm{C} & -5.199195 & -0.638405 & 1.193347\end{array}$




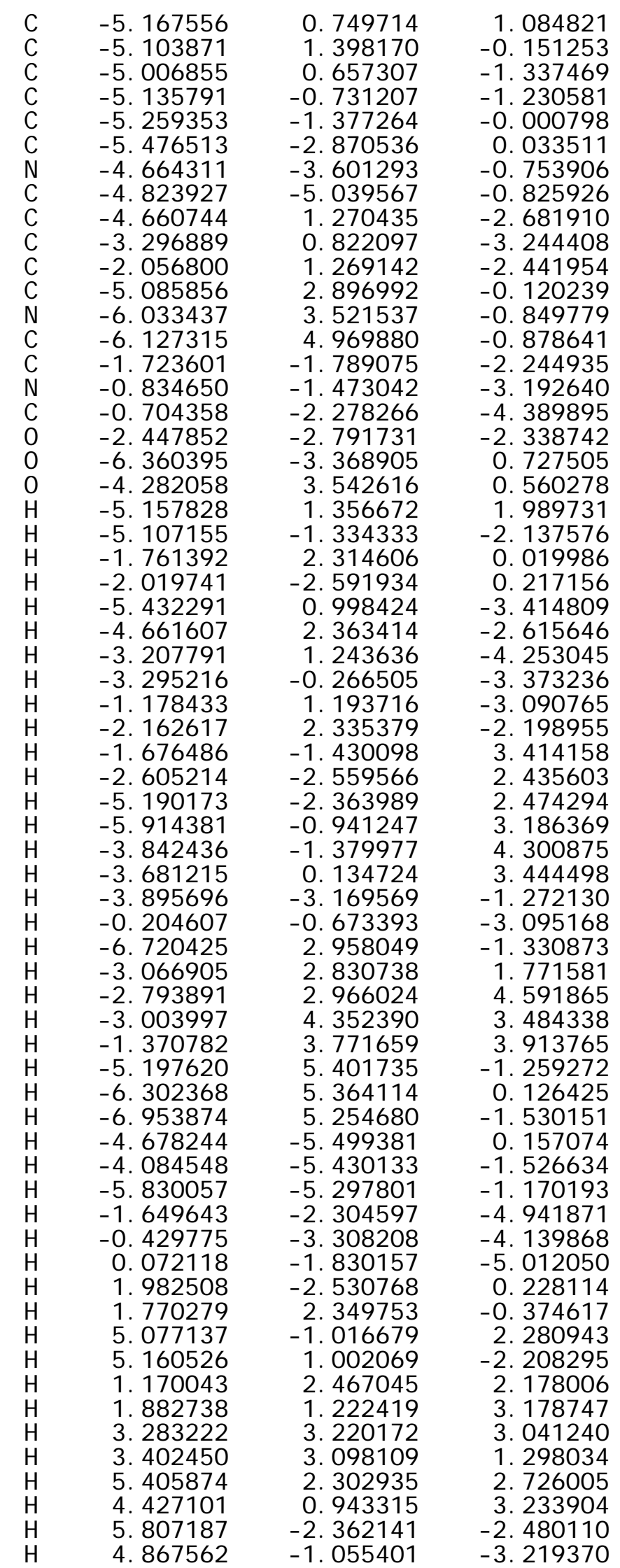




$\begin{array}{rrrr}\mathrm{H} & 2.272727 & -1.340927 & -3.320785 \\ \mathrm{H} & 1.541434 & -2.654304 & -2.418552 \\ \mathrm{H} & 3.735614 & -3.313629 & -3.087173 \\ \mathrm{H} & 3.711638 & -3.197830 & -1.344124 \\ \mathrm{H} & 3.201956 & 2.489400 & -2.221289 \\ \mathrm{H} & 6.655547 & 3.092505 & 0.998740 \\ \mathrm{H} & 0.198672 & -0.211161 & 3.211058 \\ \mathrm{H} & 3.976804 & -2.938103 & 1.803401 \\ \mathrm{H} & 4.568651 & -5.454151 & 0.665898 \\ \mathrm{H} & 4.171297 & -5.151209 & 2.381561 \\ \mathrm{H} & 5.867783 & -5.137231 & 1.824052 \\ \mathrm{H} & 1.737491 & -1.512232 & 5.250588 \\ \mathrm{H} & 0.528192 & -2.648228 & 4.628681 \\ \mathrm{H} & 0.006459 & -1.072282 & 5.294030 \\ \mathrm{H} & 2.732814 & 2.208117 & -5.029292 \\ \mathrm{H} & 3.206705 & 3.694036 & -4.158257 \\ \mathrm{H} & 1.483862 & 3.276178 & -4.368494 \\ \mathrm{H} & 5.136320 & 5.479966 & 0.338420 \\ \mathrm{H} & 6.493921 & 5.258136 & -0.779953 \\ \mathrm{H} & 6.801345 & 5.409998 & 0.971441\end{array}$

\section{$\left(R_{p}\right)$-[3.3]pCp-5, 8, 14, 17-tetracarboxamide dimer (Anti_boat)}

Dihedral $=-134.4^{\circ}, 49.4^{\circ}, 45.0^{\circ},-138.1^{\circ} ;-133.0^{\circ}, 42.2^{\circ}, 47.7^{\circ},-137.5^{\circ}$

Total Energy: -3058.94883700 a.u.

$\begin{array}{lrrr}\mathrm{C} & 5.062500 & 0.710292 & -1.210235 \\ \mathrm{C} & 5.062611 & -0.678725 & -1.231730 \\ \mathrm{C} & 5.228590 & -1.341485 & -0.000001 \\ \mathrm{C} & 5.210040 & -0.620400 & 1.189591 \\ \mathrm{C} & 5.094000 & 0.775860 & 1.216739 \\ \mathrm{C} & 5.112436 & 1.438708 & -0.015321 \\ \mathrm{C} & 4.818402 & -1.425750 & -2.526850 \\ \mathrm{C} & 3.594522 & -2.366646 & -2.508930 \\ \mathrm{C} & 2.208510 & -1.690935 & -2.551245 \\ \mathrm{C} & 1.907249 & -0.853196 & -1.323573 \\ \mathrm{C} & 1.803250 & 0.546648 & -1.334351 \\ \mathrm{C} & 1.836817 & 1.259082 & -0.131491 \\ \mathrm{C} & 1.925683 & 0.634376 & 1.110970 \\ \mathrm{C} & 1.841780 & -0.769075 & 1.121239 \\ \mathrm{C} & 1.861001 & -1.480840 & -0.077177 \\ \mathrm{C} & 1.833738 & -1.577738 & 2.389136 \\ \mathrm{O} & 2.589461 & -2.548412 & 2.544528 \\ \mathrm{C} & 2.201641 & 1.470379 & 2.349562 \\ \mathrm{C} & 3.454591 & 1.060834 & 3.153922 \\ \mathrm{C} & 4.810546 & 1.463423 & 2.538117 \\ \mathrm{C} & 1.680695 & 1.315243 & -2.618487 \\ \mathrm{~N} & 2.384021 & 2.453093 & -2.708433 \\ \mathrm{C} & 2.283047 & 3.291764 & -3.886805 \\ \mathrm{C} & 5.439038 & -2.834839 & 0.024597 \\ \mathrm{O} & 6.223076 & -3.375413 & -0.752558 \\ \mathrm{C} & 5.101518 & 2.931388 & -0.145536 \\ \mathrm{O} & 4.278741 & 3.534644 & -0.842384 \\ \mathrm{~N} & 0.968250 & -1.199364 & 3.335048 \\ \mathrm{C} & 0.899527 & -1.897706 & 4.603195 \\ \mathrm{~N} & 4.727314 & -3.515608 & 0.942957 \\ \mathrm{C} & 4.851732 & -4.956400 & 1.031089 \\ \mathrm{~N} & 6.074930 & 3.597605 & 0.510138 \\ \mathrm{C} & 6.181714 & 5.043148 & 0.430122 \\ \mathrm{O} & 0.938698 & 0.939203 & -3.539976 \\ \mathrm{O} & -0.985936 & 0.823757 & 3.471671 \\ \mathrm{C} & -1.729197 & 1.242243 & 2.569094 \\ \mathrm{~N} & -2.458741 & 2.354363 & 2.715334\end{array}$




\begin{tabular}{|c|c|c|}
\hline 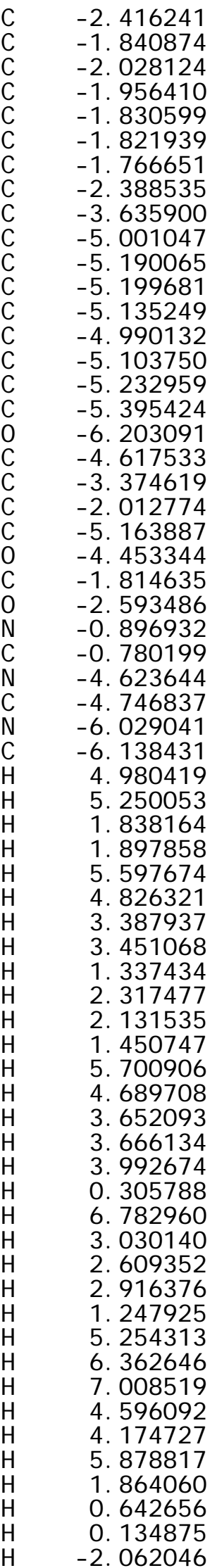 & 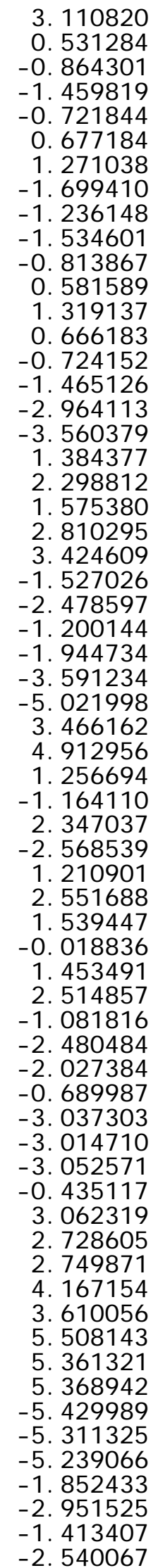 & 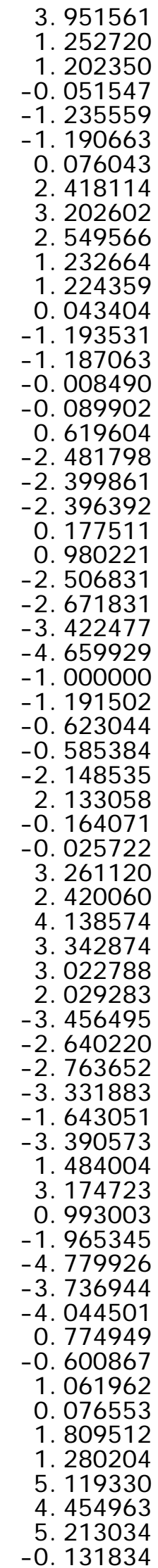 \\
\hline
\end{tabular}




$\begin{array}{rrrr}\mathrm{H} & -1.696268 & 2.356875 & 0.141134 \\ \mathrm{H} & -5.037407 & -1.255884 & -2.134518 \\ \mathrm{H} & -5.218171 & 1.121289 & 2.170873 \\ \mathrm{H} & -1.218215 & 2.332657 & -2.415480 \\ \mathrm{H} & -1.913324 & 1.011998 & -3.327054 \\ \mathrm{H} & -3.388596 & 2.957811 & -3.276678 \\ \mathrm{H} & -3.440290 & 2.958527 & -1.525878 \\ \mathrm{H} & -5.457941 & 1.987142 & -2.848389 \\ \mathrm{H} & -4.441904 & 0.621724 & -3.251612 \\ \mathrm{H} & -5.120033 & -2.610729 & 2.410401 \\ \mathrm{H} & -5.788621 & -1.224117 & 3.248035 \\ \mathrm{H} & -1.543457 & -1.720549 & 3.113423 \\ \mathrm{H} & -2.547807 & -2.732848 & 2.083671 \\ \mathrm{H} & -3.557277 & -0.165673 & 3.430155 \\ \mathrm{H} & -3.620351 & -1.747579 & 4.172604 \\ \mathrm{H} & -3.130629 & 2.632534 & 1.995111 \\ \mathrm{H} & -6.682746 & 2.925368 & -1.171270 \\ \mathrm{H} & -0.243839 & -0.425826 & -3.265464 \\ \mathrm{H} & -3.921256 & -3.083622 & -1.542091 \\ \mathrm{H} & -4.506847 & -5.562497 & -0.269963 \\ \mathrm{H} & -4.056746 & -5.321578 & -1.981750 \\ \mathrm{H} & -5.769863 & -5.285340 & -1.477103 \\ \mathrm{H} & -1.719781 & -1.916756 & -5.220204 \\ \mathrm{H} & -0.536170 & -2.993450 & -4.460470 \\ \mathrm{H} & 0.014684 & -1.489377 & -5.252897 \\ \mathrm{H} & -2.704535 & 2.482090 & 4.800233 \\ \mathrm{H} & -3.110353 & 3.947361 & 3.861991 \\ \mathrm{H} & -1.406955 & 3.489912 & 4.137746 \\ \mathrm{H} & -5.150527 & 5.359756 & -0.723175 \\ \mathrm{H} & -6.538930 & 5.253903 & 0.374245 \\ \mathrm{H} & -6.798187 & 5.236197 & -1.391361\end{array}$

$\left(R_{\mathrm{p}}\right)$-[3.3]pCp-5, 8, 14, 17-tetracarboxamide dimer (Anti_chair_boat) Dihedral $=-131.7^{\circ}, 53.5^{\circ}, 39.9^{\circ},-138.0^{\circ} ;-132.3^{\circ}, 38.6^{\circ}, 47.9^{\circ},-136.8^{\circ}$

Total Energy: -3058.94652133 a.u.

$\begin{array}{lrrr}\mathrm{C} & 5.259977 & -1.331665 & 0.104663 \\ \mathrm{C} & 5.230938 & -0.613528 & -1.102827 \\ \mathrm{C} & 5.156334 & 0.775002 & -1.019609 \\ \mathrm{C} & 5.020694 & 1.440313 & 0.201615 \\ \mathrm{C} & 4.893475 & 0.715566 & 1.394931 \\ \mathrm{C} & 5.065619 & -0.670249 & 1.317216 \\ \mathrm{C} & 5.183106 & -1.281143 & -2.461640 \\ \mathrm{C} & 3.880353 & -1.013242 & -3.239801 \\ \mathrm{C} & 2.585987 & -1.595270 & -2.631161 \\ \mathrm{C} & 2.083613 & -0.878354 & -1.390848 \\ \mathrm{C} & 1.871094 & 0.511856 & -1.342537 \\ \mathrm{C} & 1.733765 & 1.156068 & -0.114412 \\ \mathrm{C} & 1.735316 & 0.466702 & 1.099993 \\ \mathrm{C} & 1.729573 & -0.935153 & 1.031443 \\ \mathrm{C} & 1.936921 & -1.575699 & -0.194452 \\ \mathrm{C} & 1.812240 & 1.318497 & -2.604973 \\ \mathrm{~N} & 2.438541 & 2.503232 & -2.600288 \\ \mathrm{C} & 2.414111 & 3.351270 & -3.776029 \\ \mathrm{C} & 1.658326 & -1.821975 & 2.244253 \\ \mathrm{O} & 2.417281 & -2.794666 & 2.370877 \\ \mathrm{C} & 1.882110 & 1.247351 & 2.394953 \\ \mathrm{C} & 3.112713 & 0.857521 & 3.242008 \\ \mathrm{C} & 4.477783 & 1.342065 & 2.713347 \\ \mathrm{C} & 4.961130 & 2.937619 & 0.148757 \\ \mathrm{O} & 4.158779 & 3.551292 & -0.562400 \\ \mathrm{C} & 5.521663 & -2.818501 & 0.104888\end{array}$




\begin{tabular}{|c|c|c|}
\hline 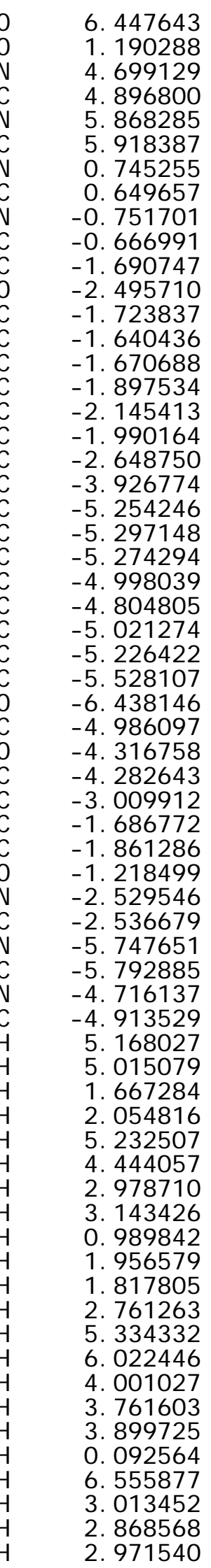 & 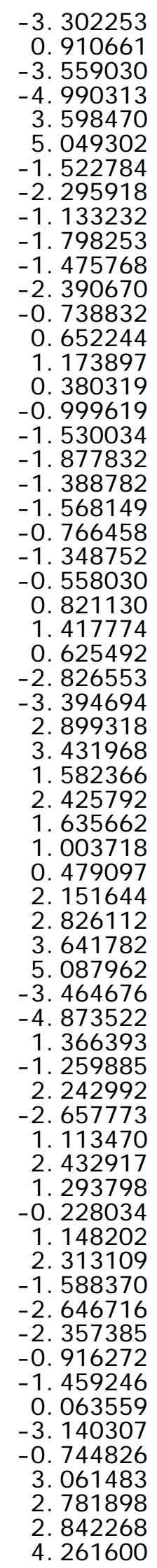 & 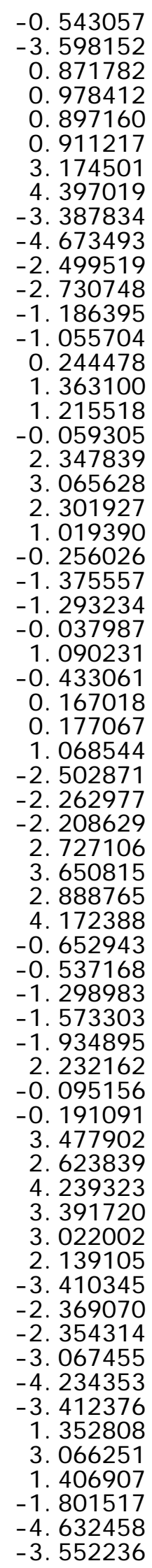 \\
\hline
\end{tabular}




$\begin{array}{rrrr}\mathrm{H} & 1.384325 & 3.606682 & -4.043013 \\ \mathrm{H} & 4.969391 & 5.458205 & 1.269086 \\ \mathrm{H} & 6.101302 & 5.436941 & -0.094940 \\ \mathrm{H} & 6.723322 & 5.365772 & 1.575106 \\ \mathrm{H} & 4.798501 & -5.472819 & 0.000415 \\ \mathrm{H} & 4.144499 & -5.388315 & 1.660990 \\ \mathrm{H} & 5.897081 & -5.212060 & 1.362813 \\ \mathrm{H} & 1.609816 & -2.300738 & 4.923034 \\ \mathrm{H} & 0.374955 & -3.333757 & 4.183040 \\ \mathrm{H} & -0.112145 & -1.832879 & 5.026592 \\ \mathrm{H} & -2.149177 & -2.595537 & -0.212397 \\ \mathrm{H} & -1.547556 & 2.248989 & 0.378337 \\ \mathrm{H} & -4.881857 & -1.042746 & -2.343151 \\ \mathrm{H} & -5.294295 & 1.115291 & 2.061349 \\ \mathrm{H} & -0.861177 & 2.353902 & -2.106850 \\ \mathrm{H} & -1.540402 & 1.138492 & -3.169010 \\ \mathrm{H} & -2.925009 & 3.144314 & -3.087476 \\ \mathrm{H} & -3.114005 & 3.023858 & -1.350075 \\ \mathrm{H} & -5.056468 & 2.246627 & -2.907430 \\ \mathrm{H} & -4.076896 & 0.850791 & -3.295242 \\ \mathrm{H} & -5.426625 & -2.625332 & 2.091523 \\ \mathrm{H} & -6.072253 & -1.246494 & 2.959065 \\ \mathrm{H} & -1.864400 & -1.985466 & 3.103918 \\ \mathrm{H} & -2.833522 & -2.880894 & 1.941977 \\ \mathrm{H} & -3.811081 & -0.339061 & 3.361947 \\ \mathrm{H} & -4.009970 & -1.952810 & 4.002406 \\ \mathrm{H} & -3.125296 & 2.515070 & 2.139901 \\ \mathrm{H} & -6.380054 & 3.166757 & -1.281324 \\ \mathrm{H} & -0.072990 & -0.391650 & -3.199259 \\ \mathrm{H} & -3.938395 & -2.980451 & -1.751297 \\ \mathrm{H} & -4.836608 & -5.462744 & -0.653893 \\ \mathrm{H} & -4.147314 & -5.195200 & -2.280263 \\ \mathrm{H} & -5.905217 & -5.048945 & -2.001995 \\ \mathrm{H} & -1.622905 & -1.736688 & -5.202026 \\ \mathrm{H} & -0.415083 & -2.856585 & -4.549060 \\ \mathrm{H} & 0.108749 & -1.299901 & -5.257575 \\ \mathrm{H} & -2.964658 & 2.181871 & 4.947621 \\ \mathrm{H} & -3.134821 & 3.733306 & 4.077923 \\ \mathrm{H} & -1.518032 & 3.087232 & 4.473509 \\ \mathrm{H} & -4.781433 & 5.496823 & -0.607681 \\ \mathrm{H} & -6.220602 & 5.392048 & 0.423000 \\ \mathrm{H} & -6.401753 & 5.485562 & -1.349887 \\ & & & \end{array}$

\section{$\left(R_{p}\right)$-[3.3]pCp-5, 8, 14, 17-tetracarboxamide dimer (Syn_chair)}

Dihedral $=-137.4^{\mathrm{o}},-138.9^{\circ}, 38.2^{\circ}, 43.9^{\circ} ;-137.2^{\circ},-141.8^{\mathrm{o}}, \overline{51} .3^{\circ}, 54.7^{\circ}$

Total Energy: -3058.93646342 a.u.

$\begin{array}{lrrr}\mathrm{C} & 1.599023 & -0.666330 & 1.082865 \\ \mathrm{C} & 1.663877 & 0.733491 & 0.995289 \\ \mathrm{C} & 1.717081 & 1.290652 & -0.282778 \\ \mathrm{C} & 1.845179 & 0.511299 & -1.432317 \\ \mathrm{C} & 1.991267 & -0.881717 & -1.322801 \\ \mathrm{C} & 1.792537 & -1.443810 & -0.063075 \\ \mathrm{C} & 1.787236 & 1.634841 & 2.207122 \\ \mathrm{C} & 2.984041 & 1.312590 & 3.125349 \\ \mathrm{C} & 4.376313 & 1.688715 & 2.578056 \\ \mathrm{C} & 4.805771 & 0.886130 & 1.362853 \\ \mathrm{C} & 4.911855 & -0.502658 & 1.458549 \\ \mathrm{C} & 5.110833 & -1.309443 & 0.337883 \\ \mathrm{C} & 5.137971 & -0.752216 & -0.950460 \\ \mathrm{C} & 5.135925 & 0.640417 & -1.034905 \\ \mathrm{C} & 5.009703 & 1.452073 & 0.093559\end{array}$




\begin{tabular}{|c|c|c|c|}
\hline $\begin{array}{l}\mathrm{N} \\
\mathrm{C} \\
\mathrm{C} \\
\mathrm{N} \\
\mathrm{C} \\
\mathrm{O} \\
\mathrm{O} \\
\mathrm{O} \\
\mathrm{O} \\
\mathrm{N} \\
\mathrm{C} \\
\mathrm{C} \\
\mathrm{O} \\
\mathrm{C} \\
\mathrm{C}\end{array}$ & 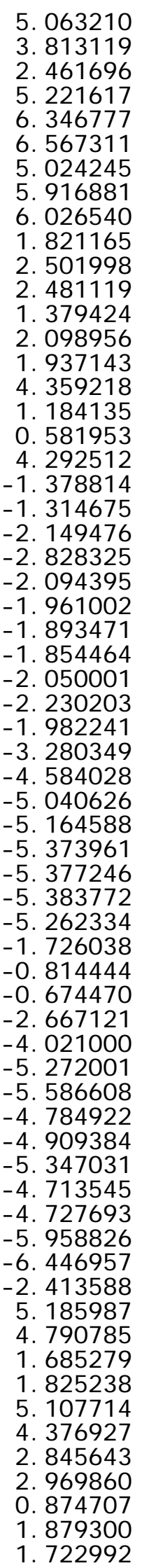 & 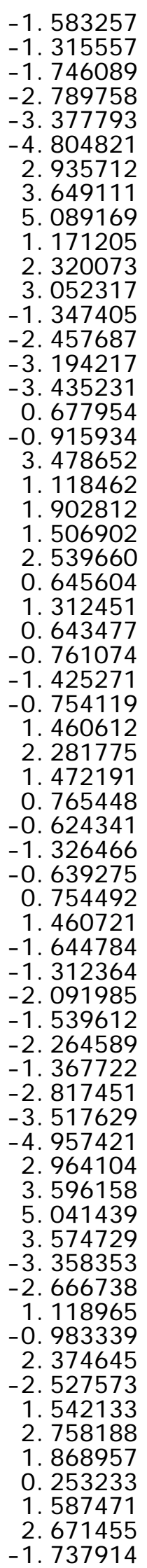 & 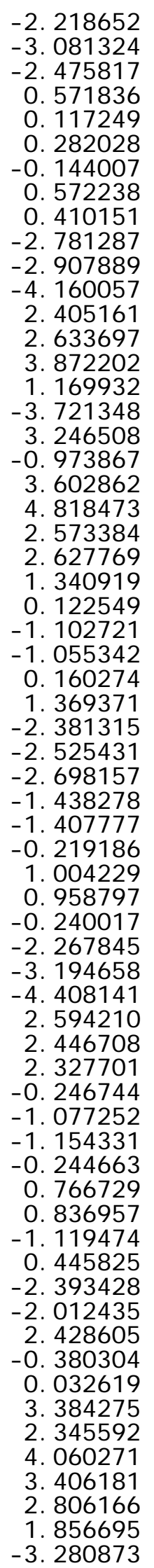 \\
\hline
\end{tabular}




$\begin{array}{rrrr}\mathrm{H} & 2.540143 & -2.782976 & -2.123522 \\ \mathrm{H} & 5.089438 & -2.650132 & -1.974173 \\ \mathrm{H} & 5.948921 & -1.380821 & -2.835944 \\ \mathrm{H} & 3.946996 & -1.866939 & -4.019492 \\ \mathrm{H} & 3.776622 & -0.256271 & -3.362352 \\ \mathrm{H} & 7.049403 & -2.804256 & -0.327911 \\ \mathrm{H} & 2.834497 & -2.744048 & 1.987052 \\ \mathrm{H} & 6.542965 & 3.160062 & 1.196491 \\ \mathrm{H} & 3.075742 & 2.667310 & -2.138320 \\ \mathrm{H} & 2.912069 & 2.453423 & -4.969044 \\ \mathrm{H} & 3.061284 & 3.967023 & -4.032067 \\ \mathrm{H} & 1.453585 & 3.304571 & -4.436872 \\ \mathrm{H} & 5.060078 & 5.562928 & 0.600658 \\ \mathrm{H} & 6.336564 & 5.339249 & -0.608374 \\ \mathrm{H} & 6.763506 & 5.466870 & 1.119101 \\ \mathrm{H} & 6.579970 & -5.070002 & 1.342588 \\ \mathrm{H} & 7.524538 & -5.065128 & -0.170188 \\ \mathrm{H} & 5.767688 & -5.370031 & -0.204379 \\ \mathrm{H} & 0.901082 & -3.525168 & 3.987014 \\ \mathrm{H} & 2.189638 & -2.568530 & 4.734778 \\ \mathrm{H} & 2.597129 & -4.062059 & 3.842878 \\ \mathrm{H} & -1.961460 & 2.402255 & 0.134373 \\ \mathrm{H} & -2.121697 & -2.511970 & 0.137579 \\ \mathrm{H} & -5.454534 & 1.309400 & 1.893270 \\ \mathrm{H} & -5.066962 & -1.181728 & -2.338578 \\ \mathrm{H} & -1.897324 & -2.288220 & 2.824570 \\ \mathrm{H} & -2.723580 & -0.883930 & 3.466886 \\ \mathrm{H} & -4.146335 & -2.891655 & 3.338213 \\ \mathrm{H} & -3.980930 & -2.958381 & 1.599170 \\ \mathrm{H} & -6.153420 & -1.999949 & 2.470070 \\ \mathrm{H} & -5.258396 & -0.626404 & 3.138127 \\ \mathrm{H} & -5.363509 & 2.161709 & -3.035029 \\ \mathrm{H} & -4.436230 & 0.722998 & -3.487549 \\ \mathrm{H} & -1.889213 & 0.820110 & -3.261122 \\ \mathrm{H} & -1.131247 & 2.156203 & -2.418133 \\ \mathrm{H} & -3.162235 & 2.913609 & -3.414416 \\ \mathrm{H} & -3.380817 & 2.973133 & -1.680612 \\ \mathrm{H} & -0.217146 & -0.492781 & -3.095878 \\ \mathrm{H} & -3.984116 & -3.072665 & -1.527511 \\ \mathrm{H} & -0.753437 & 0.320640 & 3.479667 \\ \mathrm{H} & -4.099314 & 3.082403 & 1.399792 \\ \mathrm{H} & -4.296110 & 5.483453 & -0.068730 \\ \mathrm{H} & -4.145834 & 5.347636 & 1.708025 \\ \mathrm{H} & -5.752680 & 5.412615 & 0.931694 \\ \mathrm{H} & -2.311431 & 2.019005 & 5.253490 \\ \mathrm{H} & -0.916258 & 2.904390 & 4.619841 \\ \mathrm{H} & -0.665620 & 1.386103 & 5.527593 \\ \mathrm{H} & -0.296017 & -3.096784 & -4.189128 \\ \mathrm{H} & 0.023278 & -1.568459 & -5.064830 \\ \mathrm{H} & -1.641848 & -2.198626 & -4.907586 \\ \mathrm{H} & -5.911031 & -5.238631 & -1.493190 \\ \mathrm{H} & -4.748195 & -5.419806 & -0.173456 \\ \mathrm{H} & -4.165955 & -5.328712 & -1.861758 \\ & & & \end{array}$

$\left(R_{p}\right)$-[3.3]pCp-5, 8, 14, 17-tetracarboxamide dimer (Syn_boat)

Dihedral $=-135.2^{\circ},-140.7^{\circ}, 40.0^{\circ}, 42.0^{\circ} ;-133.9^{\circ},-141.4^{\circ}, 47.2^{\circ}, 49.0^{\circ}$

Total Energy: -3058.93897400 a.u.
C $\quad-5.029117$
1. 363439
0.046875
$\begin{array}{ll}C & -4.799840 \\ \text { C } & -4.889464\end{array}$
0.832898
- 1. 237802
$-0.550724$
$-1.366773$
C -5.086574
- 1.394829
- 0.270094 


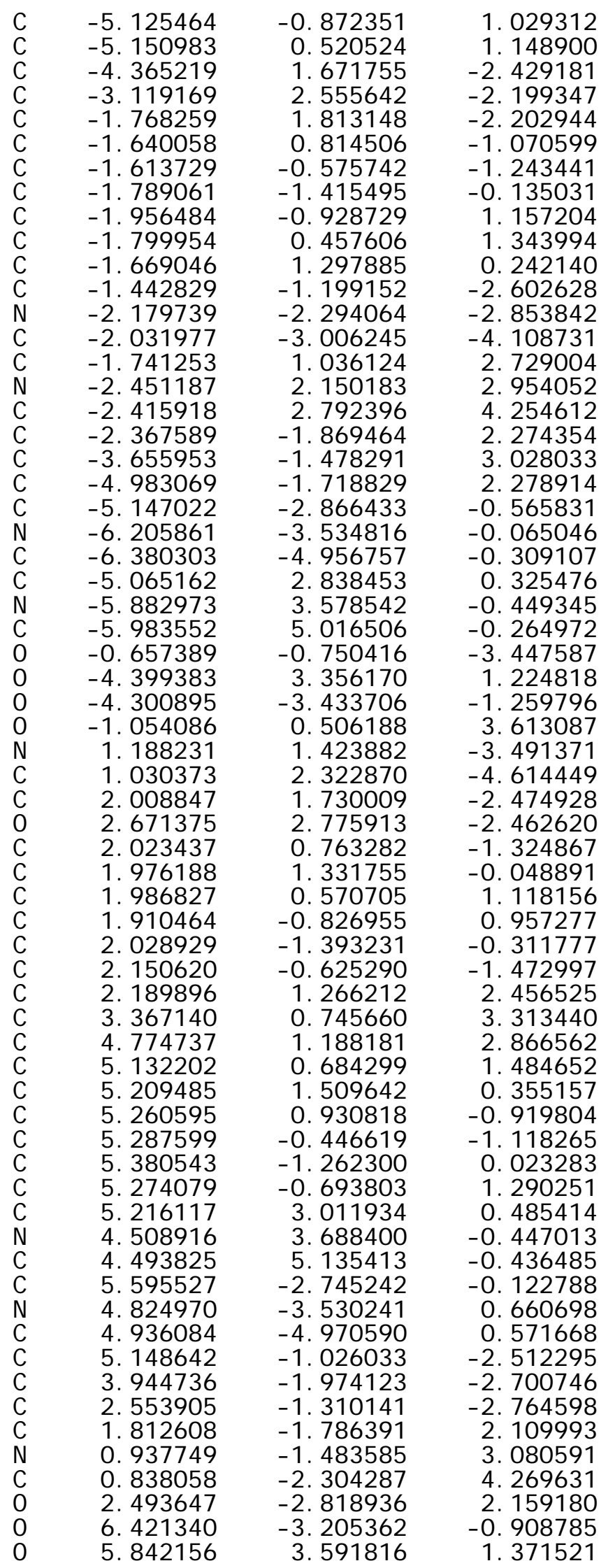




\begin{tabular}{|c|c|c|}
\hline 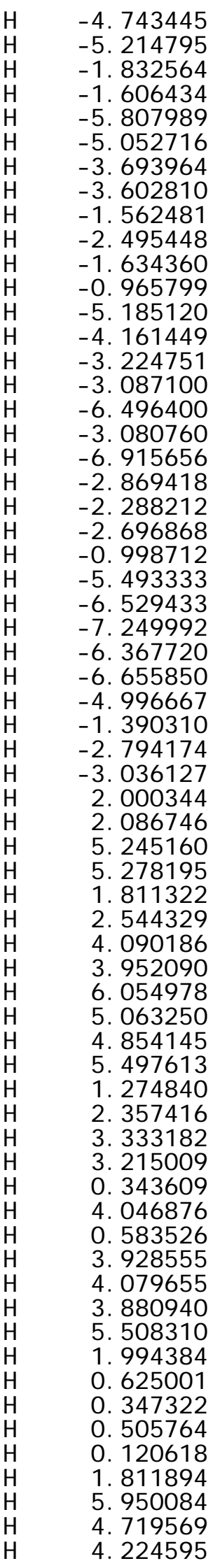 & 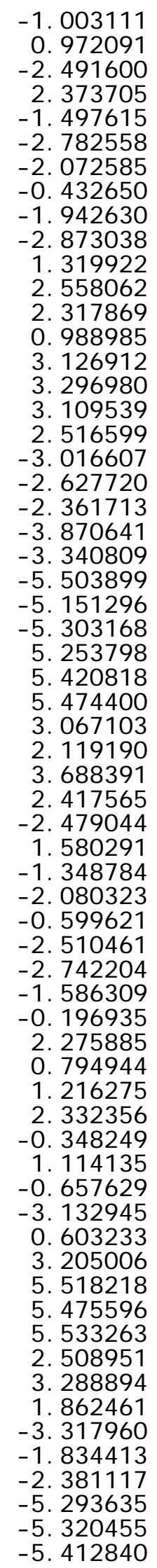 & 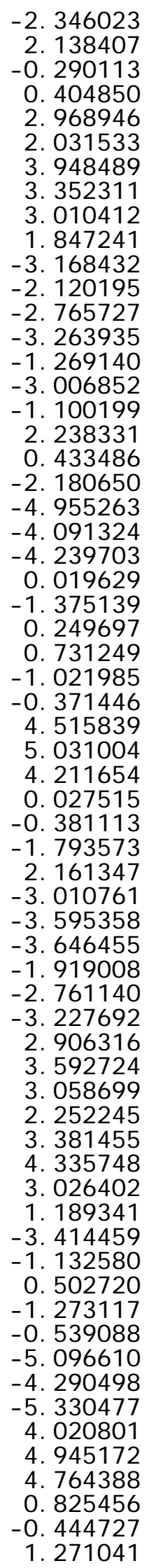 \\
\hline
\end{tabular}


$\left(R_{p}\right)$-[3.3]pCp-5, 8, 14, 17-tetracarboxamide dimer (Syn_boat_chair)

Dihedral $=-137.9^{\circ},-138.5^{\circ}, 39.3^{\circ}, 43.1^{\circ} ;-134.3^{\circ},-141.2^{\circ}, 47.5^{\circ}, 49.0^{\circ}$

Total Energy: -3058.93833198 a.u.

\begin{tabular}{lrrr}
$\mathrm{C}$ & -4.882828 & -0.511042 & -1.404619 \\
$\mathrm{C}$ & -4.765206 & 0.871089 & -1.283619 \\
$\mathrm{C}$ & -5.001182 & 1.416069 & -0.006187 \\
$\mathrm{C}$ & -5.158490 & 0.584222 & 1.099463 \\
$\mathrm{C}$ & -5.163815 & -0.809647 & 0.989460 \\
$\mathrm{C}$ & -5.115890 & -1.342591 & -0.305327 \\
$\mathrm{C}$ & -4.294235 & 1.692569 & -2.472865 \\
$\mathrm{C}$ & -3.030168 & 2.546062 & -2.228431 \\
$\mathrm{C}$ & -1.697562 & 1.772064 & -2.208946 \\
$\mathrm{C}$ & -1.605261 & 0.780048 & -1.067140 \\
$\mathrm{C}$ & -1.603633 & -0.611769 & -1.227164 \\
$\mathrm{C}$ & -1.816625 & -1.437520 & -0.114558 \\
$\mathrm{C}$ & -1.997444 & -0.935446 & 1.169960 \\
$\mathrm{C}$ & -1.813401 & 0.448828 & 1.347641 \\
$\mathrm{C}$ & -1.644701 & 1.275481 & 0.240854 \\
$\mathrm{C}$ & -1.426824 & -1.250979 & -2.578205 \\
$\mathrm{~N}$ & -2.189558 & -2.327045 & -2.832815 \\
$\mathrm{C}$ & -2.043211 & -3.053528 & -4.079713 \\
$\mathrm{C}$ & -1.758477 & 1.038080 & 2.728275 \\
$\mathrm{~N}$ & -2.430267 & 2.180802 & 2.929517 \\
$\mathrm{C}$ & -2.390926 & 2.835702 & 4.223577 \\
$\mathrm{C}$ & -2.450883 & -1.857515 & 2.286415 \\
$\mathrm{C}$ & -3.742301 & -1.431283 & 3.015751 \\
$\mathrm{C}$ & -5.061767 & -1.649661 & 2.247328 \\
$\mathrm{C}$ & -5.007522 & 2.893741 & 0.260977 \\
$\mathrm{O}$ & -4.339855 & 3.404268 & 1.162930 \\
$\mathrm{C}$ & -5.204842 & -2.814640 & -0.591125 \\
$\mathrm{O}$ & -4.361328 & -3.405874 & -1.268062 \\
$\mathrm{O}$ & -0.615554 & -0.830288 & -3.413251 \\
$\mathrm{~N}$ & -5.801504 & 3.644548 & -0.528368 \\
$\mathrm{C}$ & -5.873996 & 5.085810 & -0.357260 \\
$\mathrm{~N}$ & -6.285106 & -3.455660 & -0.100412 \\
$\mathrm{C}$ & -6.487679 & -4.875369 & -0.335559 \\
$\mathrm{O}$ & -1.106098 & 0.493839 & 3.629047 \\
$\mathrm{~N}$ & 0.840834 & -1.538653 & 3.138795 \\
$\mathrm{C}$ & 0.684727 & -2.403392 & 4.290754 \\
$\mathrm{C}$ & 1.757278 & -1.810730 & 2.196941 \\
$\mathrm{O}$ & 2.446879 & -2.837121 & 2.255026 \\
$\mathrm{C}$ & 1.876275 & -0.846905 & 1.047151 \\
$\mathrm{C}$ & 1.920226 & 0.550423 & 1.195536 \\
$\mathrm{C}$ & 1.950074 & 1.305300 & 0.020268 \\
$\mathrm{C}$ & 2.051187 & 0.726444 & -1.245336 \\
$\mathrm{C}$ & 2.193211 & -0.666116 & -1.378361 \\
$\mathrm{C}$ & 2.043444 & -1.423035 & -0.216402 \\
$\mathrm{C}$ & 2.049057 & 1.270663 & 2.527598 \\
$\mathrm{C}$ & 3.347146 & 2.088508 & 2.689176 \\
$\mathrm{C}$ & 4.659413 & 1.278071 & 2.760058 \\
$\mathrm{C}$ & 5.072400 & 0.656482 & 1.441891 \\
$\mathrm{C}$ & 5.191943 & -0.728014 & 1.315806 \\
$\mathrm{C}$ & 5.367547 & -1.350027 & 0.078040 \\
$\mathrm{C}$ & 5.345803 & -0.582878 & -1.096991 \\
$\mathrm{C}$ & 5.353846 & 0.804718 & -0.959115 \\
$\mathrm{C}$ & 5.260201 & 1.429858 & 0.286292 \\
$\mathrm{O}$ & 5.339180 & 2.930346 & 0.383323 \\
& 5.975934 & 3.489677 & 1.274414 \\
3.947799 & -1.221447 & -2.462990 \\
& -2.100927 & -2.611273 \\
\hline
\end{tabular}




\begin{tabular}{|c|c|c|}
\hline 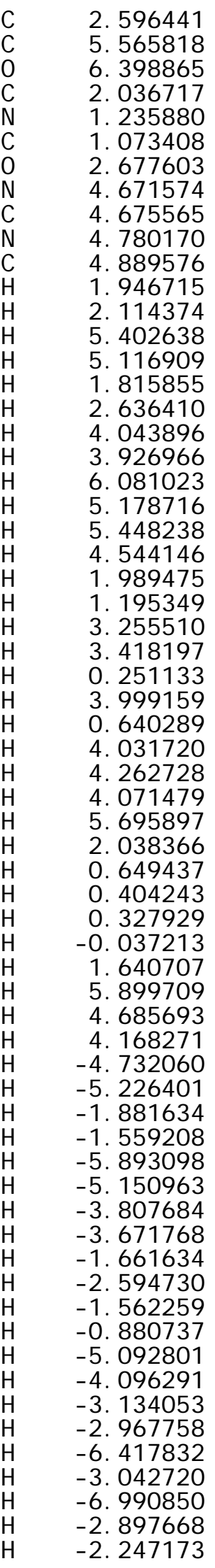 & 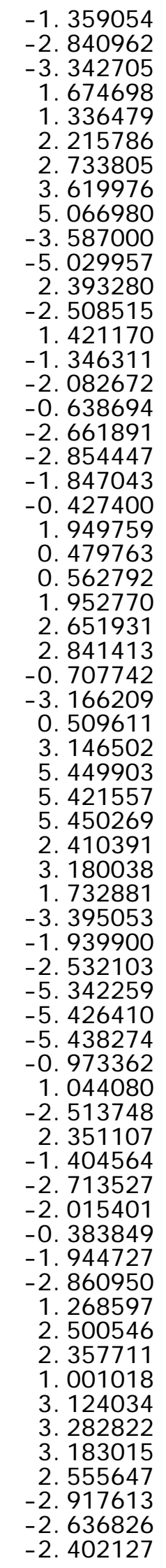 & 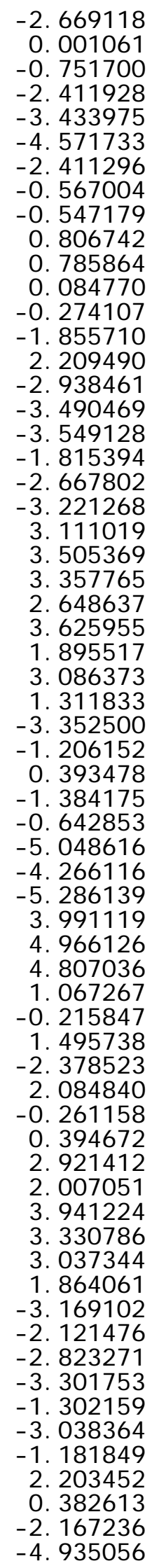 \\
\hline
\end{tabular}




$\begin{array}{rrrr}\mathrm{H} & -2.749045 & -3.885091 & -4.074854 \\ \mathrm{H} & -1.024418 & -3.438310 & -4.183053 \\ \mathrm{H} & -5.610452 & -5.436684 & -0.004894 \\ \mathrm{H} & -6.642625 & -5.074319 & -1.400006 \\ \mathrm{H} & -7.362528 & -5.201718 & 0.227247 \\ \mathrm{H} & -6.258082 & 5.339536 & 0.634906 \\ \mathrm{H} & -6.534720 & 5.496435 & -1.121077 \\ \mathrm{H} & -4.877933 & 5.523571 & -0.463187 \\ \mathrm{H} & -1.360374 & 3.081751 & 4.494269 \\ \mathrm{H} & -2.798728 & 2.183370 & 5.002801 \\ \mathrm{H} & -2.982589 & 3.749893 & 4.163468\end{array}$

$\left(R_{\mathrm{p}}\right)$-[3.3]pCp-5, 8, 14, 17-tetracarboxamide dimer (Syn_chair_boat) Dihedral $=-138.7^{\circ},-135.1^{\circ}, 34.3^{\circ}, 43.9^{\circ} ;-137.3^{\circ},-142.4^{\circ}, 52.0^{\circ}, 54.5^{\circ}$

Total Energy: -3058.93761031 a.u.

$\begin{array}{lrrr}\mathrm{C} & 4.808592 & -0.421905 & 1.560304 \\ \mathrm{C} & 4.625346 & 0.959309 & 1.469574 \\ \mathrm{C} & 4.865187 & 1.549632 & 0.218071 \\ \mathrm{C} & 5.096431 & 0.759643 & -0.909161 \\ \mathrm{C} & 5.172723 & -0.631618 & -0.836128 \\ \mathrm{C} & 5.113197 & -1.203312 & 0.444915 \\ \mathrm{C} & 4.083358 & 1.723614 & 2.664627 \\ \mathrm{C} & 2.690046 & 1.259684 & 3.138168 \\ \mathrm{C} & 1.523681 & 1.518993 & 2.162946 \\ \mathrm{C} & 1.517657 & 0.623377 & 0.942025 \\ \mathrm{C} & 1.610713 & 1.197304 & -0.326459 \\ \mathrm{C} & 1.849681 & 0.441534 & -1.472577 \\ \mathrm{C} & 2.065080 & -0.943290 & -1.366201 \\ \mathrm{C} & 1.829175 & -1.530003 & -0.125019 \\ \mathrm{C} & 1.527485 & -0.778148 & 1.015782 \\ \mathrm{C} & 1.861487 & 1.115875 & -2.814538 \\ \mathrm{~N} & 2.472144 & 2.307779 & -2.892324 \\ \mathrm{C} & 2.467222 & 3.055065 & -4.135719 \\ \mathrm{C} & 1.291507 & -1.487470 & 2.321367 \\ \mathrm{~N} & 2.072414 & -2.551435 & 2.569328 \\ \mathrm{C} & 1.913856 & -3.308940 & 3.795387 \\ \mathrm{C} & 2.639488 & -1.768032 & -2.500767 \\ \mathrm{C} & 3.991213 & -1.252997 & -3.036014 \\ \mathrm{C} & 5.211652 & -1.451171 & -2.113243 \\ \mathrm{C} & 4.809784 & 3.034863 & -0.002635 \\ \mathrm{~N} & 5.633182 & 3.784358 & 0.758415 \\ \mathrm{C} & 5.671519 & 5.230367 & 0.618351 \\ \mathrm{C} & 5.305143 & -2.676046 & 0.673247 \\ \mathrm{~N} & 6.484920 & -3.187820 & 0.266557 \\ \mathrm{C} & 6.788415 & -4.599153 & 0.433715 \\ \mathrm{O} & 1.310408 & 0.594962 & -3.793086 \\ \mathrm{O} & 0.435387 & -1.118372 & 3.134069 \\ \mathrm{O} & 4.084612 & 3.549948 & -0.855593 \\ \mathrm{O} & 4.459281 & -3.380911 & 1.226160 \\ \mathrm{~N} & -1.572620 & 0.849420 & 3.631311 \\ \mathrm{C} & -1.584633 & 1.564889 & 4.889782 \\ \mathrm{C} & -2.202698 & 1.349154 & 2.554864 \\ \mathrm{O} & -2.807439 & 2.427228 & 2.599498 \\ \mathrm{C} & -2.100417 & 0.530586 & 1.298912 \\ \mathrm{C} & -2.333138 & -0.858948 & 1.270666 \\ \mathrm{C} & -2.078701 & -1.506418 & 0.066662 \\ \mathrm{C} & -1.743253 & -0.827177 & -1.113229 \\ \mathrm{C} & -1.695647 & 0.574301 & -1.117841 \\ \mathrm{C} & -1.814042 & 1.215333 & 0.122207 \\ \mathrm{C} & -2.925621 & -1.640476 & 2.433360 \\ \mathrm{C} & -4.205658 & -1.049538 & 3.068736 \\ & & & \end{array}$




\begin{tabular}{|c|c|c|}
\hline 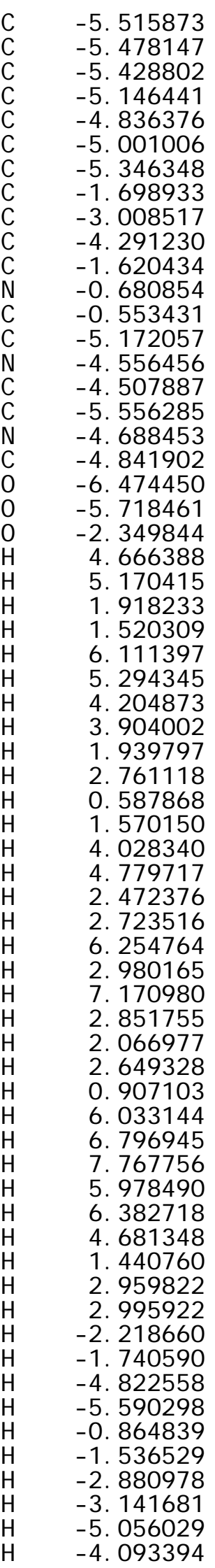 & $\begin{array}{r}-1.261409 \\
-0.605273 \\
0.790528 \\
1.462823 \\
0.732691 \\
-0.649310 \\
-1.324319 \\
1.438910 \\
\text { 2. } 235193 \\
1.399951 \\
-1.699274 \\
-1.400634 \\
-2.179798 \\
2.967605 \\
3.589978 \\
5.035581 \\
-2.817336 \\
-3.519846 \\
-4.952033 \\
-3.361223 \\
3.584588 \\
-2.689920 \\
-0.919712 \\
1.250454 \\
-2.611343 \\
2.279039 \\
-1.186627 \\
-2.517158 \\
-1.787991 \\
-0.195714 \\
-1.795541 \\
-2.801652 \\
1.409775 \\
\text { 2. } 562276 \\
2.792757 \\
1.617606 \\
1.798013 \\
0.198383 \\
3.321049 \\
2.681428 \\
-2.565730 \\
-2.781576 \\
-2.668966 \\
-4.114539 \\
-3.731565 \\
-5.209130 \\
-4.867490 \\
-4.799878 \\
5.508817 \\
5.633479 \\
5.654401 \\
3.261344 \\
2.488393 \\
3.994968 \\
-2.583670 \\
2.302171 \\
-1.228269 \\
1.371077 \\
\text { 2. } 154567 \\
0.843129 \\
2.873985 \\
2.919259 \\
\text { 2. } 067341 \\
\text { - } 623467\end{array}$ & 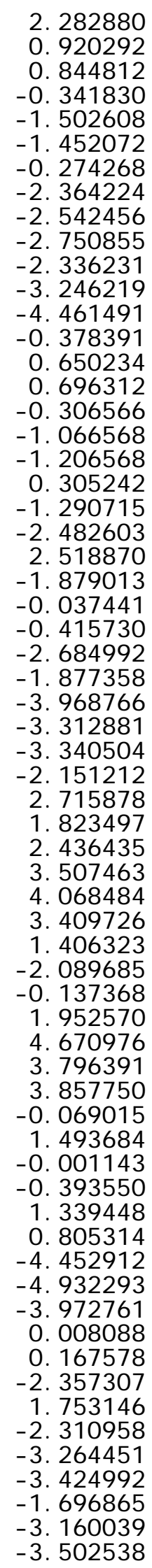 \\
\hline
\end{tabular}




$\begin{array}{rrrr}\mathrm{H} & -5.725592 & -2.328165 & 2.185547 \\ \mathrm{H} & -6.335757 & -0.831967 & 2.872675 \\ \mathrm{H} & -2.168479 & -1.761756 & 3.217258 \\ \mathrm{H} & -3.152228 & -2.652575 & 2.071909 \\ \mathrm{H} & -4.073194 & 0.020770 & 3.264655 \\ \mathrm{H} & -4.331290 & -1.520896 & 4.051705 \\ \mathrm{H} & -0.948215 & 0.053393 & 3.502741 \\ \mathrm{H} & -3.994479 & 3.057902 & 1.316224 \\ \mathrm{H} & -0.061174 & -0.599657 & -3.143210 \\ \mathrm{H} & -3.895654 & -3.069557 & -1.523971 \\ \mathrm{H} & -4.760509 & -5.452059 & -0.235294 \\ \mathrm{H} & -4.058033 & -5.315983 & -1.873136 \\ \mathrm{H} & -5.823375 & -5.195816 & -1.625550 \\ \mathrm{H} & -1.516288 & -2.246386 & -4.976497 \\ \mathrm{H} & -0.217870 & -3.199143 & -4.240946 \\ \mathrm{H} & 0.174150 & -1.680911 & -5.104718 \\ \mathrm{H} & -1.119494 & 2.552207 & 4.788267 \\ \mathrm{H} & -1.037094 & 0.976903 & 5.627964 \\ \mathrm{H} & -2.613494 & 1.712325 & 5.232152 \\ \mathrm{H} & -5.516852 & 5.454541 & 0.759949 \\ \mathrm{H} & -4.035232 & 5.441133 & -0.205842 \\ \mathrm{H} & -3.933971 & 5.331629 & 1.576110\end{array}$




\section{NMR STUDIES}

\section{General Information}

Variable concentration (at $298 \mathrm{~K}$ ) and variable temperature NMR studies were performed on an Inova $500 \mathrm{MHz}$ spectrometer with a Varian $5 \mathrm{~mm}$ conventional probe H1/P31-N15. Cyclohexane- $d_{12}$ was purchased from Sigma-Aldrich. Association constants were calculated using nonlinear curve fitting in Origin 8.5 in accord with a dimerization and isodesmic polymerization association model. ${ }^{6}$ DOSY spectra were recorded by performing a series of pulsed field gradient (PFG) stimulated echo experiments at $263 \mathrm{~K}$ and $298 \mathrm{~K}$, using a bipolar pulse-pair stimulatedecho pulse sequence with a gradient strength of $2 \mathrm{~ms}$ and diffusion delay of $50 \mathrm{~ms}$. DOSY data was processed using Vnmrj software to obtain diffusion coefficients, which are reported as the average values for all major peaks. Contour plots were prepared using the Bayesian DOSY Transform feature in MNova. $\mathrm{CDCl}_{3}$ with $0.01 \%$ TMS was purchased from Cambridge Isotope Laboratories and dried over activated molecular sieves ( $4 \AA)$ prior to use. Cyclohexane- $d_{12}$ was purchased from Sigma-Aldrich.

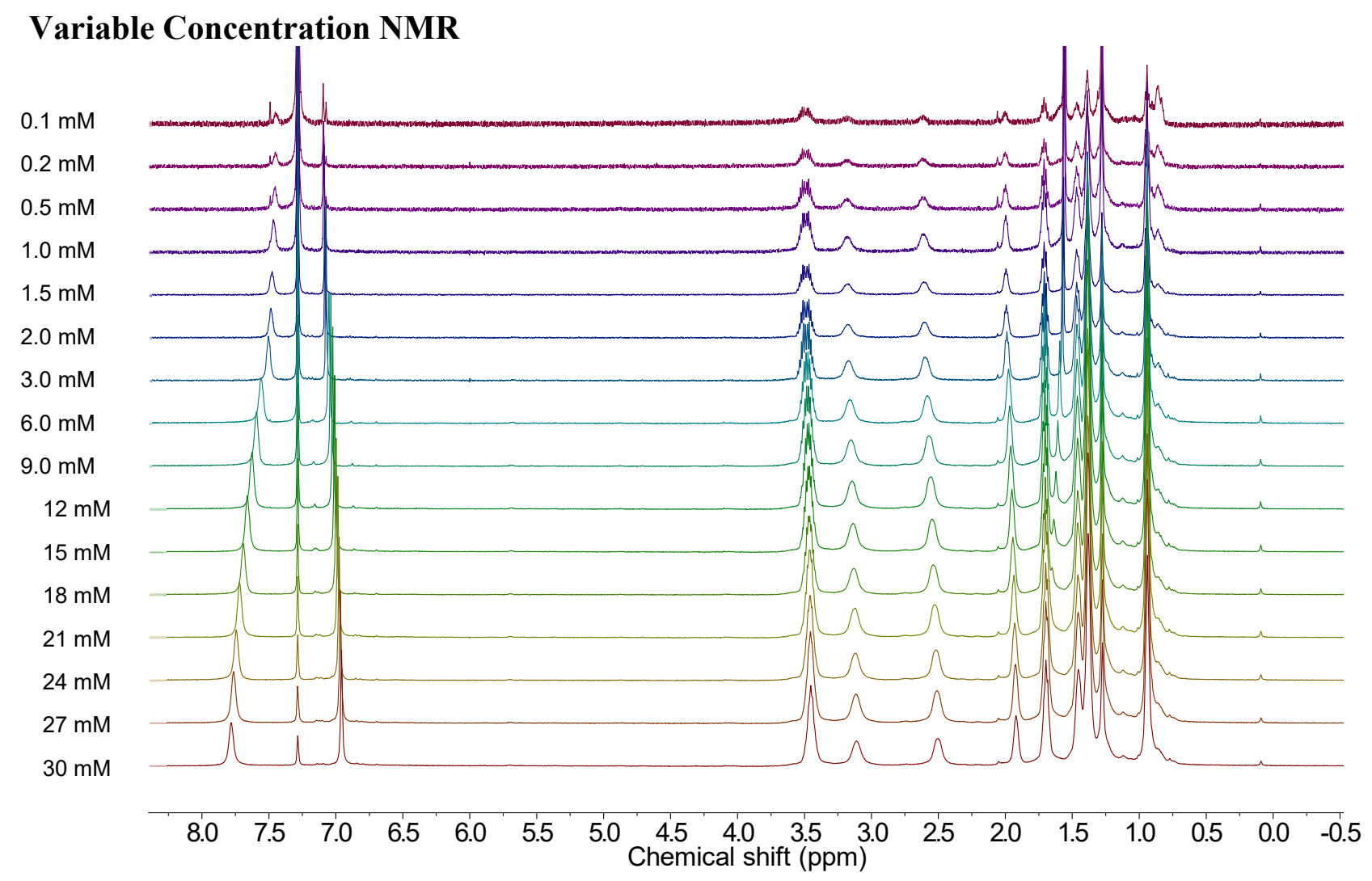

Figure S10. Overlaid 1H NMR spectra of $( \pm)-\mathbf{1 a}\left(0.1-30 \times 10^{-3} \mathrm{M}\right)$ in $\mathrm{CDCl}_{3}$ at $298 \mathrm{~K}$. 


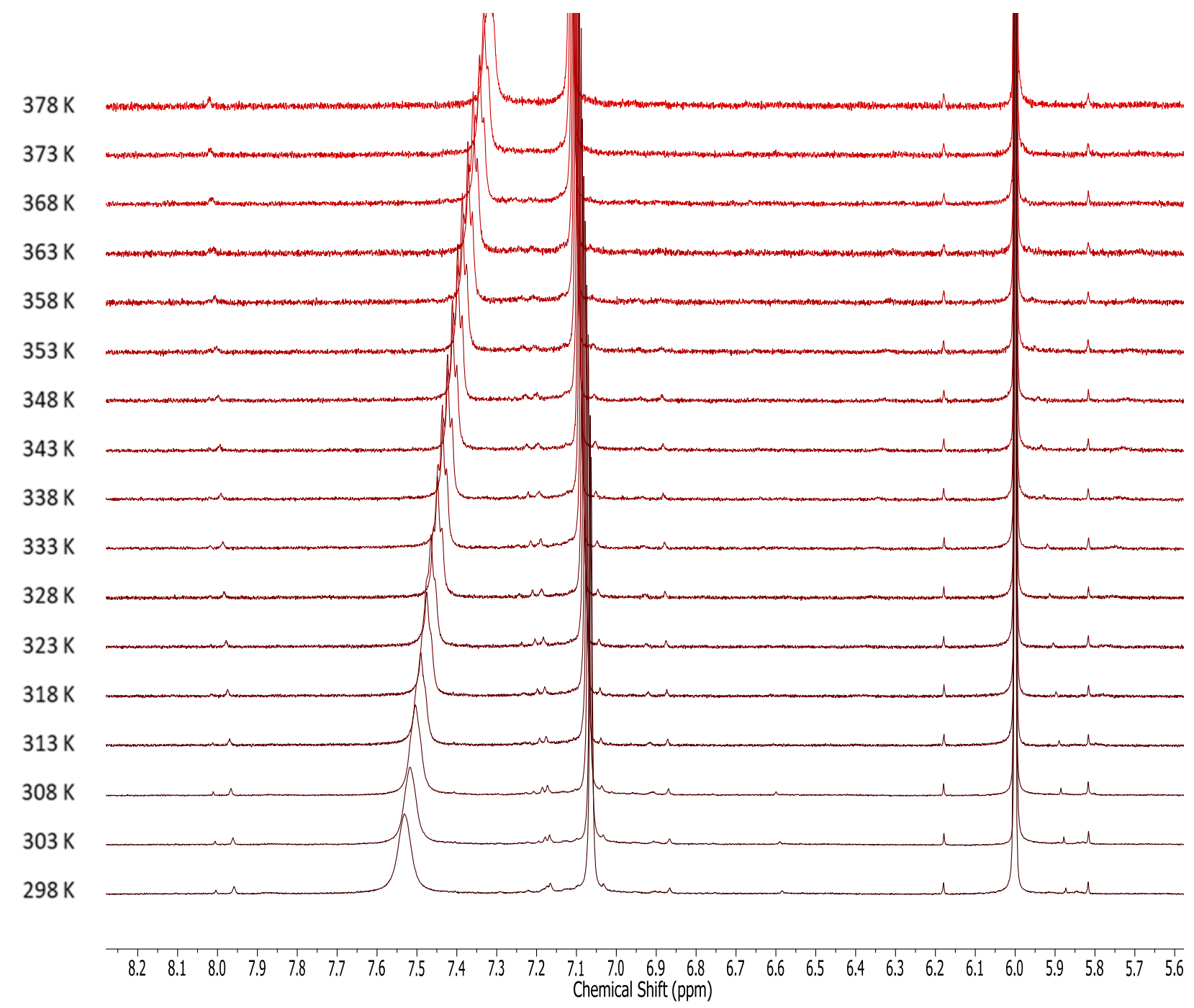

Figure S11. Overlaid ${ }^{1} \mathrm{H}$ NMR spectra of $( \pm)-\mathbf{1 a}\left(10 \times 10^{-3} \mathrm{M}\right)$ in $1,1^{\prime}, 2,2^{\prime}$ tetrachloroethane- $d_{2}$ from 298-378 K. 


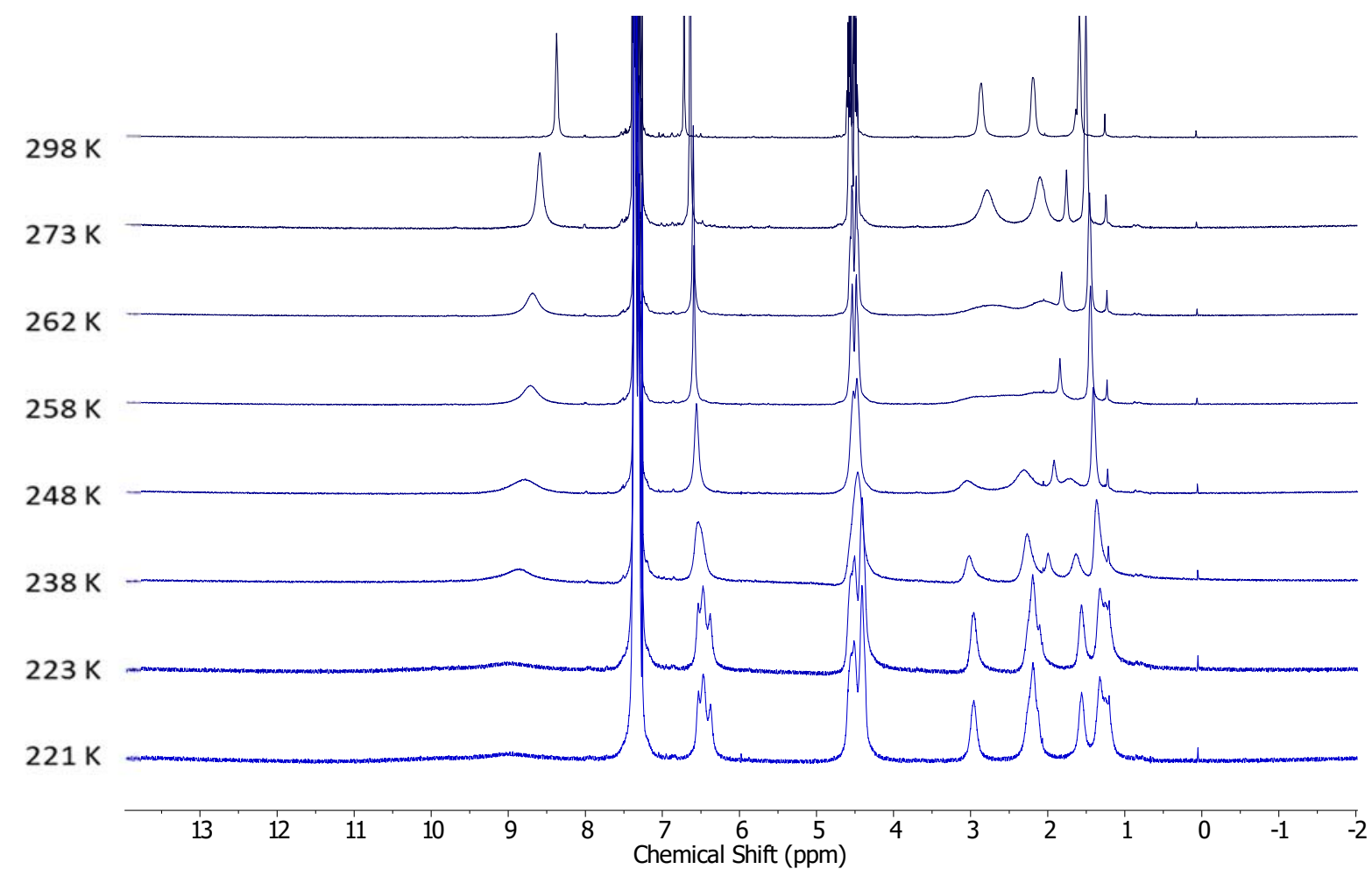

Figure S12. Overlaid ${ }^{1} \mathrm{H}$ NMR spectra of $( \pm)-1 \mathrm{c}\left(26 \times 10^{-3} \mathrm{M}\right)$ in $\mathrm{CDCl}_{3}$ from $298-221 \mathrm{~K}$.

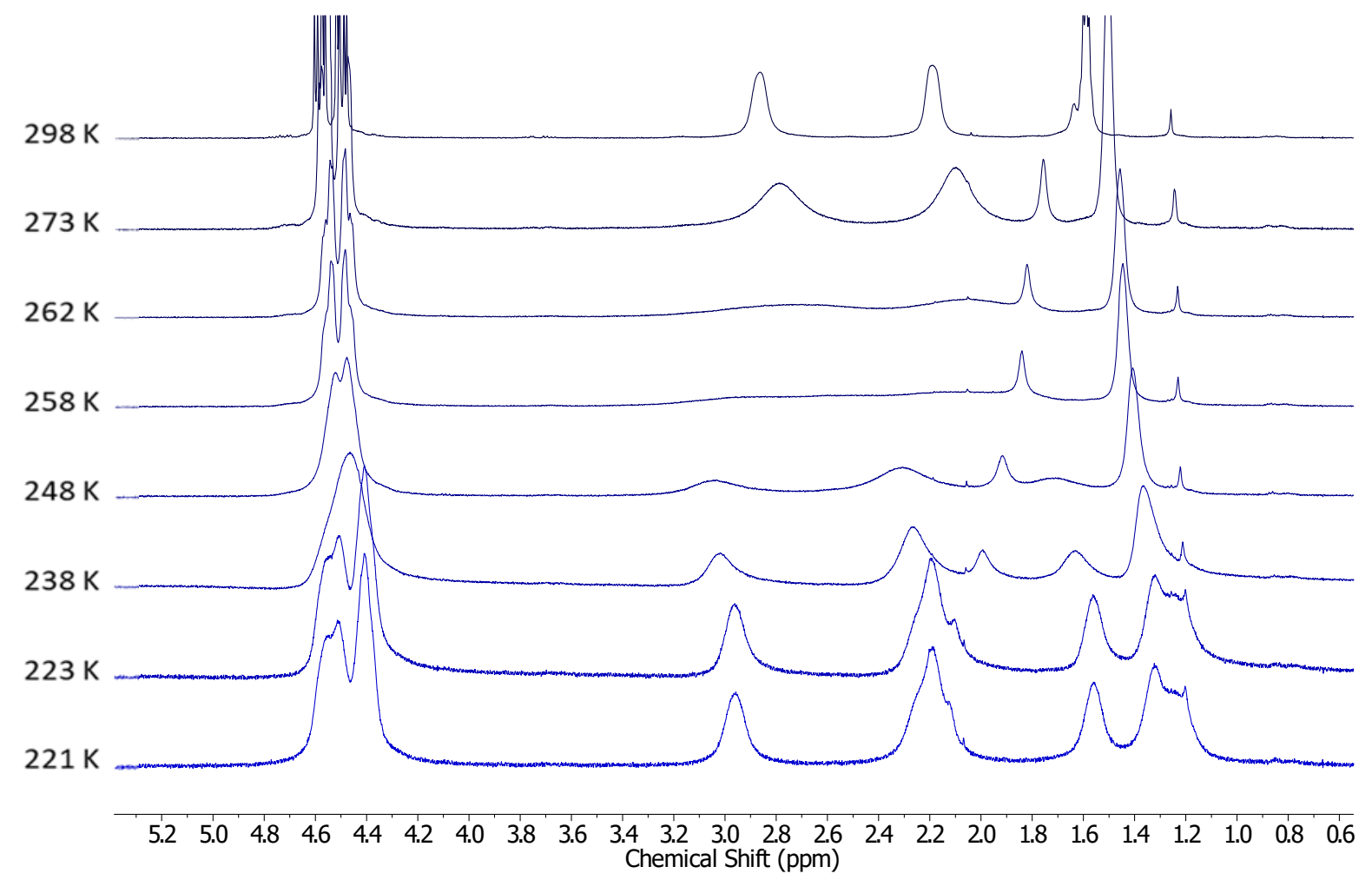

Figure S13. Overlaid ${ }^{1} \mathrm{H}$ NMR spectra of the benzylic region of $( \pm)-1 \mathrm{c}\left(26 \times 10^{-3} \mathrm{M}\right)$ in $\mathrm{CDCl}_{3}$ from $298-221 \mathrm{~K}$. 


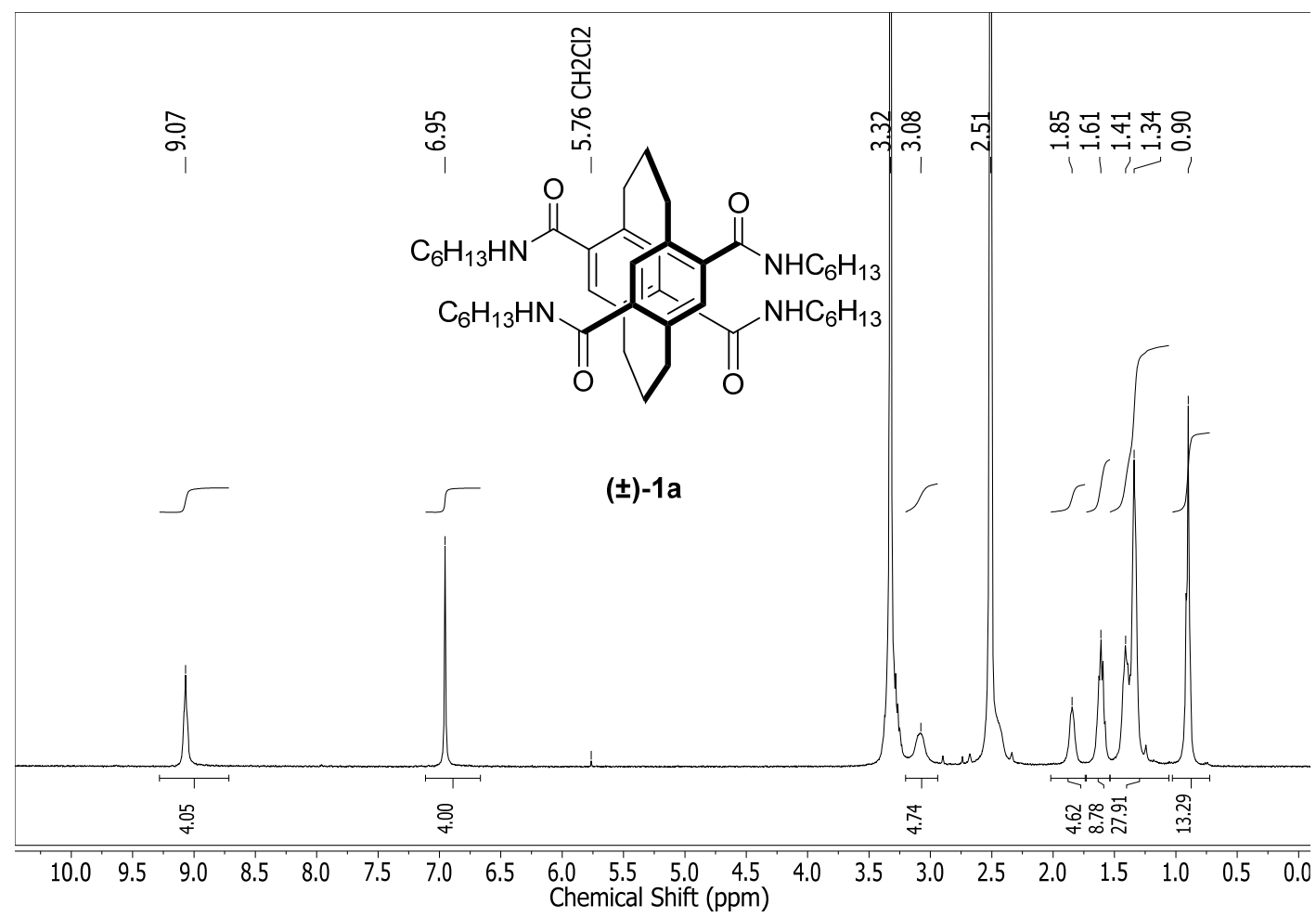

Figure S14. ${ }^{1} \mathrm{H}$ NMR spectra of ( \pm )-1a in DMSO- $d_{6}$ at $298 \mathrm{~K}$

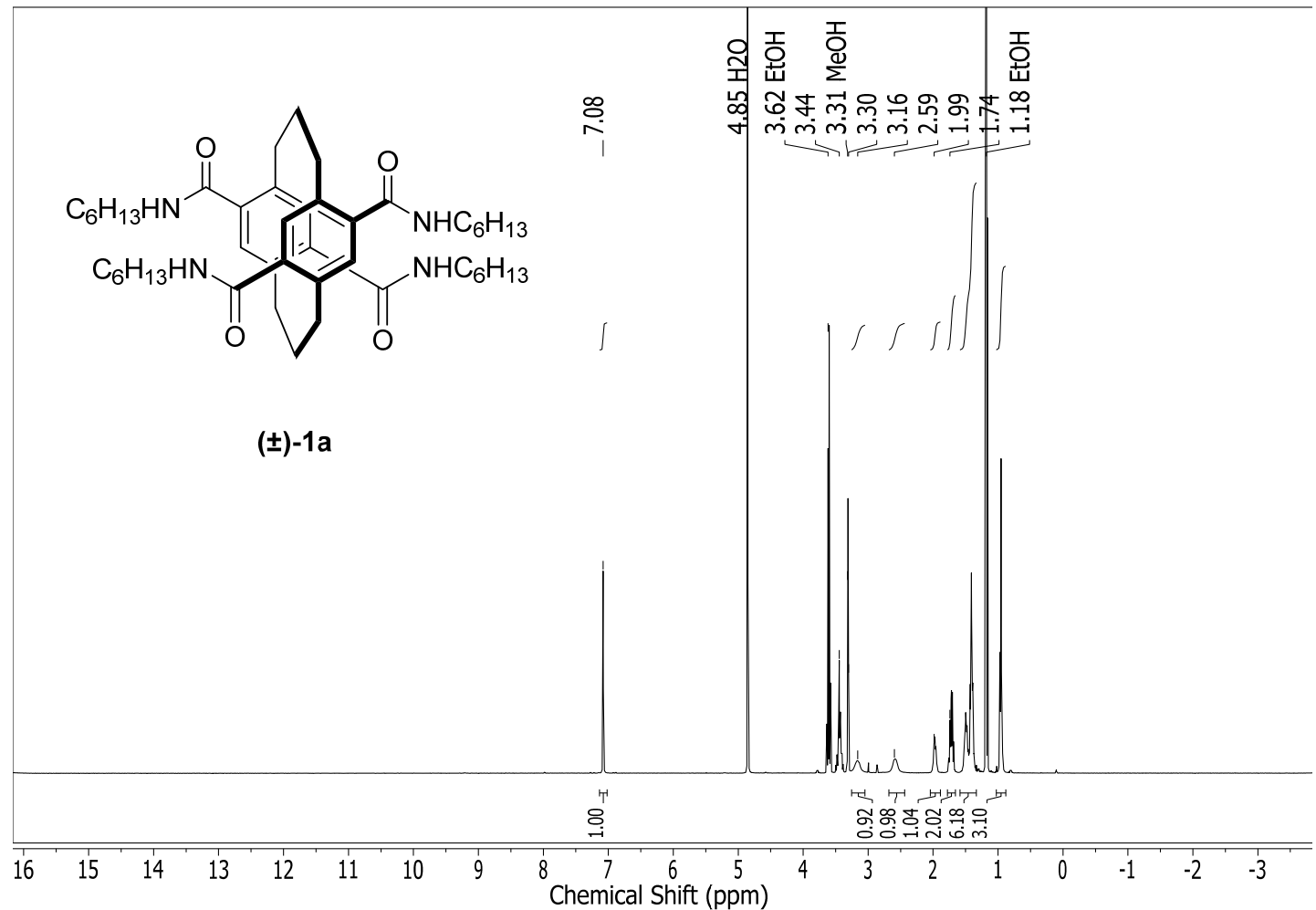

Figure S15. ${ }^{1} \mathrm{H}$ NMR spectra of $( \pm)-1 \mathbf{a}$ in MeOD- $d_{4}$ at $298 \mathrm{~K}$ 


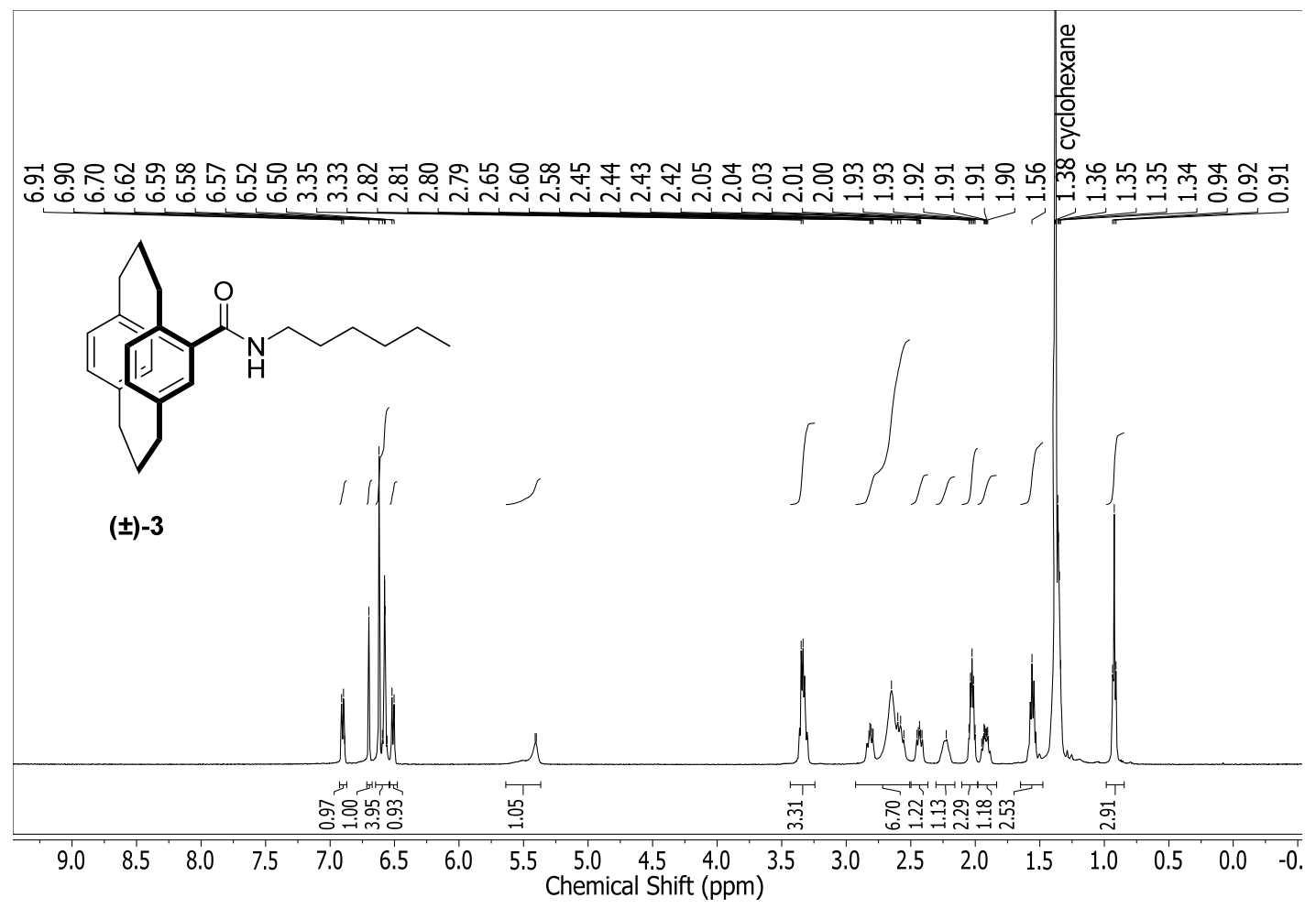

Figure S16. ${ }^{1} \mathrm{H}$ NMR spectra of $( \pm)-3\left(3.0 \times 10^{-2} \mathrm{M}\right)$ in cyclohexane- $d_{12}$ at $298 \mathrm{~K}$.

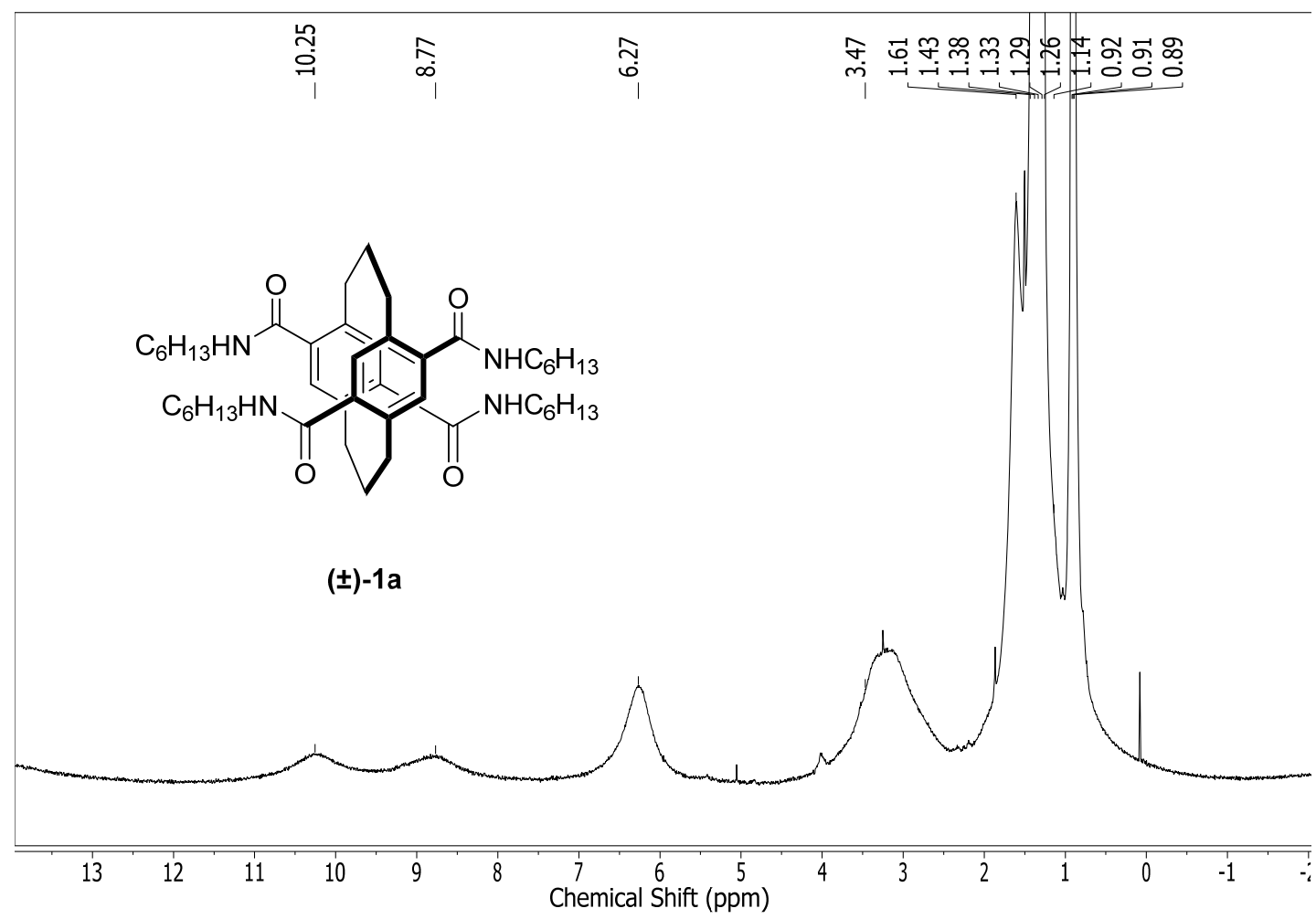

Figure S17. ${ }^{1} \mathrm{H}$ NMR spectra of $( \pm)-1 \mathrm{a}\left(1.0 \times 10^{-2} \mathrm{M}\right)$ in cyclohexane- $d_{12}$ at $298 \mathrm{~K}$. 


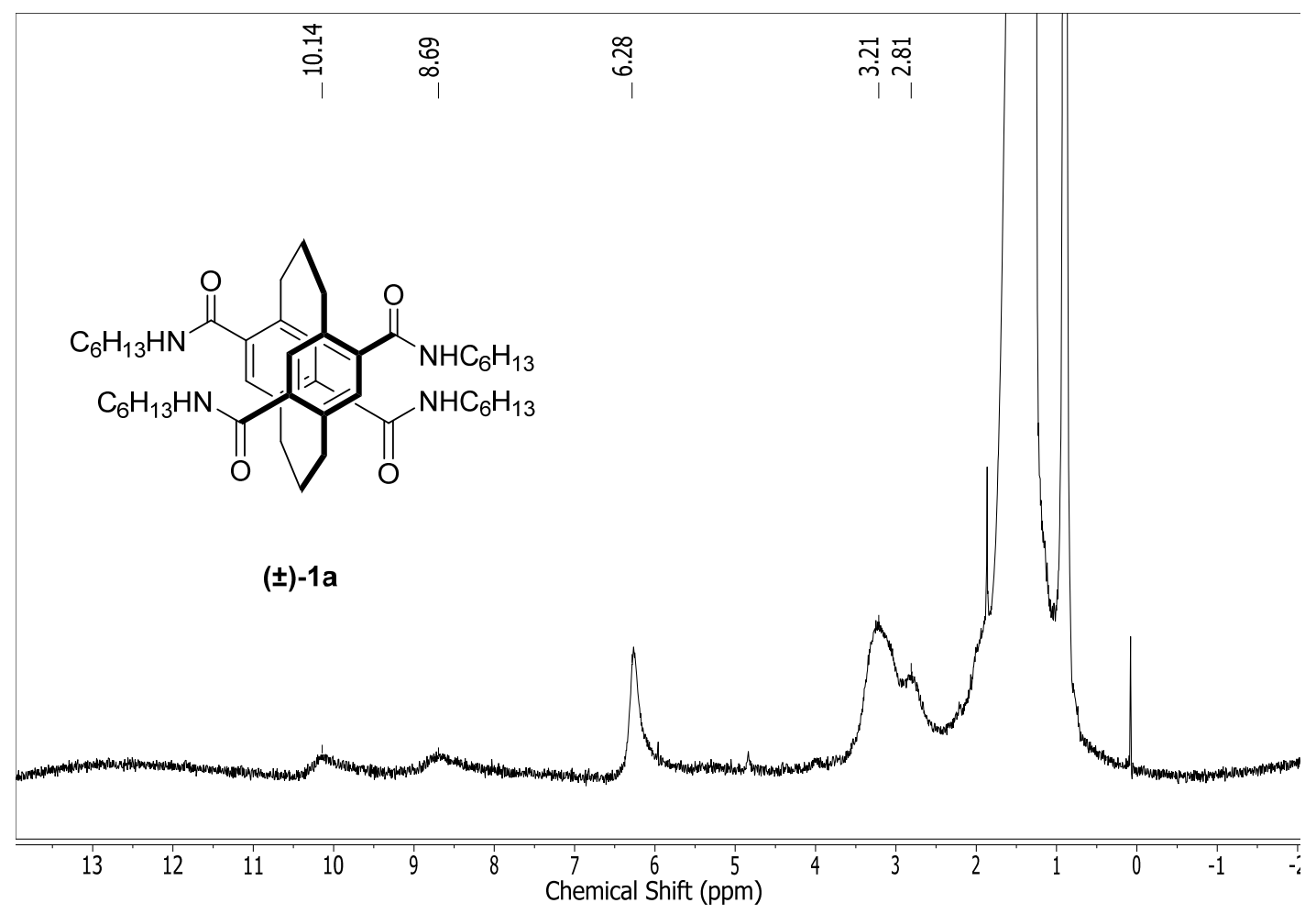

Figure S18. ${ }^{1} \mathrm{H}$ NMR spectra of $( \pm)-1 \mathbf{a}\left(1.0 \times 10^{-3} \mathrm{M}\right)$ in cyclohexane- $d_{12}$ at $298 \mathrm{~K}$.

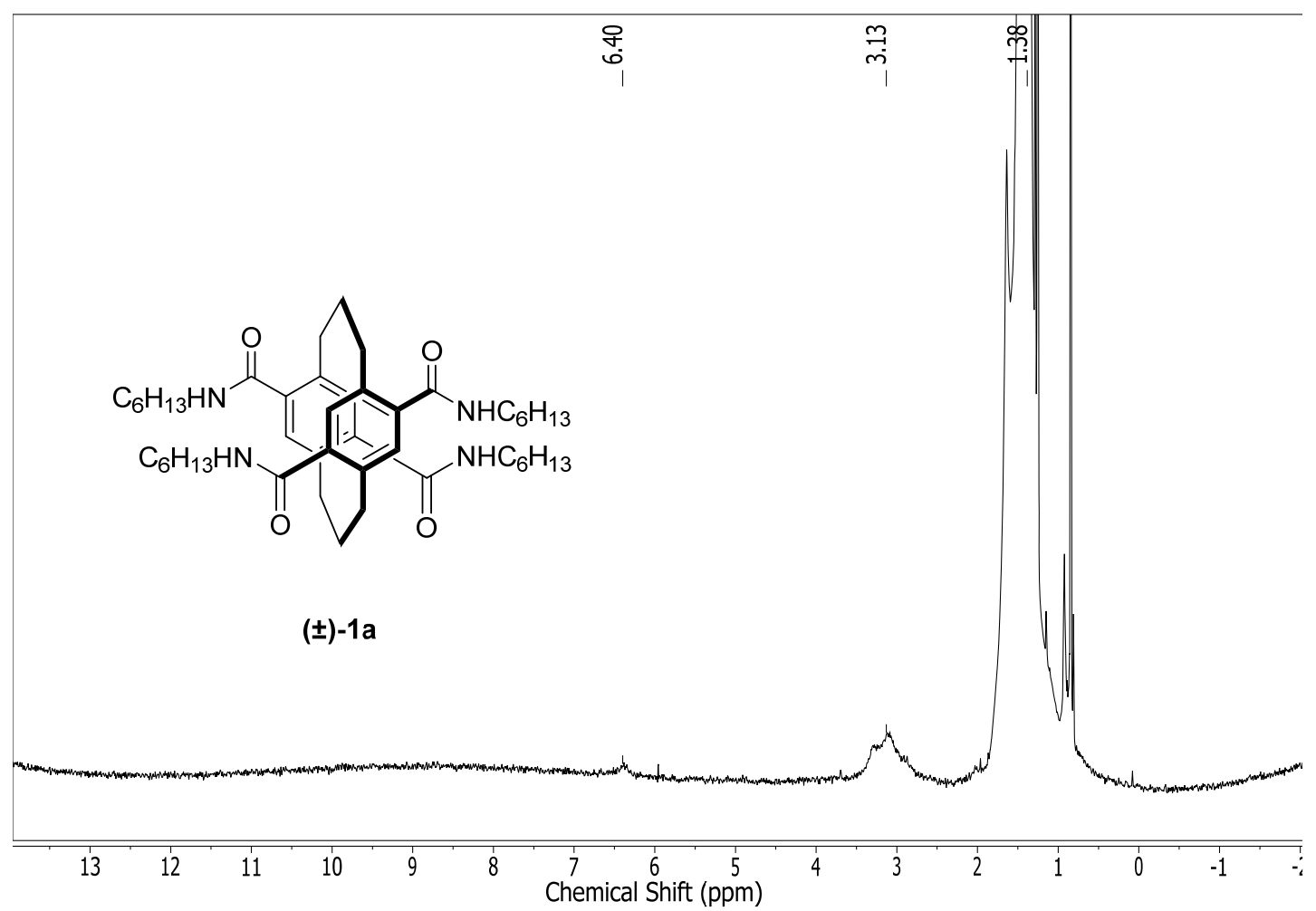

Figure S19. ${ }^{1} \mathrm{H}$ NMR spectra of $( \pm)-1 \mathrm{a}\left(1.0 \times 10^{-4} \mathrm{M}\right)$ in cyclohexane- $d_{12}$ at $298 \mathrm{~K}$. 


\section{Determination of Association Constants}

The concentration-dependent chemical shift data collected for $( \pm)-\mathbf{1 a}$ was fit to a dimerization model to determine $K_{\mathrm{d}}(e q .1)^{7}$ and an isodesmic polymerization model to determine $K_{\mathrm{el}}($ eq. 2$) .{ }^{8} \delta_{\mathrm{obs}}=$ observed chemical shift , $\delta_{\mathrm{m}}=$ chemical shift of monomer (chosen as the lowest concentration), $\delta_{\mathrm{d}}=$ chemical shift of dimer, $\delta_{\mathrm{agg}}=$ chemical shift of aggregate, $c_{\mathrm{t}}=$ total concentration, $K_{\mathrm{d}}=$ dimerization equilibrium constant, $K_{\mathrm{el}}=$ elongation equilibrium constant.

$$
\begin{gathered}
\delta_{o b s}=\delta_{d}+\left(\delta_{m}-\delta_{d}\right)\left[\frac{-1+\sqrt{1+8 K_{d} C_{t}}}{4 K_{d} C_{t}}\right] \\
\delta_{o b s}=\delta_{m}+\left(\delta_{a g g}-\delta_{m}\right)\left[1+\frac{1-\sqrt{4 K_{e l} C_{t}+1}}{2 K_{e l} C_{t}}\right]
\end{gathered}
$$

a.

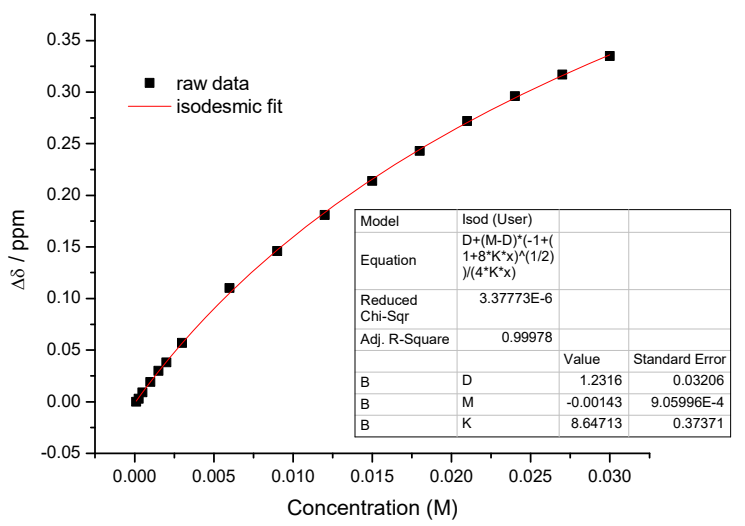

c.

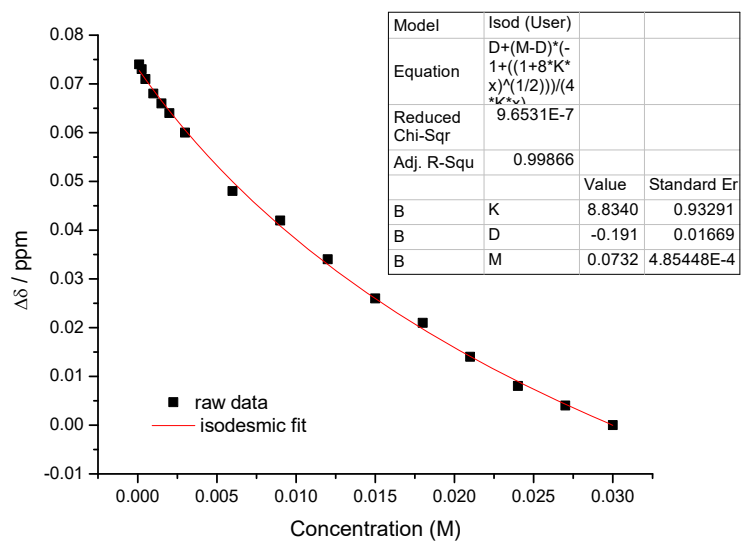

b.

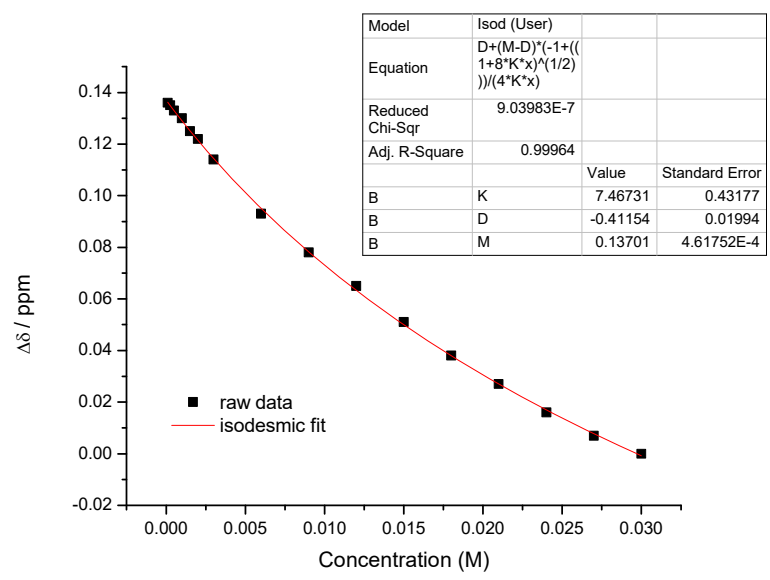

d.

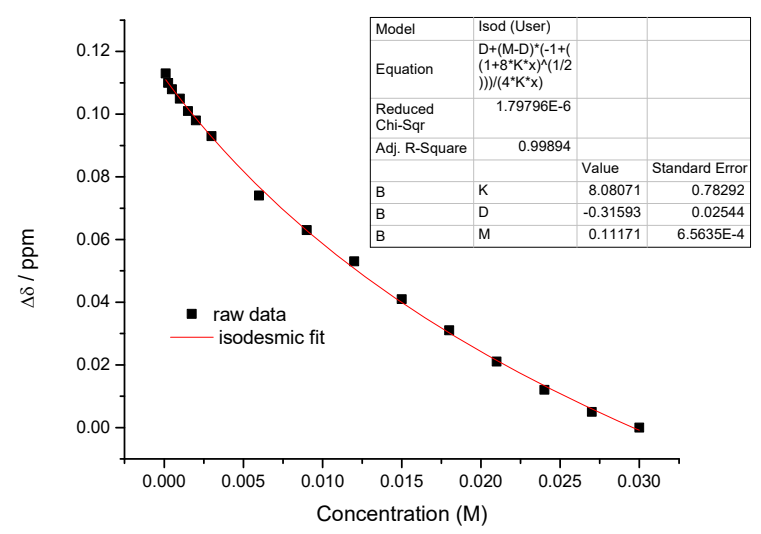

Figure S20. Determination of dimerization constant $K_{\mathrm{d}}$ : Non-linear curve fitting of concentration dependent chemical shifts of $( \pm)-\mathbf{1 a}(\mathrm{a})$ amido $\mathrm{N}-\mathrm{H}$, (b) aromatic $\mathrm{C}-\mathrm{H}$, and $(\mathrm{c}, \mathrm{d})$ bridge protons in $\mathrm{CDCl}_{3}$ using a dimerization model. 
a.

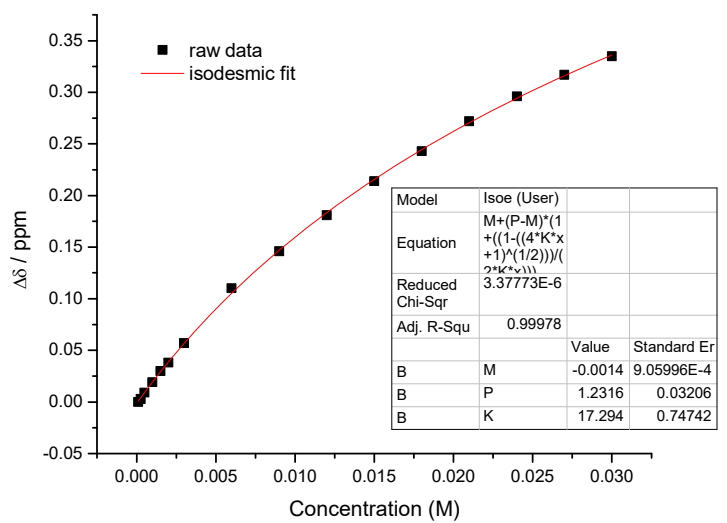

c.

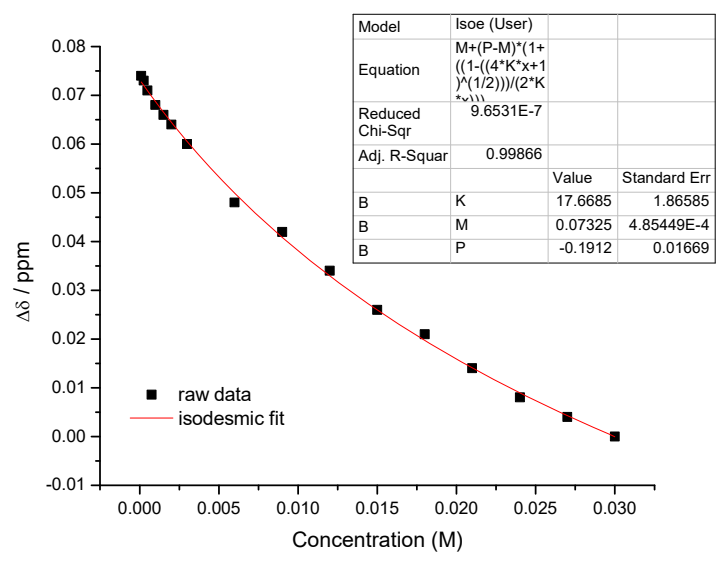

b.

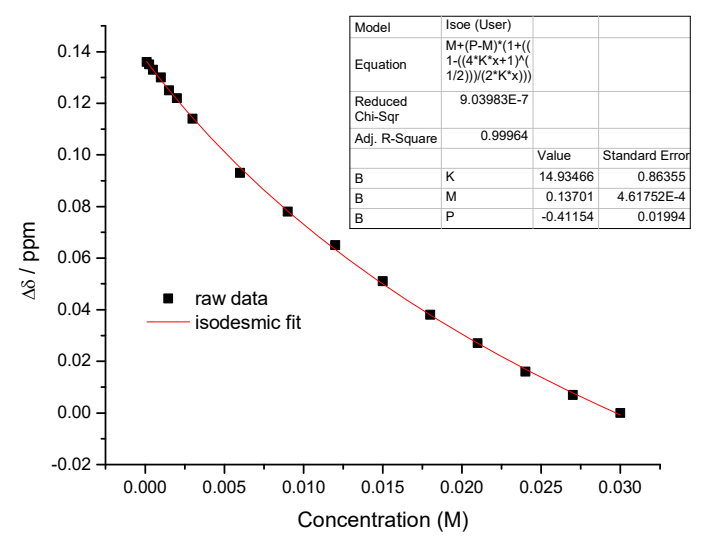

d.

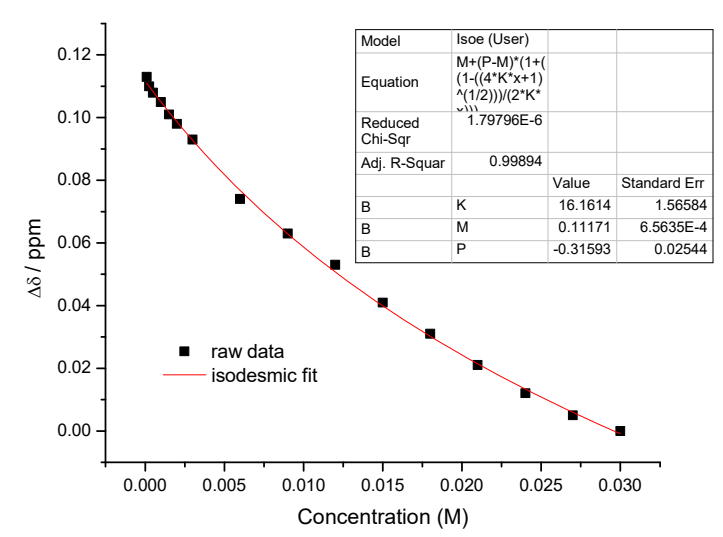

Figure S21. Determination of elongation constant $K_{\mathrm{el}}$ : Non-linear curve fitting of concentration dependent chemical shifts of $( \pm)-1$ a (a) amido $\mathrm{N}-\mathrm{H}$, (b) aromatic $\mathrm{C}-\mathrm{H}$, and (c,d) bridge protons in $\mathrm{CDCl}_{3}$ using a isodesmic model.

Table S2. Association constant determined for each ${ }^{1} \mathrm{H}$ NMR resonance.

\begin{tabular}{|l|c|c|}
\hline Resonance & $\boldsymbol{K}_{\mathbf{d}}\left(\mathbf{M}^{-\mathbf{1}}\right)$ & $\boldsymbol{K}_{\mathbf{e}}\left(\mathbf{M}^{-\mathbf{1}}\right)$ \\
\hline Amido $(\mathrm{N}-\mathrm{H})$ & $9 \pm 1$ & $17 \pm 1$ \\
\hline Aromatic $(\mathrm{C}-\mathrm{H})$ & $8 \pm 1$ & $15 \pm 1$ \\
\hline Bridge $\left(\mathrm{H}_{\mathrm{a}}\right)$ & $9 \pm 1$ & $18 \pm 2$ \\
\hline Bridge $\left(\mathrm{H}_{\mathrm{b}}\right)$ & $8 \pm 1$ & $16 \pm 2$ \\
\hline
\end{tabular}


DOSY NMR Studies
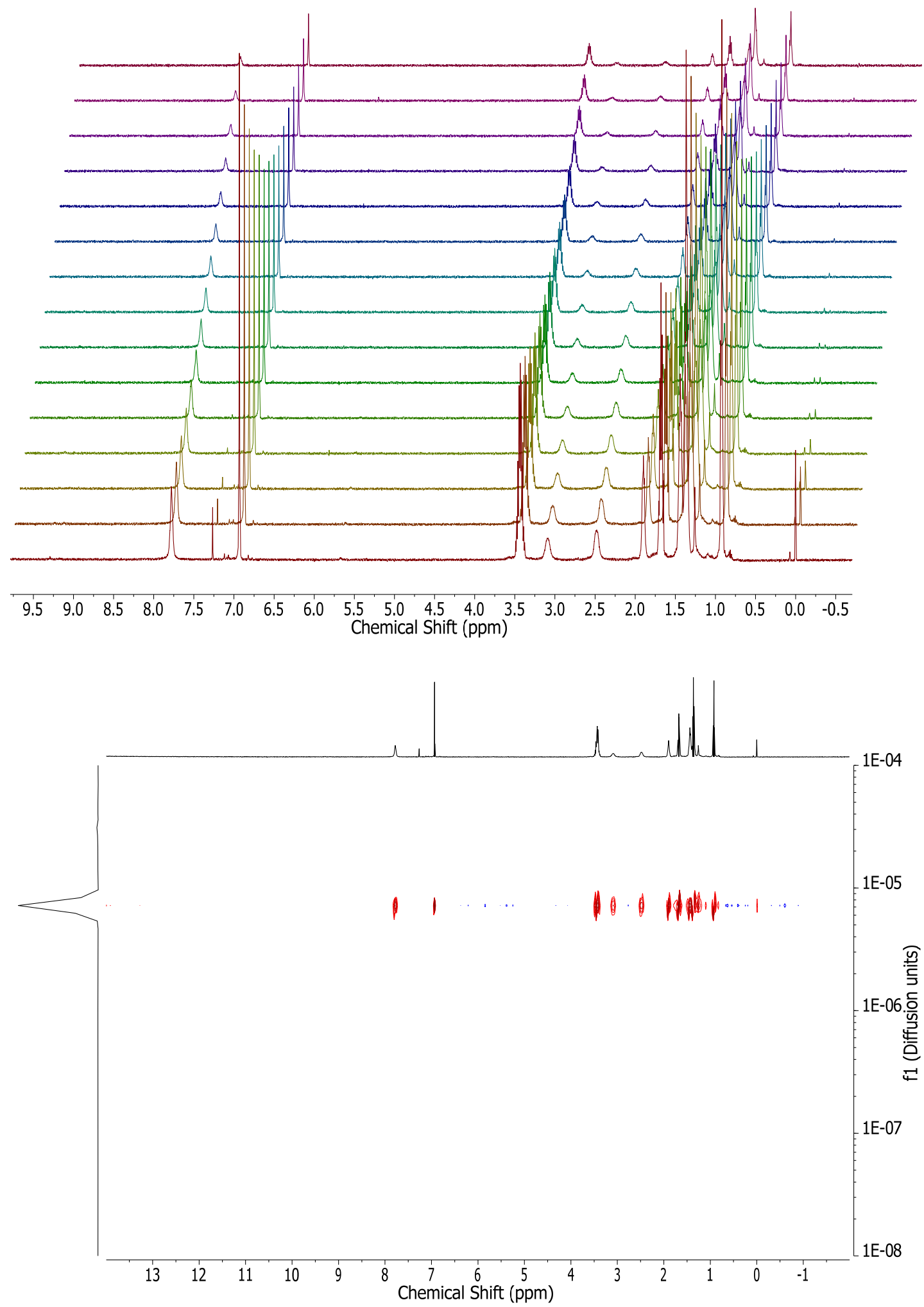

Figure S22. Arrayed DOSY ${ }^{1} \mathrm{H}$ NMR spectra (top) and respective diffusion contour plot of $( \pm)-1 \mathrm{a}\left(30 \times 10^{-3} \mathrm{M}\right.$ in $\mathrm{CDCl}_{3}$ at $\left.298 \mathrm{~K}\right)$. 


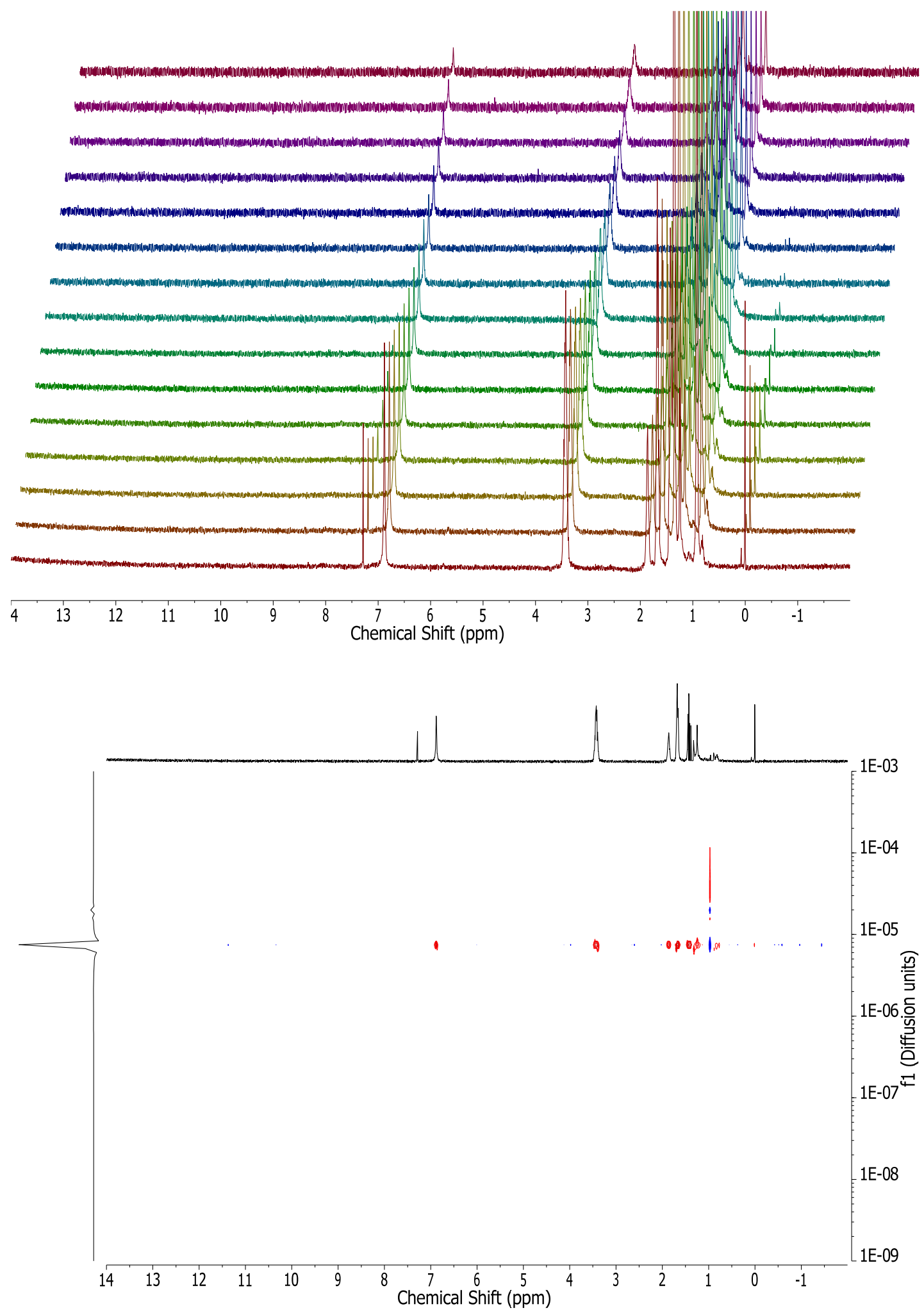

Figure S23. Arrayed DOSY ${ }^{1} \mathrm{H}$ NMR spectra (top) and respective diffusion contour plot of ( \pm )-1a $\left(30 \times 10^{-3} \mathrm{M}\right.$ in $\mathrm{CDCl}_{3}$ at $\left.263 \mathrm{~K}\right)$. 


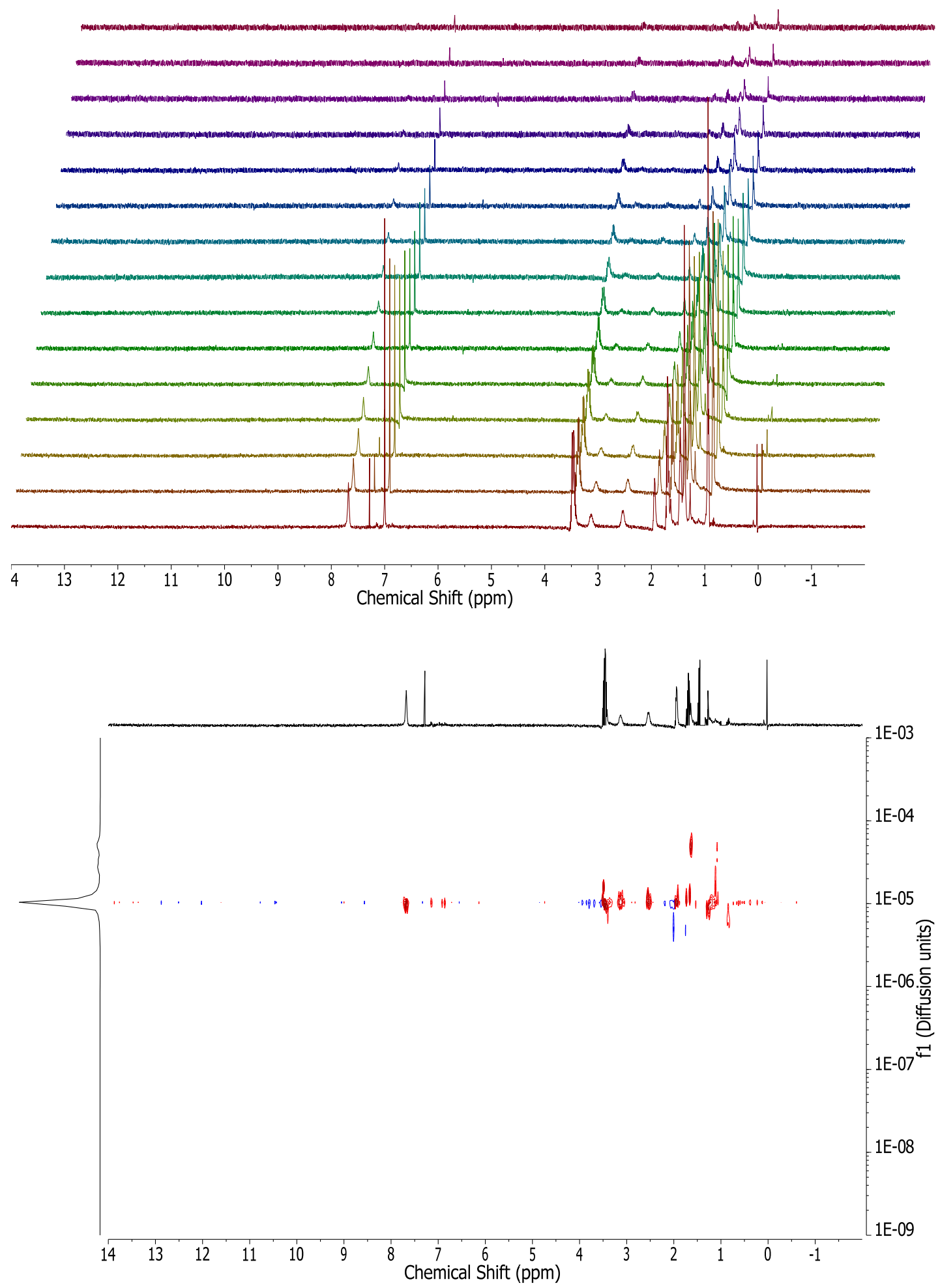

Figure S24. Arrayed DOSY ${ }^{1} \mathrm{H}$ NMR spectra (top) and respective diffusion contour plot of $( \pm)-1 \mathrm{a}\left(15 \times 10^{-3} \mathrm{M}\right.$ in $\mathrm{CDCl}_{3}$ at $\left.298 \mathrm{~K}\right)$. 


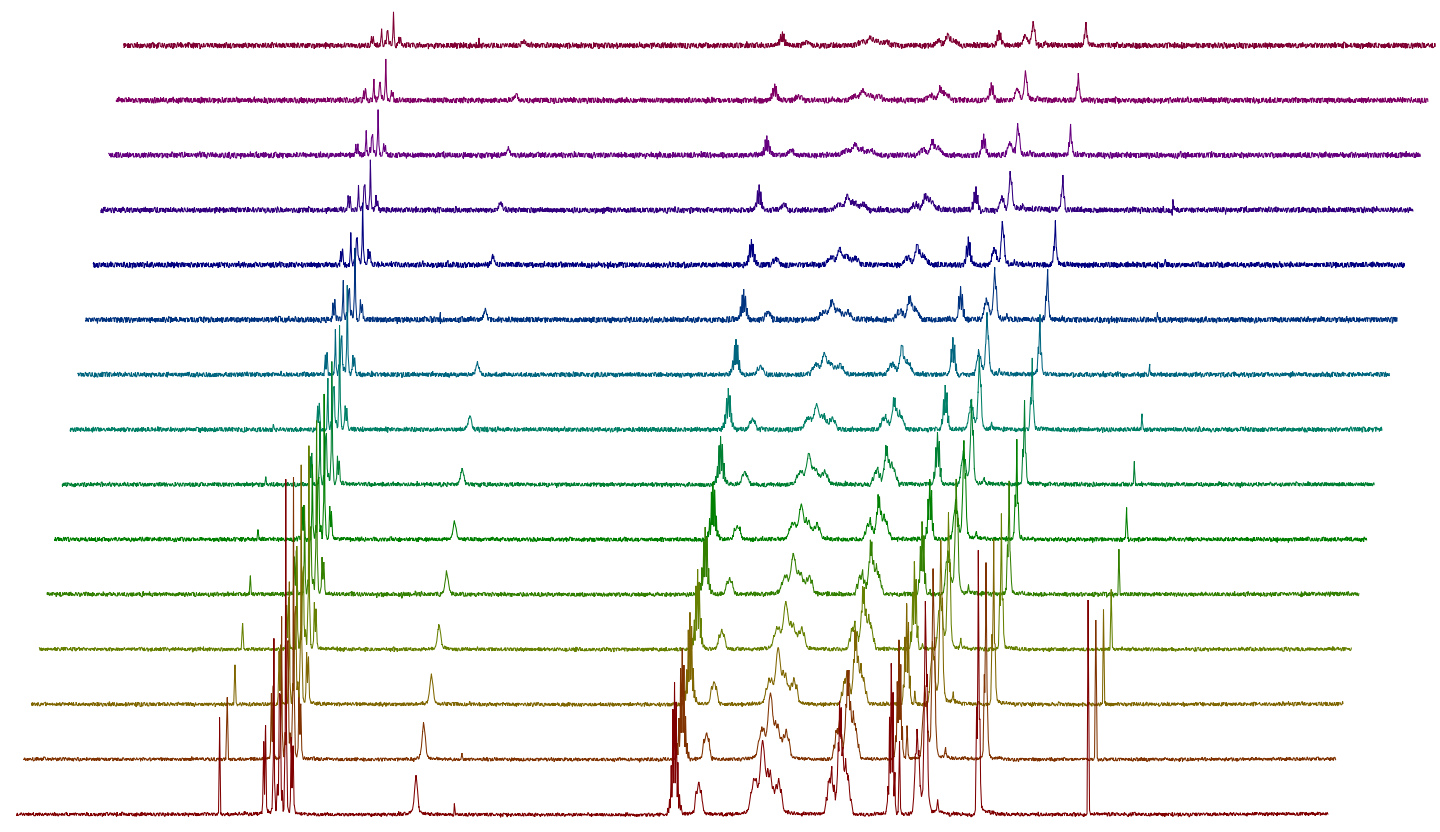

$\begin{array}{llllllllllllllllllllll}8.5 & 8.0 & 7.5 & 7.0 & 6.5 & 6.0 & 5.5 & 5.0 & 4.5 & 4.0 & 3.5 & 3.0 & 2.5 & 2.0 & 1.5 & 1.0 & 0.5 & 0.0 & -0.5 & -1.0 & -1.5\end{array}$ Chemical Shift (ppm)

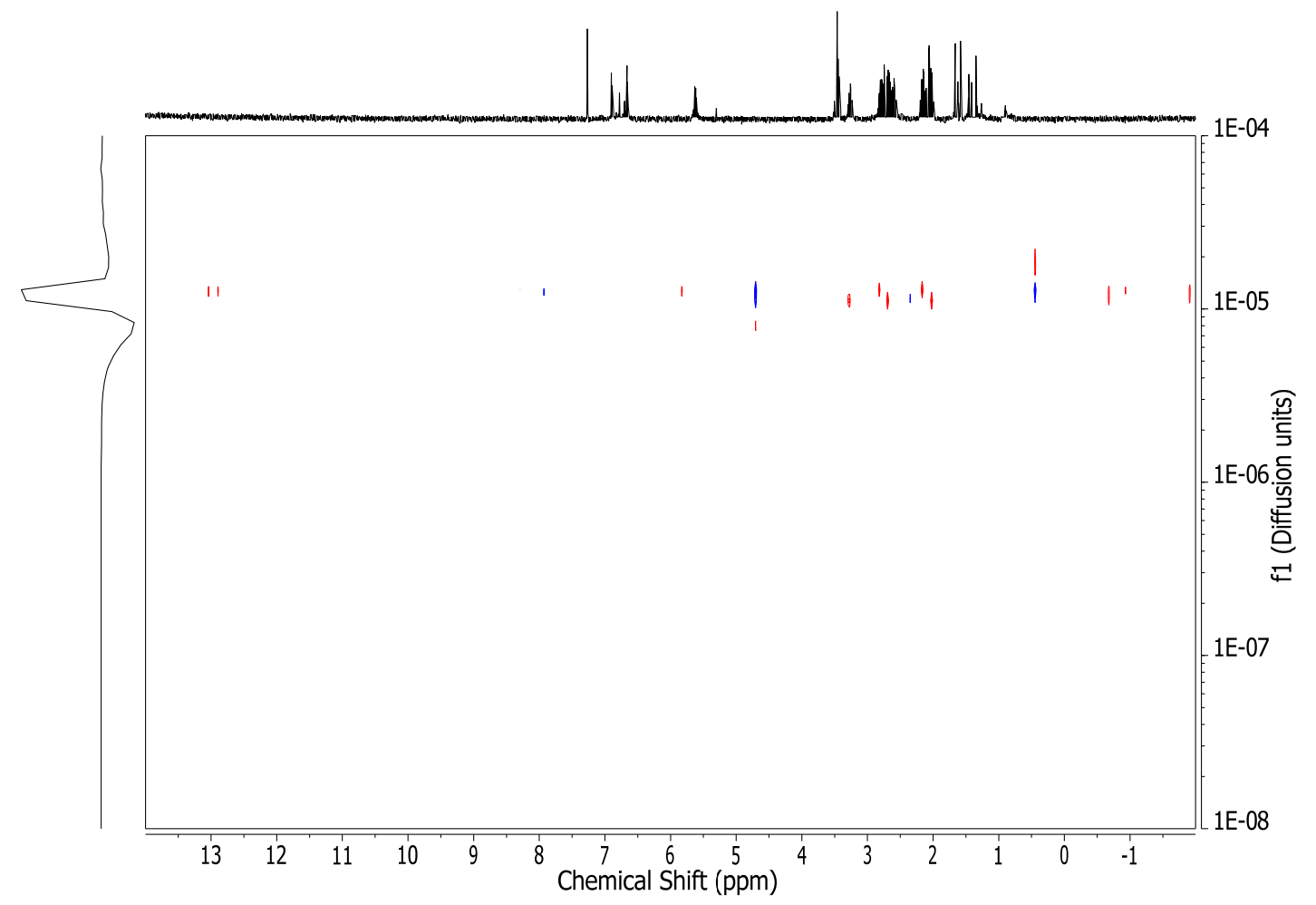

Figure S25. Arrayed DOSY ${ }^{1} \mathrm{H}$ NMR spectra (top) and respective diffusion contour plot of $( \pm)-3\left(30 \times 10^{-3} \mathrm{M}\right.$ in $\mathrm{CDCl}_{3}$ at $298 \mathrm{~K}$ ). 
Table S3. Summary of NMR DOSY data. Data for ( \pm )-2a from ref. 4.

\begin{tabular}{cccc}
\hline Conc. $(\mathbf{m M})$ & Temp $(\mathbf{K})$ & $\boldsymbol{D}_{1 a}\left(10^{-10} \mathrm{~m}^{2} \mathrm{~s}^{-1}\right)$ & $\boldsymbol{D}_{T M S}\left(10^{-10} \mathrm{~m}^{2} \mathrm{~s}^{-1}\right)$ \\
\hline $\mathbf{(})-\mathbf{1 a}$ & & & \\
15 & 298 & $6.7 \pm 0.3$ & $15.5 \pm 2.6$ \\
30 & 298 & $6.0 \pm 0.1$ & $20.3 \pm 2.2$ \\
30 & 263 & $4.0 \pm 0.2$ & $12.5 \pm 0.5$ \\
$( \pm)-2 \mathrm{a}$ & & & \\
1 & 298 & $6.69 \pm 0.09$ & $22.09 \pm 0.09$ \\
15 & 298 & $5.54 \pm 0.05$ & $20.36 \pm 0.05$ \\
30 & 298 & $5.07 \pm 0.05$ & $19.38 \pm 0.08$ \\
30 & 263 & $2.68 \pm 0.03$ & $12.92 \pm 0.3$ \\
$( \pm)-3$ & & & \\
30 & 298 & $6.7 \pm 0.1$ & $16.9 \pm 1.3$ \\
\hline
\end{tabular}




\section{X-RAY CRYSTALLOGRAPHY}

X-Ray experimental: X-Ray Intensity data were collected at $100 \mathrm{~K}$ on a Bruker DUO diffractometer using MoKa radiation $(1=0.71073 \AA)$ and an APEXII CCD area detector.

Raw data frames were read by program $\mathrm{SAINT}^{1}$ and integrated using $3 \mathrm{D}$ profiling algorithms. The resulting data were reduced to produce hkl reflections and their intensities and estimated standard deviations. The data were corrected for Lorentz and polarization effects and numerical absorption corrections were applied based on indexed and measured faces.

The structure was solved and refined in SHELXTL2014, using full-matrix least-squares refinement. The non-H atoms were refined with anisotropic thermal parameters and all of the $\mathrm{H}$ atoms were calculated in idealized positions and refined riding on their parent atoms. The asymmetric unit consists of three molecules and an ethyl acetate solvent molecule which after refining its site occupation factor was fixed at 0.85 . The parallel aromatic rings are link by $-\mathrm{CH} 2-\mathrm{CH} 2-$ $\mathrm{CH} 2$ chains and three of them are disordered. They are $\mathrm{C} 11 \mathrm{~b}, \mathrm{C} 2 \mathrm{c}$ and $\mathrm{C} 11 \mathrm{c}$. In each case the site occupations sites were refined and the parts included the protons on the adjacent $\mathrm{CH} 2$ units. In the final cycle of refinement, 29732 reflections (of which 16917 are observed with $\mathrm{I}>2 \mathrm{~s}(\mathrm{I})$ ) were used to refine 1670 parameters and the resulting $\mathrm{R}_{1}$, wR $\mathrm{R}_{2}$ and $\mathrm{S}$ (goodness of fit) were 5.04\%, $12.98 \%$ and 0.935 , respectively. The refinement was carried out by minimizing the $\mathrm{wR}_{2}$ function using $\mathrm{F}^{2}$ rather than $\mathrm{F}$ values. $\mathrm{R}_{1}$ is calculated to provide a reference to the conventional $\mathrm{R}$ value but its function is not minimized.

SHELXTL2014 (2014). Bruker-AXS, Madison, Wisconsin, USA. 


\section{Crystal data and structure refinement for when $4 .{ }^{9}$}

Identification code

Empirical formula

Formula weight

Temperature

Wavelength

Crystal system

Space group

Unit cell dimensions

Volume

Z

Density (calculated)

Absorption coefficient

$\mathrm{F}(000)$

Crystal size

Theta range for data collection

Index ranges

Reflections collected

Independent reflections

Completeness to theta $=25.242^{\circ}$

Absorption correction

Max. and min. transmission

Refinement method

Data / restraints / parameters

Goodness-of-fit on $\mathrm{F}^{2}$

Final R indices $[\mathrm{I}>2 \operatorname{sigma}(\mathrm{I})]$

$\mathrm{R}$ indices (all data)

Largest diff. peak and hole

$\mathrm{R} 1=\mathrm{a}\left(|| \mathrm{F}_{\mathrm{O}}|-| \mathrm{F}_{\mathrm{C}}||\right) / \stackrel{\mathrm{a}}{\left|\mathrm{F}_{\mathrm{O}}\right|}$ when 4

C51.13 H50.27 N4 O4.57

793.88

100(2) K

$0.71073 \AA$

Triclinic

P-1

$$
\begin{array}{ll}
\mathrm{a}=14.0592(4) \AA & \mathrm{a}=107.4634(6)^{\circ} . \\
\mathrm{b}=21.5785(6) \AA & \mathrm{b}=97.4559(7)^{\circ} . \\
\mathrm{c}=22.5867(7) \AA & \mathrm{g}=91.0639(6)^{\circ} .
\end{array}
$$

$6469.2(3) \AA^{3}$

6

$1.223 \mathrm{Mg} / \mathrm{m}^{3}$

$0.078 \mathrm{~mm}^{-1}$

2530

$0.442 \times 0.152 \times 0.086 \mathrm{~mm}^{3}$

1.463 to $27.500^{\circ}$.

$-18 \leq \mathrm{h} \leq 18,-28 \leq \mathrm{k} \leq 28,-29 \leq \mathrm{l} \leq 29$

118340

$29732[\mathrm{R}($ int $)=0.0528]$

$100.0 \%$

Integration

0.9943 and 0.9789

Full-matrix least-squares on $\mathrm{F}^{2}$

29732 / 0 / 1670

0.935

$\mathrm{R} 1=0.0504, \mathrm{wR} 2=0.1299$ [16917]

$\mathrm{R} 1=0.1021, \mathrm{wR} 2=0.1464$

0.548 and -0.326 e. $\AA^{-3}$

$$
\begin{aligned}
& \mathrm{wR} 2=\left[\mathrm{a}\left[\mathrm{w}\left(\mathrm{F}_{\mathrm{o}}^{2}-\mathrm{Fc}^{2}\right)^{2}\right] / \mathrm{a}\left[\mathrm{w}\left(\mathrm{F}_{\mathrm{o}}^{2}\right)^{2}\right]\right]^{1 / 2} \\
& \mathrm{~S}=\left[\mathrm{a}\left[\mathrm{w}\left(\mathrm{Fo}_{\mathrm{o}}^{2}-\mathrm{Fc}^{2}\right)^{2}\right] /(\mathrm{n}-\mathrm{p})\right]^{1 / 2} \\
& \mathrm{w}=1 /\left[\mathrm{s}^{2}\left(\mathrm{Fo}_{\mathrm{o}}^{2}\right)+\left(\mathrm{m}^{*} \mathrm{p}\right)^{2}+\mathrm{n}^{*} \mathrm{p}\right], \mathrm{p}=\left[\max \left(\mathrm{Fo}_{\mathrm{o}}^{2}, 0\right)+2^{*} \mathrm{Fc}^{2}\right] / 3, \mathrm{~m} \& \mathrm{n} \text { are constants. }
\end{aligned}
$$




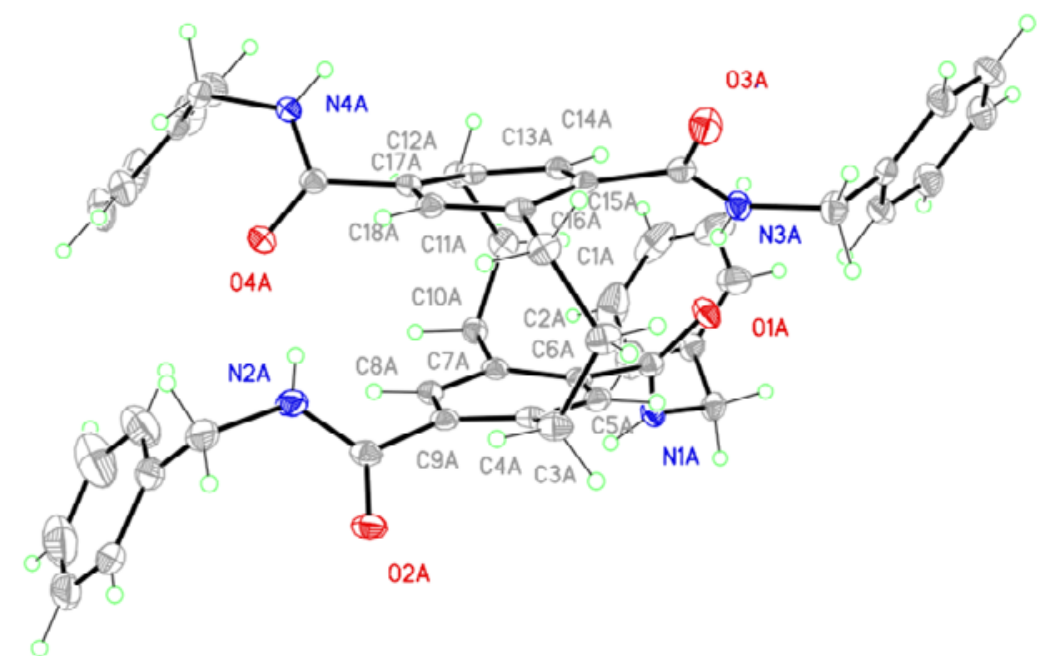

Figure S26. Thermal displacement ellipsoids (50\%) show one of the molecules $\left(\boldsymbol{S}_{p}\right)$-1c in the asymmetric unit

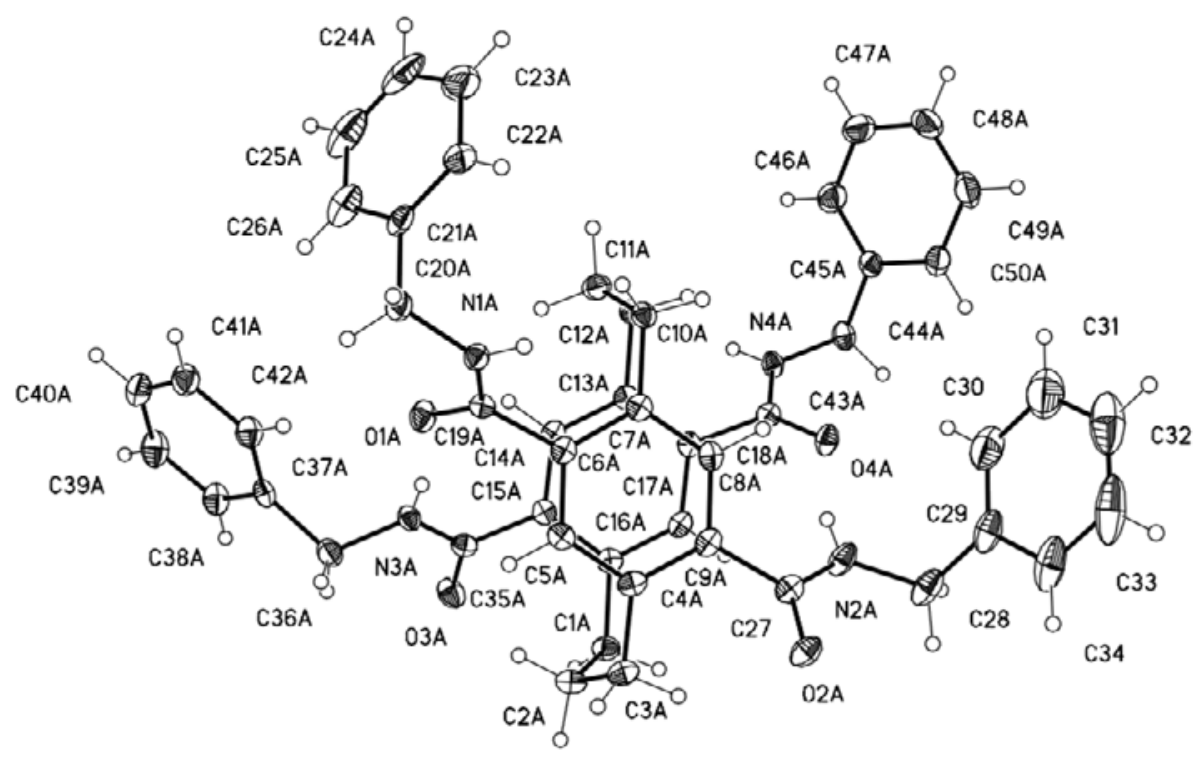

Figure S27. Thermal displacement ellipsoids showing $\left(\boldsymbol{S}_{p}\right)$-1c with a view from above the [3.3]paracyclophane axis. 


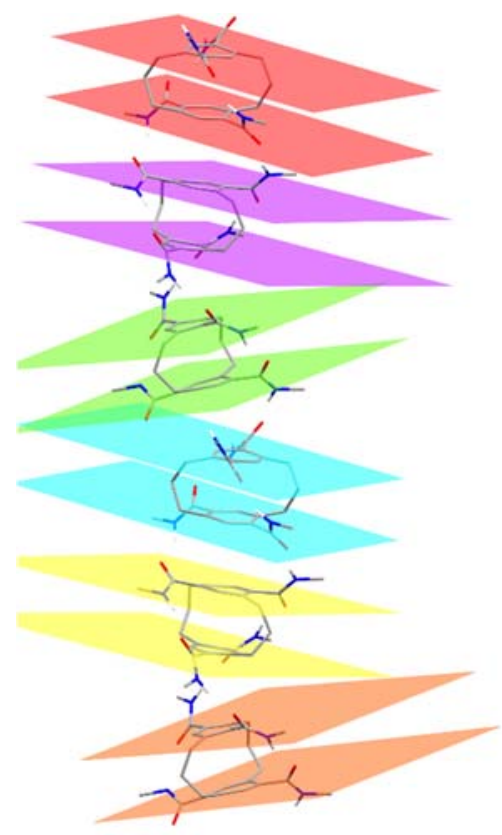

Figure S28. Asymmetric unit of $\left(\boldsymbol{S}_{p}\right)$-1c with planes drawn through each benzene deck, demonstrating "wobbling" along the stack. Benzyl side-chain omitted for clarity.

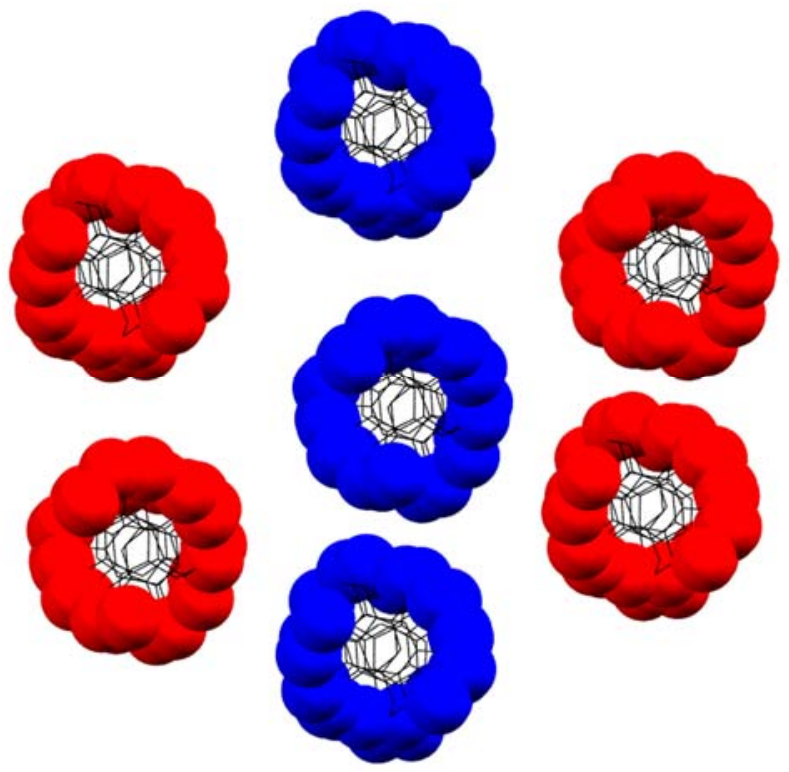

Figure S29. Packing of $\left(S_{p}\right)$-1c (red) and $\left(\boldsymbol{R}_{p}\right)$-1c (blue) in the solid state, viewed from above the propagation axis. 


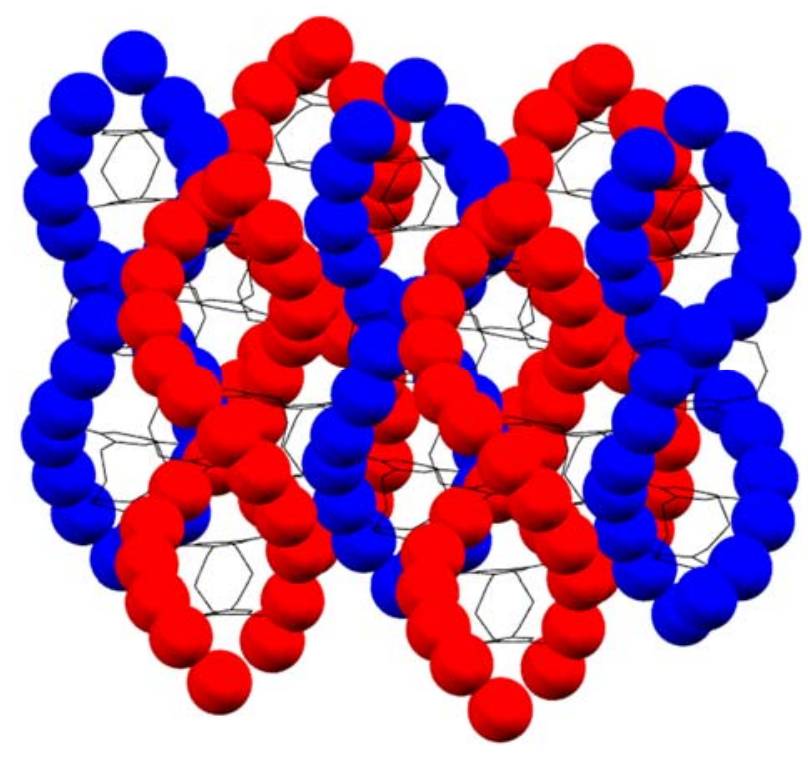

Figure S30. Packing of $\left(S_{p}\right) \mathbf{1 c}$ (red) and $\left(\boldsymbol{R}_{p}\right)$-1c (blue) in the solid state, viewed from the side of the propagation axis.

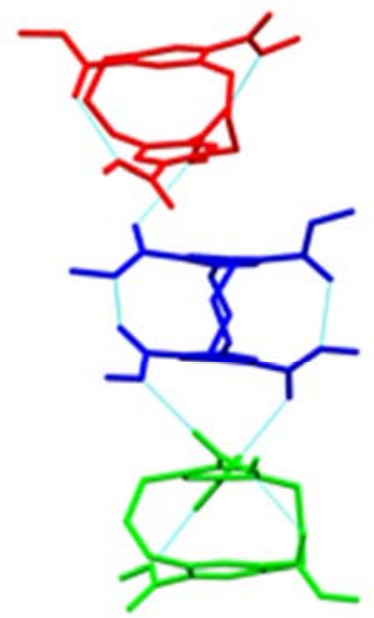

$\left(S_{\mathrm{p}}\right)_{3}$

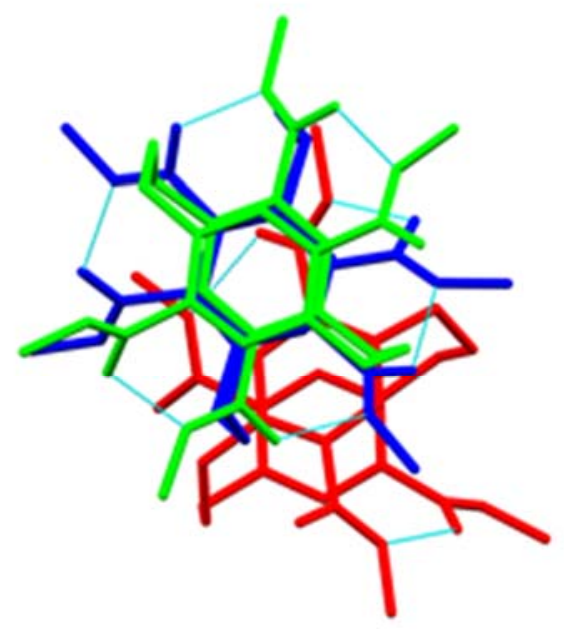

$\left(S_{\mathrm{p}}\right)_{3}$

Figure S31. Trimer of $\left(\boldsymbol{S}_{p}\right)$-1c with missing H-bond between red and blue monomers (left), resulting in a lack of cofacial overlap (right). 


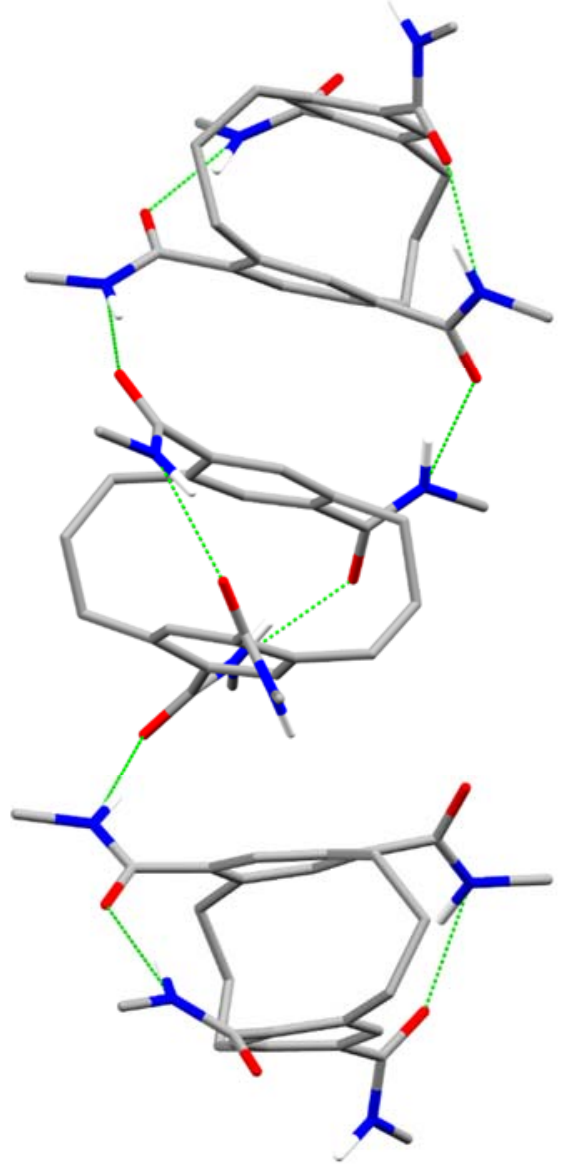

Figure S32. Trimer of $\left(\boldsymbol{S}_{p}\right)$-1c in the solid state highlighting hydrogen bonding (green). Bottom two monomers are participating in one intermolecular hydrogen bond. 


\section{INFRARED SPECTROSCOPY}

\section{General Information}

Solution phase IR spectra were collected under $\mathrm{N}_{2}$ atmosphere using a Bruker Vertex 80v with a sealed $1 \mathrm{~mm}$ pathlength $\mathrm{CaF}_{2}$ solution cell (PIKE Technologies). Solutions were prepared using HPLC grade chloroform and methylcyclohexane purchased from Sigma-Aldrich and dried over activated molecular sieves ( $4 \AA$ ) prior to use. Solid-state IR spectra were collected using using a Bruker Vertex 80v equipped with a GladiATR ${ }^{\mathrm{TM}}$ attachment (PIKE Technologies). Solid samples were dried under vacuum prior to measurement. All measurements were taken using Opus 7.0 software.

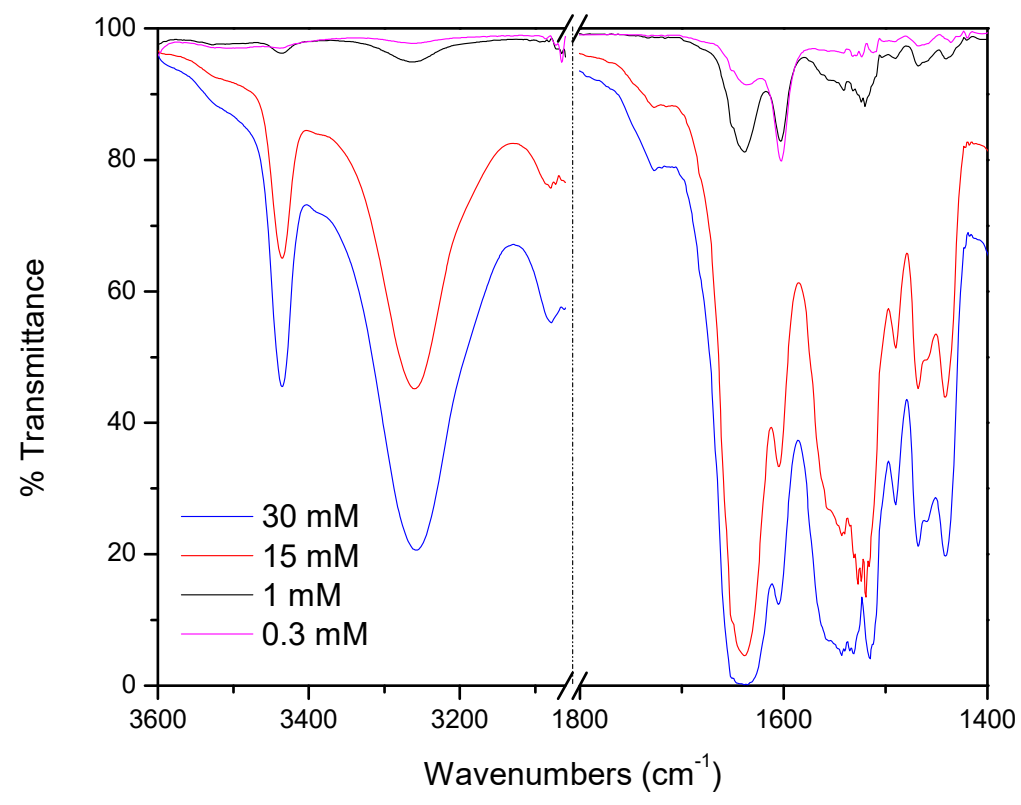

Figure S33. Overlaid FT-IR spectra of $( \pm)-\mathbf{1 a}\left(1-30 \times 10^{-3} \mathrm{M}\right)$ in $\mathrm{CHCl}_{3}$ at $298 \mathrm{~K}$. 


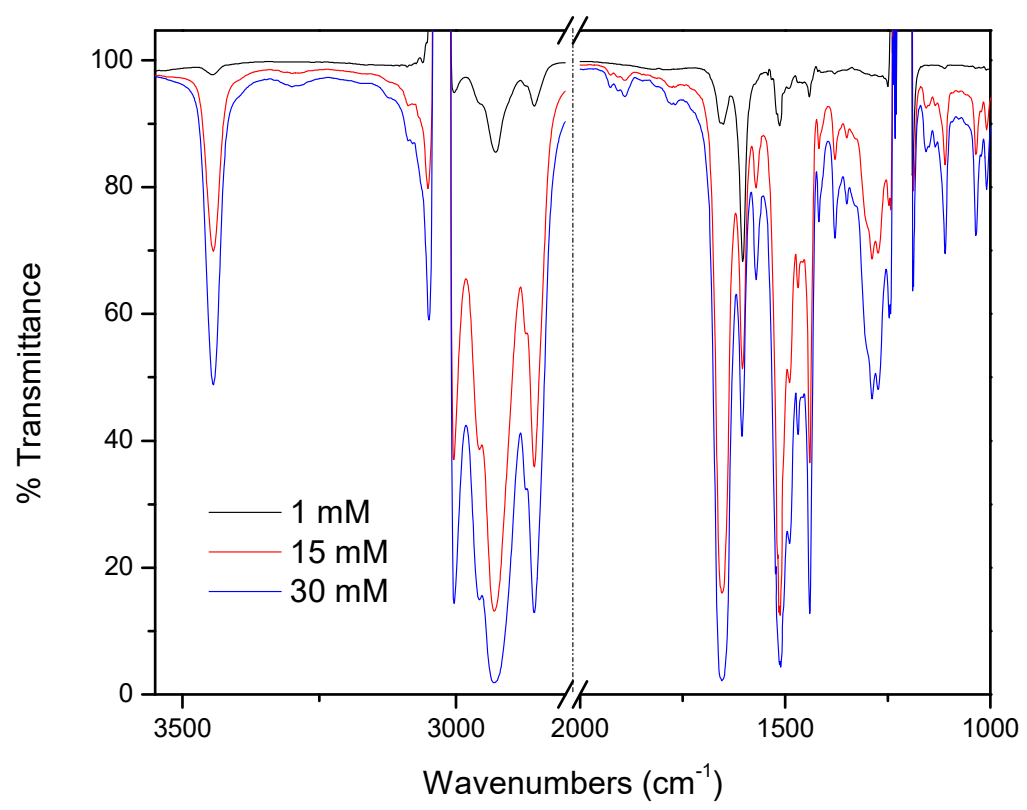

Figure S34. Overlaid FT-IR spectra of $( \pm)-3\left(1-30 \times 10^{-3} \mathrm{M}\right)$ in $\mathrm{CHCl}_{3}$ at $298 \mathrm{~K}$.

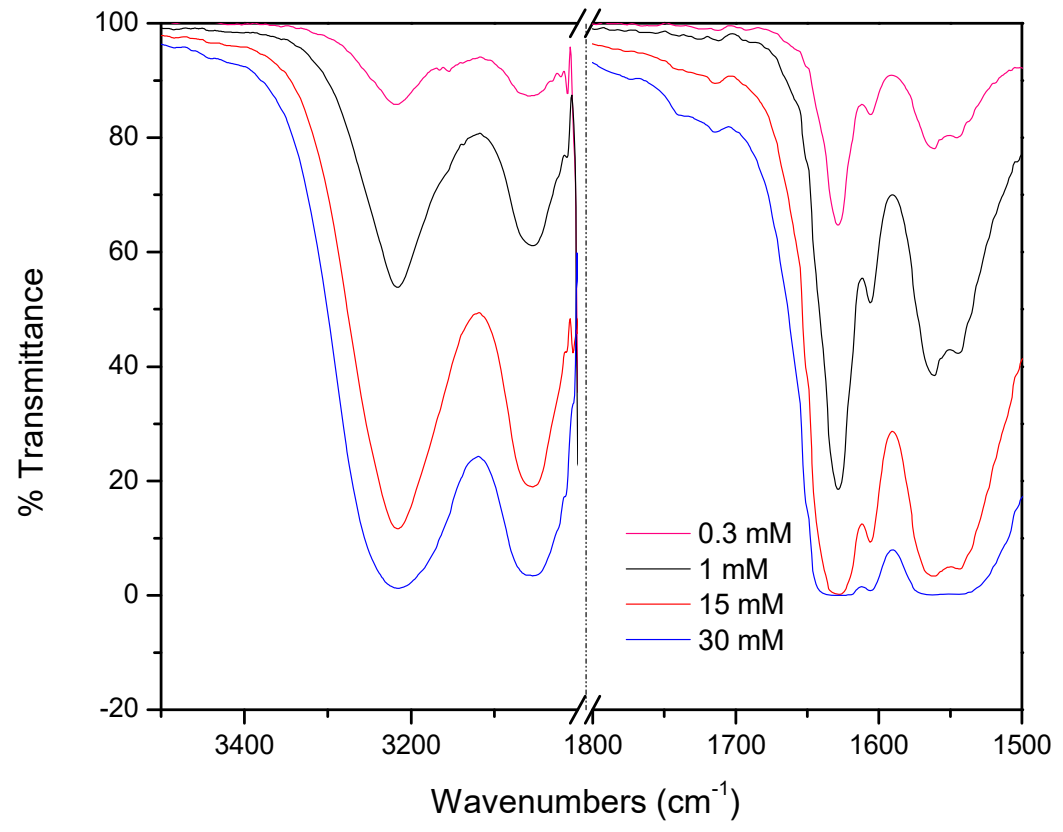

Figure S35. Overlaid FT-IR spectra of $( \pm)-\mathbf{1 a}\left(0.3-30 \times 10^{-3} \mathrm{M}\right)$ in methylcyclohexane at $298 \mathrm{~K}$. 


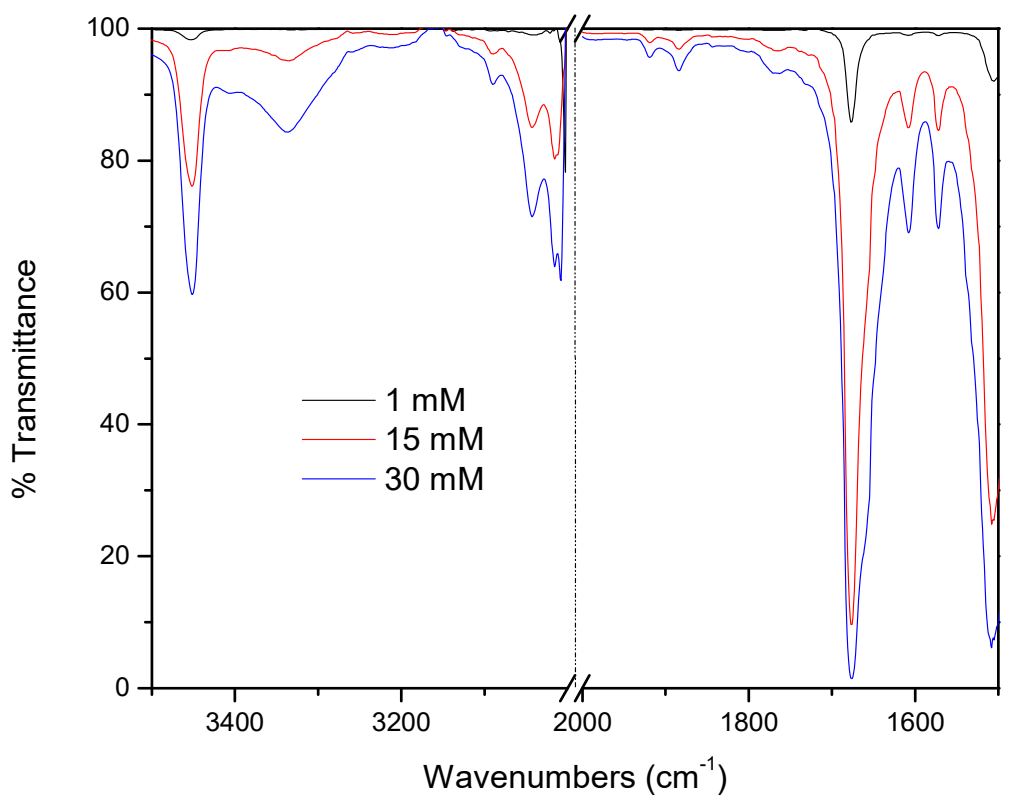

Figure S36. Overlaid FT-IR spectra of $( \pm)-3\left(1-30 \times 10^{-3} \mathrm{M}\right)$ inmethylcyclohexane at $298 \mathrm{~K}$.

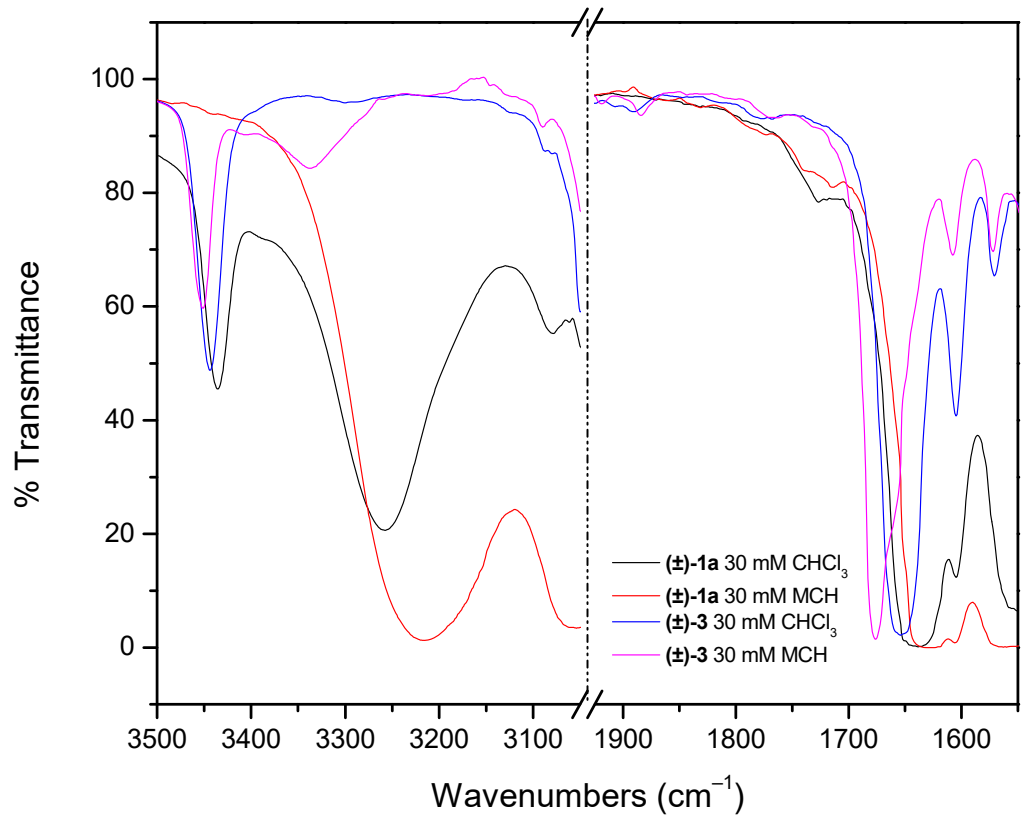

Figure S37. Overlaid FT-IR spectra of $( \pm)-1$ a and $( \pm)-3\left(30 \times 10^{-3} \mathrm{M}\right)$ in methylcyclohexane and chloroform at 298 K. 


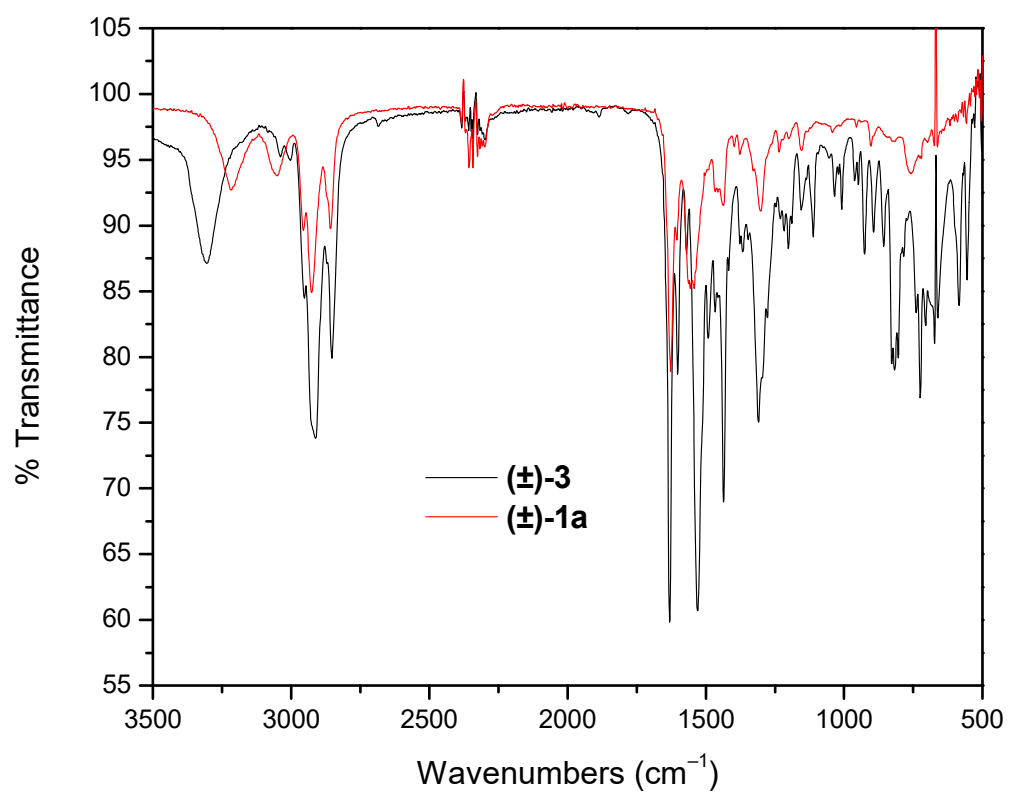

Figure S38. Overlaid FT-IR (ATR) spectra of $( \pm)-\mathbf{1 a}$ and $( \pm)-\mathbf{3}$ in the solid state. 
Table S4. Infrared frequencies of selected N-H stretches.

\begin{tabular}{|c|c|c|c|}
\hline Sample & Solvent & $\begin{array}{c}\mathrm{N}-\mathrm{H}\left(\mathrm{cm}^{-1}\right) \\
\text { (solvated) }\end{array}$ & $\begin{array}{c}\mathrm{N}-\mathrm{H}\left(\mathrm{cm}^{-1}\right) \\
\text { (hydrogen bonded) }\end{array}$ \\
\hline$( \pm)-1 a$ & $0.3 \mathrm{mM} \mathrm{CHCl}_{3}$ & 3436 & 3265 \\
\hline$( \pm)-1 \mathbf{a}$ & $1 \mathrm{mM} \mathrm{CHCl}_{3}$ & 3436 & 3265 \\
\hline$( \pm)-1 \mathbf{a}$ & $15 \mathrm{mM} \mathrm{CHCl}_{3}$ & 3436 & 3260 \\
\hline$( \pm)-\mathbf{1 a}$ & $30 \mathrm{mM} \mathrm{CHCl}_{3}$ & 3436 & 3257 \\
\hline$( \pm)-1 \mathbf{a}$ & $0.3 \mathrm{mM}$ methylcyclohexane & - & 3219 \\
\hline$( \pm)-1 \mathbf{a}$ & $1 \mathrm{mM}$ methylcyclohexane & - & 3219 \\
\hline$( \pm)-1 \mathbf{a}$ & 15 mM methylcyclohexane & - & 3219 \\
\hline$( \pm)-1 \mathbf{a}$ & $30 \mathrm{mM}$ methylcyclohexane & - & 3219 \\
\hline$( \pm)-1 \mathbf{a}$ & Solid State & - & 3219 \\
\hline$( \pm)-3$ & $1 \mathrm{mM} \mathrm{CHCl}_{3}$ & 3444 & - \\
\hline$( \pm)-3$ & $15 \mathrm{mM} \mathrm{CHCl}_{3}$ & 3444 & - \\
\hline$( \pm)-3$ & $30 \mathrm{mM} \mathrm{CHCl}_{3}$ & 3444 & - \\
\hline$( \pm)-3$ & $1 \mathrm{mM}$ methylcyclohexane & 3453 & - \\
\hline$( \pm)-3$ & $15 \mathrm{mM}$ methylcyclohexane & 3452 & 3336 \\
\hline$( \pm)-3$ & $30 \mathrm{mM}$ methylcyclohexane & 3451 & 3337 \\
\hline$( \pm)-3$ & Solid State & - & 3304 \\
\hline
\end{tabular}




\section{UV-VIS SPECTROSCOPY}

Absorbance measurements were obtained on a Cary 100 Bio UV-Visible dual beam spectrophotometer controlled by Cary Win UV software and equipped with a Peltier $1 \times 1$ Cell Holder using capped $1 \mathrm{~cm}$ quartz cells. Spectrophotometric grade methylcyclohexane was purchased from Sigma-Aldrich. Temperature-dependent UV data in methylcyclohexane was measured using a constant cooling ramp of either $1 \mathrm{~K}$ per minute or $1.4 \mathrm{~K}$ per minute.

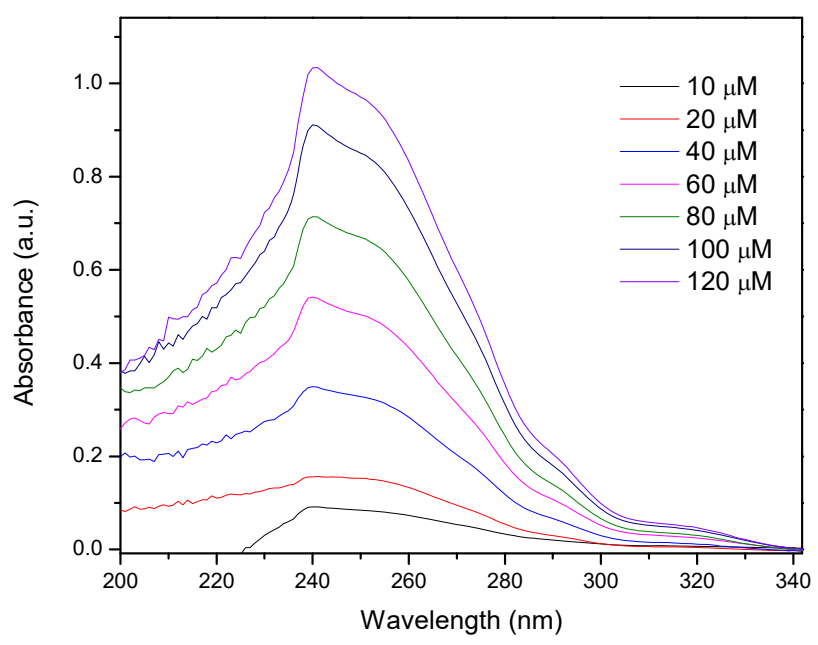

Figure S39. Overlaid UV-vis spectra of $( \pm)-1 \mathrm{a}\left(1-12 \times 10^{-5} \mathrm{M}\right)$ in chloroform at $298 \mathrm{~K}$.

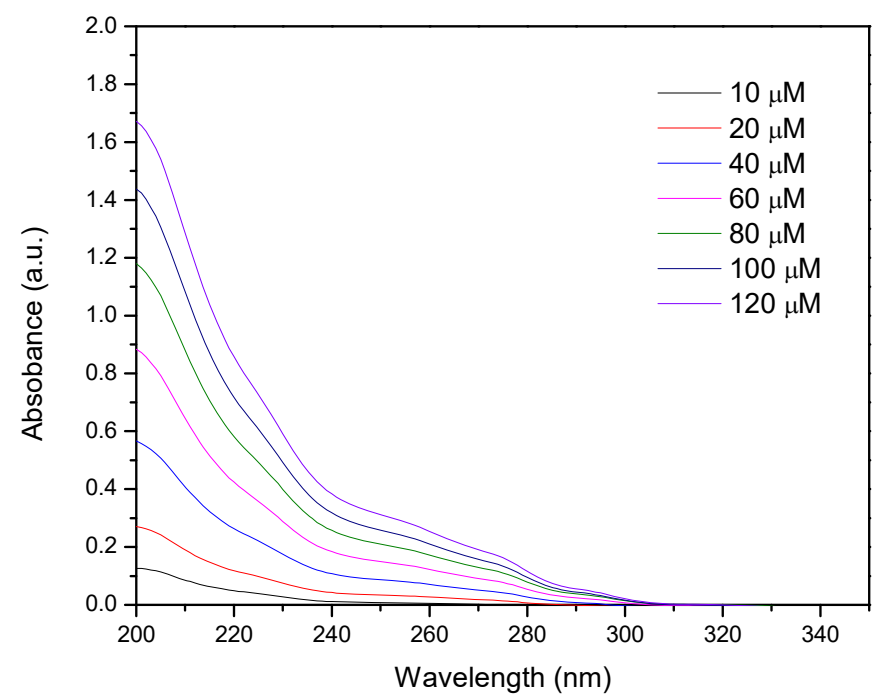

Figure S40. Overlaid UV-vis spectra of $( \pm)-1 a\left(1-12 \times 10^{-5} \mathrm{M}\right)$ in methylcyclohexane at $298 \mathrm{~K}$. 


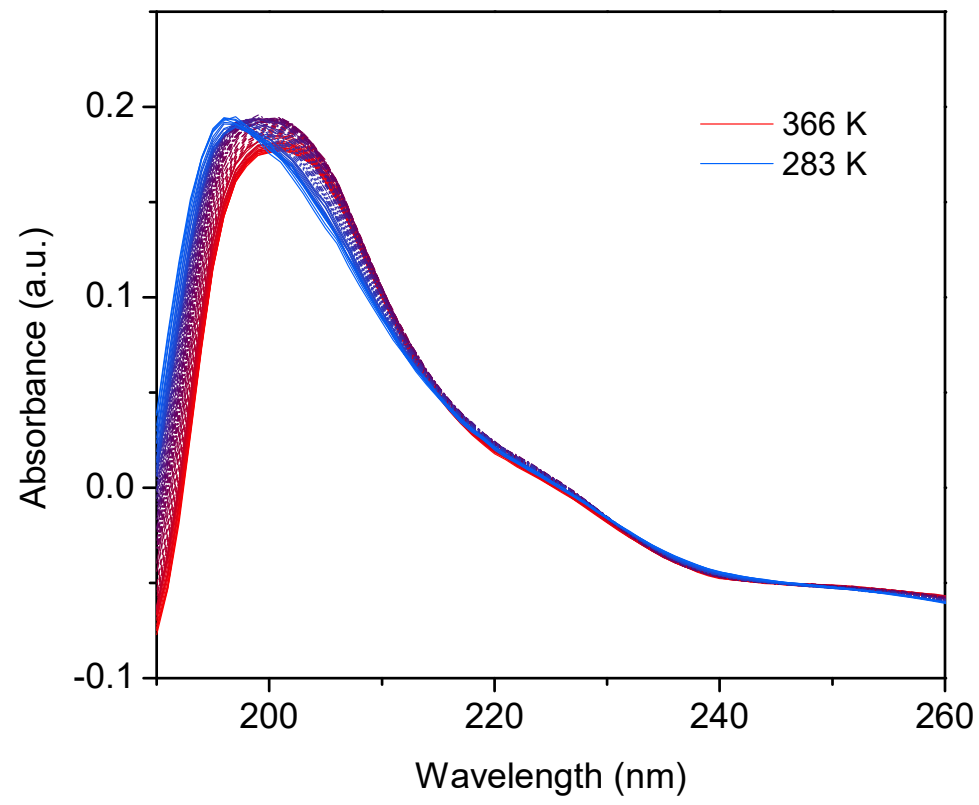

Figure S41. Density corrected UV-vis spectra of $( \pm)-1 \mathrm{a}\left(1.2 \times 10^{-4} \mathrm{M}\right)$ from $366-283 \mathrm{~K}$, every $1 \mathrm{~K}$ at a cooling rate of $1 \mathrm{~K} \mathrm{~min}^{-1}$ in methylcyclohexane.

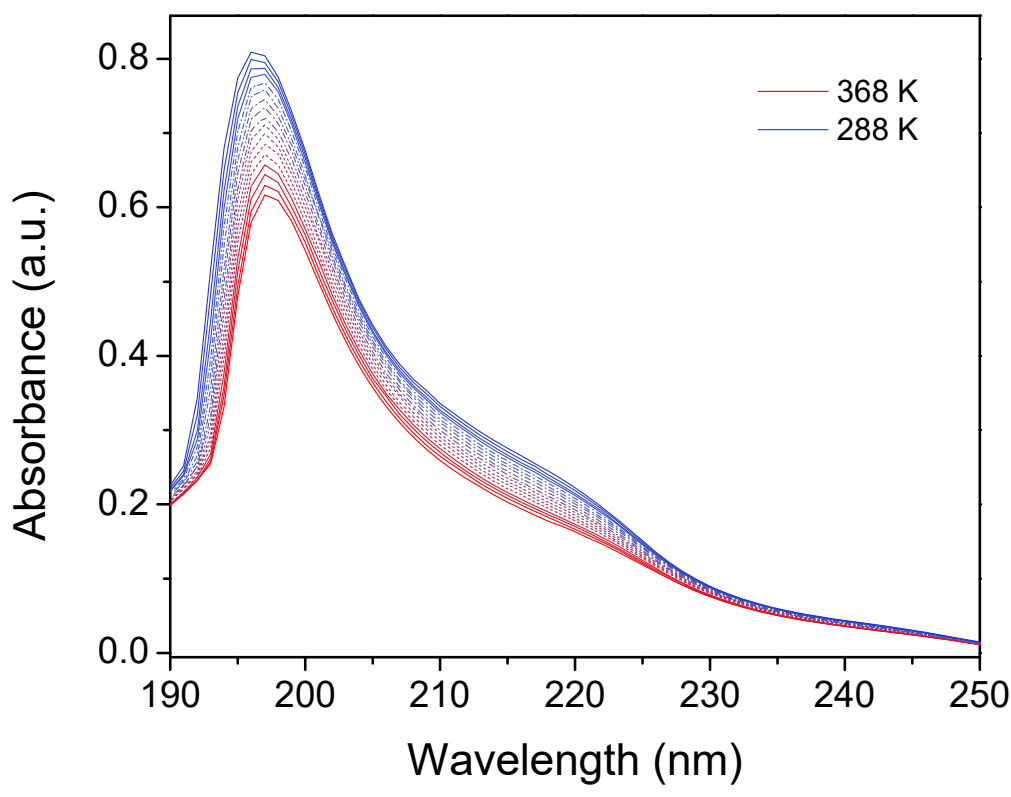

Figure S42. Density corrected UV-vis spectra of $( \pm)-3\left(4.0 \times 10^{-5} \mathrm{M}\right)$ from $368-288 \mathrm{~K}$, every $5 \mathrm{~K}$ at a cooling rate of $1.4 \mathrm{~K} \mathrm{~min}^{-1}$ in methylcyclohexane. 


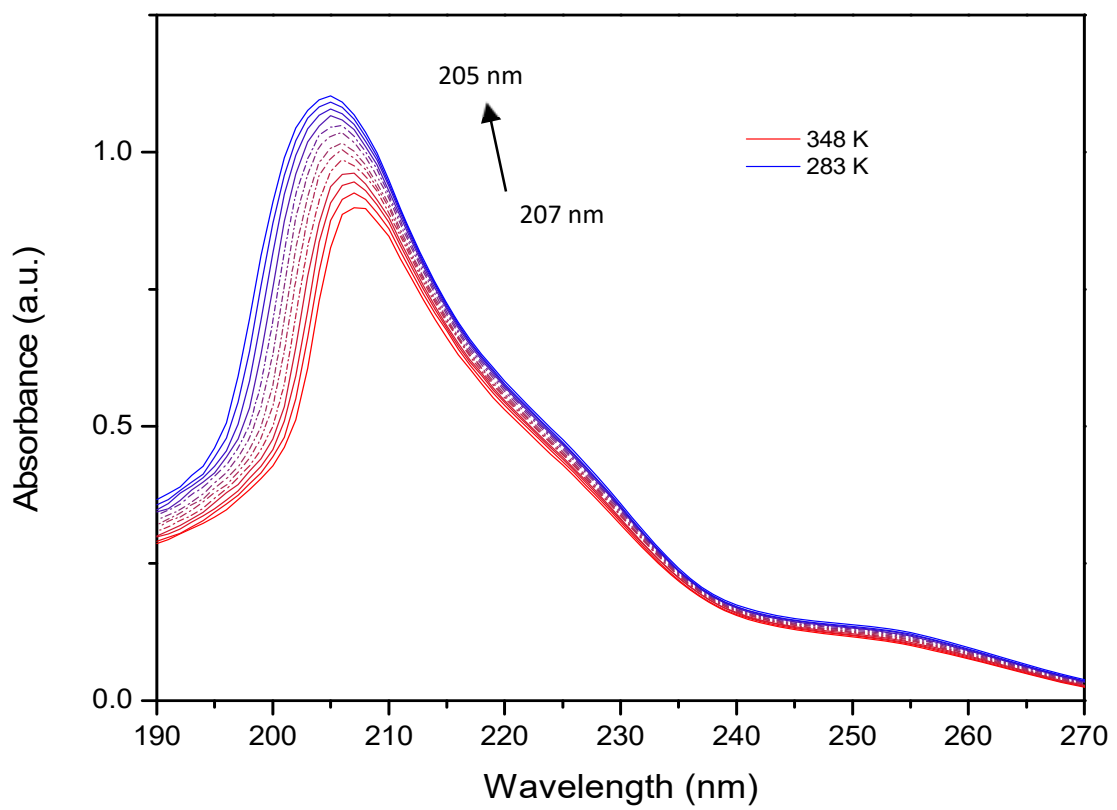

Figure S43. Density corrected UV-vis spectra of $( \pm)-1 \mathbf{a}\left(2.0 \times 10^{-5} \mathrm{M}\right)$ from $348-283 \mathrm{~K}$, every $5 \mathrm{~K}$ at a cooling rate of $1.4 \mathrm{~K} \mathrm{~min}^{-1}$ in ethanol.

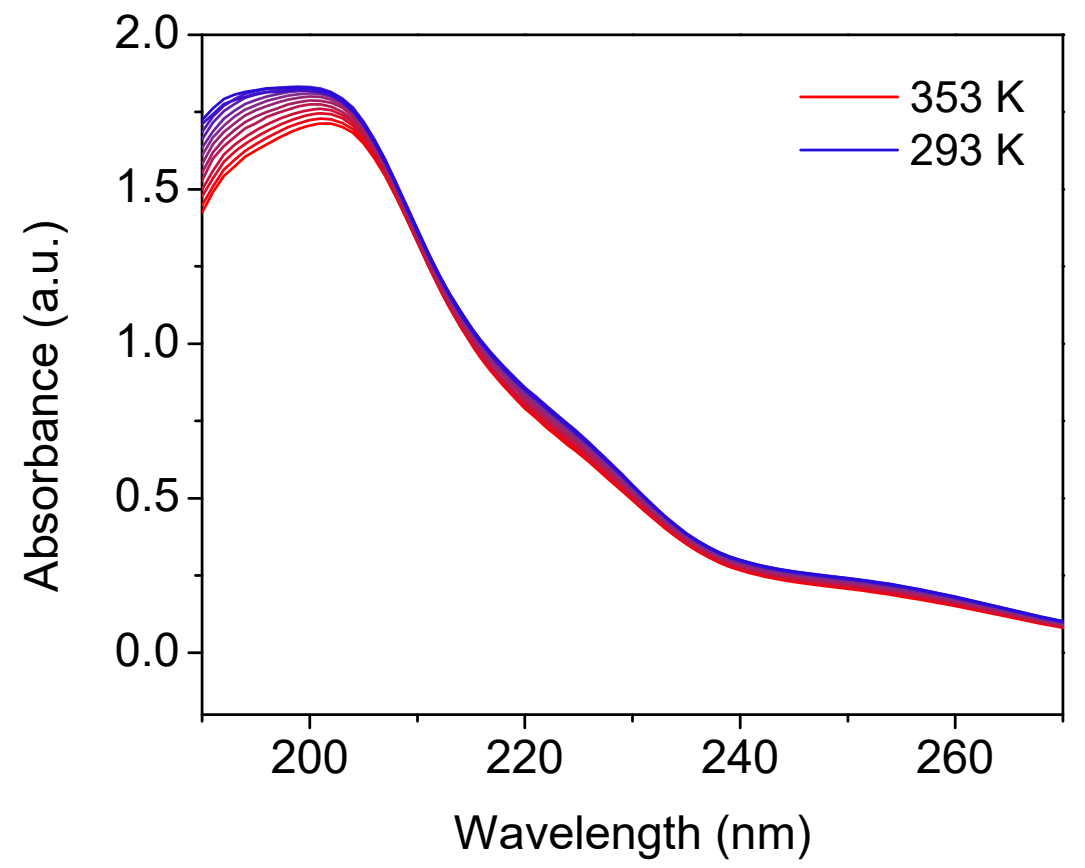

Figure S44. Variable temperature UV-Vis spectra of $( \pm)-3\left(2.0 \times 10^{-5} \mathrm{M}\right)$ from $353-293 \mathrm{~K}$, every $5 \mathrm{~K}$ at a cooling rate of $1.4 \mathrm{~K} \mathrm{~min}^{-1}$ in acetonitrile. 


\section{REFERENCES}

1. Matsuda-Sentou, W.; Shinmyozu, T., A Synthetic and Structural Study of 17,18-

Dicyano[32] $(1,6)$ cyclooctatetraen $(1,4)$ cyclophane Generated by Photolysis of [32](1,4)Barrelenophane. European Journal of Organic Chemistry 2000, 2000 (18), 3195-3203.

2. Sasaki, H.; Kitagawa, T., Synthesis of [3n]Cyclophanes and Related Compounds by Alkylation of Tosylmethyl Isocyanide with Bis (bromomethyl) benzenes. Chemical \& Pharmaceutical Bulletin 1983, 31 (8), 2868-2878.

3. Shibahara, M.; Watanabe, M.; Iwanaga, T.; Matsumoto, T.; Ideta, K.; Shinmyozu, T., Synthesis, Structure, and Transannular $\pi-\pi$ Interaction of Three- and Four-Layered [3.3]Paracyclophanes. The Journal of Organic Chemistry 2008, 73 (12), 4433-4442.

4. Fagnani, D. E.; Meese, M. J., Jr.; Abboud, K. A.; Castellano, R. K., Homochiral

[2.2]Paracyclophane Self-Assembly Promoted by Transannular Hydrogen Bonding. Angewandte Chemie International Edition 2016, 55 (36), 10726-10731.

5. Shibahara, M.; Watanabe, M.; Miyazaki, T.; Goto, K.; Matsumoto, T.; Shinmyozu, T., Synthesis of Dibromo[3.3]paracyclophanes. Synthesis 2016, 48 (08), 1197-1201.

6. Martin, R. B., Comparisons of Indefinite Self-Association Models. Chemical Reviews 1996, 96 (8), 3043-3064.

7. Seo, M.; Kim, J. H.; Kim, J.; Park, N.; Park, J.; Kim, S. Y., Self-Association of Bis-Dendritic Organogelators: The Effect of Dendritic Architecture on Multivalent Cooperative Interactions. Chemistry - A European Journal 2010, 16 (8), 2427-2441.

8. Smulders, M. M. J.; Nieuwenhuizen, M. M. L.; de Greef, T. F. A.; van der Schoot, P.; Schenning, A. P. H. J.; Meijer, E. W., How to Distinguish Isodesmic from Cooperative Supramolecular Polymerisation. Chemistry - A European Journal 2010, 16 (1), 362-367.

9. CCDC $1949727[( \pm)-1 c]$ contains the supplementary crystallographic data for this paper. These data can be obtained free of charge from the Cambridge Crystallographic Data Centre. 